\title{
MODELAGEM DE CIRCUITOS NEURAIS DO SISTEMA NEUROMOTOR E PROPRIOCEPTOR DE INSETOS COM O USO DE TRANSFERÊNCIA DE INFORMAÇÃO ENTRE CONEXÕES NEURAIS
}

\author{
Wagner Endo \\ Tese apresentada à Escola de Engenha- \\ ria de São Carlos da Universidade de São \\ Paulo, como parte dos requisitos para ob- \\ tenção do título de Doutor em em Ciên- \\ cias, Programa de Engenharia Elétrica
}

ORIENTADOR: Prof. Dr. Carlos Dias Maciel ÁREA DE CONCENTRAÇÃO: Sistemas Dinâmicos

Trata-se da versão corrigida da tese. A versão original se encontra disponível na EESC/USP que aloja o Programa de Pós-Graduação de Engenharia Elétrica. 
AUTORIZO A REPRODUC̄̃O TOTAL OU PARCIAL DESTE TRABALHO, POR QUALQUER MEIO CONVENCIONAL OU ELETROONICO, PARA FINS DE ESTUDO E PESQUISA, DESDE QUE CITADA A FONTE.

Endo, Wagner

Modelagem de Circuitos Neurais do Sistema
Neuromotor e Proprioceptor de Insetos com o uso da

Transferência de Informação entre conexões neurais /

Wagner Endo; orientador Carlos Dias Maciel. São Carlos, 2014.

Tese (Doutorado) - Programa de Pós-Graduação em Engenharia Elétrica e Área de Concentração em Sistemas Dinâmicos -- Escola de Engenharia de São Carlos da Universidade de São Paulo, 2014.

1. neurociência motora. 2. conectividade neural. 3. engenharia bioinspirada. 4. STA. 5. transferência de informação. 6. transferência de entropia. 7. nível de significância estatística. I. Título. 


\section{FOLHA DE JULGAMENTO}

Candidato: Engenheiro WAGNER ENDO.

Título da tese: "Modelagem de circuitos neurais do sistema neuromotor e proprioceptor de insetos com o uso de transferência de informação entre conexões neurais".

Data da defesa: $31 / 03 / 2013$

\section{Comissão Julgadora:}

Prof. Associado Carlos Dias Maciel (Orientador)

(Escola de Engenharia de São Carlos/EESC)

Prof. Associado Marco Henrique Terra

(Escola de Engenharia de São Carlos/EESC)

Prof. Dr. Carlos Henrique Costa Ribeiro

(Instituto Tecnológico de Aeronáutica/ITA)

Prof. Dr. Ailton Akira Shinoda

(Universidade Estadual Paulista "Júlio de Mesquita Filho"/UNESP-Ilha Solteira)

Prof. Dr. Eugênio Eduardo de Oliveira (Universidade Federal de Viçosa/UFV)
Resultado:
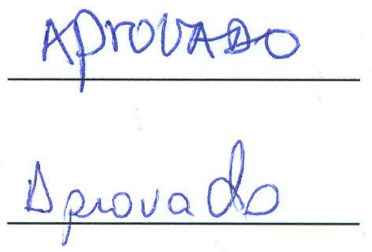

APROVADO

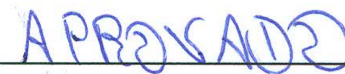
A.PROVADO

Coordenador do Programa de Pós-Graduação em Engenharia Elétrica e Presidente da Comissão de Pós-Graduação:

Prof. Titular Denis Vinicius Coury 
Dedicatória

Dedico este trabalho à minha esposa, Thaís, e aos meus pais e irmãos. 
Em primeiro lugar agradeço a Deus, não especificamente ao que nos referimos religiosamente, mas àquela fonte de vida, de fé e de força que parece ser uma resposta sem fim.

Agradeço ao meu orientador, professor Carlos Dias Maciel, por me oportunizar este trabalho de pesquisa e mais ainda pelos desafios colocados a mim. Pois, foram esses que me fizeram superar limites.

Um agradecimento a minha esposa Thaís, pois ela foi minha grande companheira desta batalha, a pessoa que esteve do meu lado, mesmo sem eu poder lhe dar toda a atenção que merecia.

Agradeço à Universidade Tecnológica Federal do Paraná (UTFPR) por ter viabilizado o afastamento para que eu pudesse finalizar este trabalho.

Também, agradeço aos meu pais, pois são deles minha base e referência de vida. Aos meus irmãos, Evandro e Viviane, pela força que sempre me deram.

Agradeço ao Professor Phillip L. Newland, da Universidade de Southampton, UK. Pois, todas as suas contribuições, sugestões e orientações foram essenciais para a conclusão deste trabalho.

Agradeço aos membros da minha banca de qualificação, professores Eugênio E. de Oliveira e Carlos H. C. Ribeiro, pela sugestões para melhorar este trabalho. Ainda, reforço o agradecimento ao professor Eugênio, pela acolhida em seu Laboratório de Entomologia na Universidade Federal de Viçosa.

Obrigado aos companheiros do LPS, pelas conversas, trocas de ideia e apoio.

Por fim, um agradecimento especial aos companheiros e amigos da UTFPR, Campus Cornélio Procópio, que sempre acreditaram em mim. E a quem devo minha eterna gratidão pelos conselhos, pela força, pelo apoio e, principalmente, por eu sempre poder contar com suas amizades. 
"O acaso (aleatório) vai me proteger, enquanto eu andar distraído.”

Titãs

“(...) a resposta humana à incerteza é tão complexa que, por vezes, distintas estruturas cerebrais chegam a conclusões diferentes para determinar qual delas dominará as demais - as crenças e a intuição muitas vezes nos deixam em maus lençóis.” Leonard Mlowdinow 


\section{Sumário}

Lista de Figuras $\quad$ xv

Lista de Tabelas

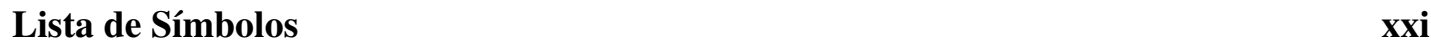

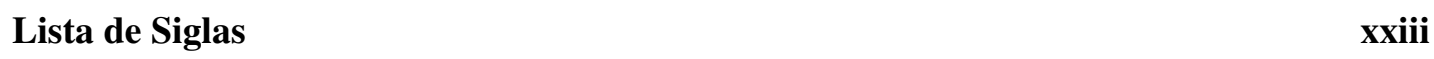

$\begin{array}{lll}\text { Resumo } & \text { Xxv }\end{array}$

$\begin{array}{lll}\text { Abstract } & \text { xxix }\end{array}$

1 Introdução 1

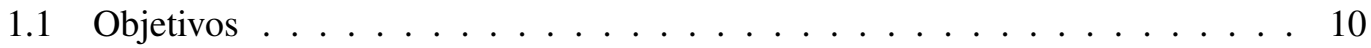

$1.1 .1 \quad$ Objetivos específicos . . . . . . . . . . . . . 10

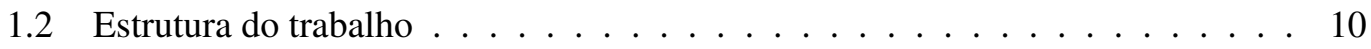

2 Sistema neuromotor de um inseto $\quad 13$

2.1 Uma breve introdução à neurofisiologia . . . . . . . . . . . . . . . . . . . . 13

2.2 Processamento e controle local dos membros locomotores . . . . . . . . . . . 16

2.2.1 Estudo comparado de controle e processamento do sistema proprioceptor 16

2.2.2 Conexões sinápticas relacionadas ao processamento local . . . . . . . 18

2.3 Neurofisiologia e estrutura do sistema nervoso central e motor de gafanhotos . 20

2.3 .1 Gânglios torácicos . . . . . . . . . . . . . . . . . . 23

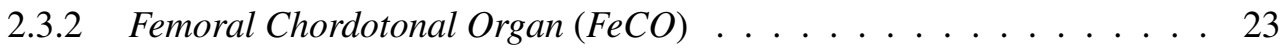

2.4 Caracterização dos neurônios e tipos de sinais neurais do inseto . . . . . . . . 24

2.4 .1 Neurônios sensores . . . . . . . . . . . . . . . . . . . . . 25

2.4 .2 Neurônios motores . . . . . . . . . . . . . . . . . . . . . . 26

2.4.3 Interneurônios locais: spiking e nonspiking . . . . . . . . . . . . . 27

3 Estudos de medidas de associação: transferência de entropia e informação. 33 
3.1 Medidas de associação de primeira ordem entre séries temporais . . . . . . . . 33

3.1.1 Estudo das curvas médias de excitação para sinais neurais spiking Análise do Spike Triggered Average . . . . . . . . . . . . . . . . . . . 34

3.1 .2 Correlação cruzada . . . . . . . . . . . . . . . . . . . . . . . . 36

3.2 Entropia e informação mútua como medidas de associação . . . . . . . . . . . 37

3.3 Transferência de informação . . . . . . . . . . . . . . . . . . . . . . . . . 39

3.3.1 Atraso da informação mútua . . . . . . . . . . . . . . . . . . . . 40

3.3.2 Transferência de entropia . . . . . . . . . . . . . . . . 42

3.4 Utililização de Surrogate Data e medidas do nível de significância . . . . . . . 44

3.5 Normalização das medidas de transferência de informação . . . . . . . . . . . . 45

4 Materiais e métodos 49

4.1 Algoritmos . . . . . . . . . . . . . . . . . . 51

4.1.1 Procedimentos para obtenção da curva média de excitação dos sinais

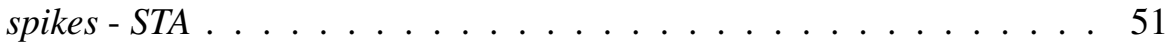

4.1.2 Estimativa das funções densidade de probabilidades . . . . . . . . . 52

4.1.3 Estimativa da informação mútua atrasada e da transferência de entropia 53

4.1.4 Determinação do Surrogate Data . . . . . . . . . . . . . . . . . 57

4.2 Descrição e modelos dos dados . . . . . . . . . . . . . . . . . 60

4.2 .1 Dados simulados . . . . . . . . . . . . . . . . . . . . . . 60

4.2 .2 Dados reais . . . . . . . . . . . . . . . . . . . . . . . . 61

4.3 Modelo da estrutura de múltiplos padrões de caminhos da transferência de informação no sistema neuromotor . . . . . . . . . . . . . . . . . . . . 68

$5 \quad$ Resultados e discussões $\quad 73$

5.1 Resultados das análises do STA para neurônios sensores . . . . . . . . . . . 74

5.2 Resultados dos dados de simulação para a transferência de informação . . . . . 78

5.3 Resultados dos dados reais para análise de conectividade do sistema neuromotor de insetos . . . . . . . . . . . . . . . . . 81

5.3 .1 Neurônios sensores . . . . . . . . . . . . . . . . . 81

5.3.2 Interneurônios spiking . . . . . . . . . . . . . . . . . 87

5.3.3 Interneurônios nonspiking ～. . . . . . . . . . . . . . . . . 90

5.4 Mapeamento da transferência de informação e o modelo estrutural do circuito neural do sistema neuromotor . . . . . . . . . . . . . . . . . . . 95

6 Publicações e Submissões 101

$\begin{array}{lll}7 & \text { Considerações finais e próximas etapas } & 103\end{array}$ 
Referências Bibliográficas 


\section{Lista de Figuras}

FIGURA 1.1 Exemplo da imagem de um conectoma do cerébro humano . . . . . . 3

FIGURA 1.2 Modelo de conectividade com histórico comum de informação . . . . 6

FIGURA 1.3 Diagrama de Venn da informação mútua e transferência de entropia . 7

FIGURA 1.4 Exemplo de análise da transferência de entropia . . . . . . . . . . . 8

FIGURA 2.1 Representação de uma conexão sináptica $\ldots \ldots$. . . . . . . . . . . . 14

FIGURA 2.2 Exemplo de um circuito neural com típicas redes de conexões sinápticas 15

FIGURA 2.3 Diagrama de geração da força muscular . . . . . . . . . . . . . . 16

FIGURA 2.4 Circuitos neurais sensores para interfaces cérebro-computador. . . . . 17

FIGURA 2.5 Circuito neural do reflexo local da perna . . . . . . . . . . . 20

FIGURA 2.6 Desenho de um gafanhoto do deserto Schistocerca Gregaria e o diagrama de seu sistema nervoso . . . . . . . . . . . . . . . . . . . . 21

FIGURA 2.7 Representação do gânglio torácico de um inseto . . . . . . . . . . . . 23

FIGURA 2.8 Ganglio metatorácico do sistema neuromotor da membro posterior

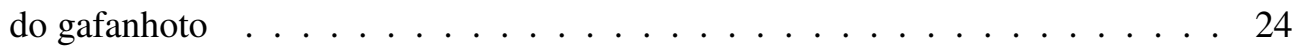

FIGURA 2.9 Localização e estrutura do $\mathrm{Fe} C O$. . . . . . . . . . . . . . . . . . 25

FIGURA 2.10 Diagrama de convergência neural no gânglio metatorácico para os interneurônios anteromediais . . . . . . . . . . . . . . . . . . . . . . . 29

FIGURA 2.11 Diagrama de convergência neural do gânglio metatorácico para os interneurônios midline spiking . . . . . . . . . . . . . . . . . . 30 
FIGURA 3.1 Esquemático com o procedimento para cálculo do STA . . . . . . 35

FIGURA 3.2 Relação de entropia entre duas variáveis aleatórias . . . . . . . . . . 38

FIGURA 3.3 Modelo da transferência de informação entre séries temporais . . . . 41

FIGURA 3.4 Padrões de relações causais $\ldots \ldots \ldots$. . . . . . . . . . . . 42

FIGURA 4.1 Modelo geral para o método de estímulo e coleta intracelular de sinais do sistema neural do inseto f . . . . . . . . . . . . . . . . 49

FIGURA 4.2 Modelo para obtenção do spike-triggered average . . . . . . . . . . . 52

FIGURA 4.3 Densidades de probabilidades para a informação mútua . . . . . . . . 54

FIGURA 4.4 Modelo de análise das séries temporais para a transferência de entropia 57

FIGURA 4.5 Procedimentos para geração do Surrogate Data e estimativas dos níveis de significância . . . . . . . . . . . . . . . . . . . . . . 58

FIGURA 4.6 Diagrama de blocos da geração dos sinais eletromecânicos . . . . . . 62

FIGURA 4.7 Esquema experimental para excitação dos neurônios motores através do apodeme . . . . . . . . . . . . . . . . . 63

FIGURA 4.8 Amostra de 1 segundo da coleta de dados do experimento com gafanhotos . . . . . . . . . . . . . . . . . . . . . . . 64

FIGURA 4.9 Grupo de sinais gravados nos arquivos do banco de dados . . . . . 65

FIGURA 4.10 Determinação dos trechos estacionários pela capacidade do canal 66

FIGURA 4.11 Pico de transição no início do estímulo neural . . . . . . . . . . . . . 67

FIGURA 4.12 Circuito neural de resposta local entre o $\mathrm{FeCO}$ e os interneurônios . . 68

FIGURA 4.13 Modelos do circuito neural e dos sinais interneuronais. . . . . . . . . 70

FIGURA 5.1 Resultados das curvas médias dadas pelo análise de STA . . . . . . . 75

FIGURA 5.2 Funções densidade de probabilidades dos sinais de excitação e spikes do $\mathrm{FeCO}$ vindas da análise de $S T A \ldots \ldots \ldots$. . . . . . . . . . . 76

FIGURA 5.3 Comparação entre o STA e a correlação cruzada no modelo de neurônio sensor sensível ao movimento. . . . . . . . . . . . . . . . . . . . 77 
FIGURA 5.4 Comparação entre o STA e a correlação cruzada no modelo de neurônio sensor sensível à posição e aplicação de força. . . . . . . . . . . . . . . 77

FIGURA 5.5 Análise com dados sintéticos de simulação . . . . . . . . . . . . . 78

FIGURA 5.6 Análise dos dados simulados para a $T E$ e a $D M I \quad \ldots \ldots$. . . . . . . 80

FIGURA 5.7 Padrão de resultado da curva de informação mútua atrasada para

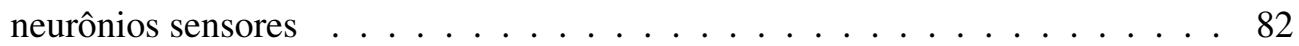

FIGURA 5.8 Curvas de transferência de informação pela DMI . . . . . . . . . . . 83

FIGURA 5.9 Transferência de entropia para os neurônios sensores . . . . . . . . 84

FIGURA 5.10 Resultados de diferentes padrões de atraso da transferência de informação nos neurônios sensores $\ldots \ldots$. . . . . . . . . . . . . 86

FIGURA 5.11 Respostas-padrão da informação mútua atrasada para sinais spiking 87

FIGURA 5.12 Distribuição dos tempos de atraso e suas respectivas informações mútuas para os interneurônios spiking . . . . . . . . . . . . 88

FIGURA 5.13 Resultados dos padrões de tempos para interneurônios spiking. . . . . 89

FIGURA 5.14 Clustering para os interneurônios spiking . . . . . . . . . . . . 90

FIGURA 5.15 Clustering dos padrões de tempos para interneurônios spiking. . . . . 91

FIGURA 5.16 Respostas-padrão da informação mútua atrasada para sinais nonspiking 91

FIGURA 5.17 Resultados dos padrões de tempos para interneurônios nonspiking. . . 92

FIGURA 5.18 Clustering para interneurônios nonspiking . . . . . . . . . . . . . 93

FIGURA 5.19 Clustering dos padrões de tempos para interneurônios nonspiking. . 94

FIGURA 5.20 Esquemático do circuito neural do sistema neuromotor do gafanhoto . 95

FIGURA 5.21 Grafo de conexões do gânglio metatorácico do sistema neuromotor do gafanhoto . . . . . . . . . . . . . . . . . . . . . . . 97

FIGURA 5.22 Polaridades da conectividade neural . . . . . . . . . . . . . . . . . 99

FIGURA 7.1 Grafo para informação mútua condicionada . . . . . . . . . . . . 107

FIGURA 7.2 Representação de um sistema de informação mútua condicionada . 108 


\section{Lista de Tabelas}

TABELA 2.1 Principais neurônios motores utilizados nas coletas de sinais . . . . . 27

TABELA 5.1 Valores máximos para a DMI para os dados simulados . . . . . . . 79

TABELA 5.2 Valores médios das máximas informações mútuas para os neurônios

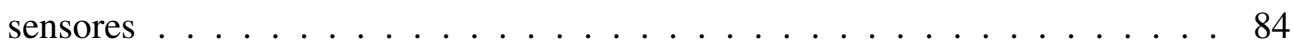

TABELA 5.3 Valores dos picos centrais baseados no valor médio dos atrasos e da máxima informação mútua atrasada do neurônios do $\mathrm{FeCO}$. . . . . . . . . . . 85

TABELA 5.4 Valores dos picos duplos baseados no valor médio dos atrasos e da máxima informação mútua atrasada do neurônios do $\mathrm{FeCO}$. . . . . . . . . . 86

TABELA 5.5 Valores médios das máximas informações mútuas para os interneurônios nonspiking . . . . . . . . . . . . . . . . . . . . . . . 94

TABELA 5.6 Valores da máxima informação mútua . . . . . . . . . . . . . . 96

TABELA 5.7 Valores dos tempos de atraso $\ldots \ldots \ldots$. . . . . . . . . . 96 


\section{Lista de Símbolos}

\begin{tabular}{|c|c|}
\hline$H(X)$ & entropia de $X$ \\
\hline$X$ & variável aleatória \\
\hline$p(x)$ & função de densidade de probabilidade da variável aleatória $X$ \\
\hline$\phi$ & conjunto de símbolos discretizados da codificação da variável aleatória $X$ \\
\hline$t$ & tempo $\ldots \ldots \ldots \ldots$ \\
\hline$\tau$ & tempo de atraso da transferência de informação ........ \\
\hline$k$ & índice do bloco de uma variável aleatória \\
\hline$X_{k}$ & bloco $k$ da variável aleatória $X \ldots$ \\
\hline$X_{k}^{\tau}$ & bloco $k$ da variável aleatória $X$ com um atraso $\tau$. \\
\hline$I(X ; Y)$ & informação mútua entre $X$ e $Y$. \\
\hline$\zeta_{X Y}$ & acoplamento (associação da informação) entre $X$ e $Y$. \\
\hline$\kappa$ & quantidade de dados ou número de observações \\
\hline$\eta$ & número de parâmetros a ser estimado .... \\
\hline$\sigma_{e}^{2}$ & variância do erro \\
\hline$\chi$ & dados observados para definição dos agrupamentos (clustering) \\
\hline$\psi_{\Delta}$ & nível de transferência de informação \\
\hline
\end{tabular}




\section{Lista de Siglas}

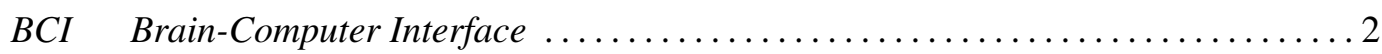

PSO Particle Swarm Optimization ................................ 2

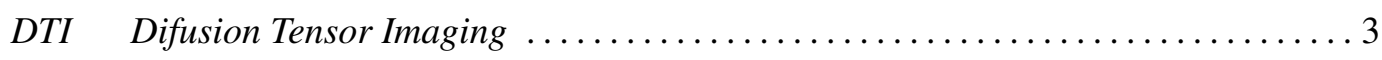

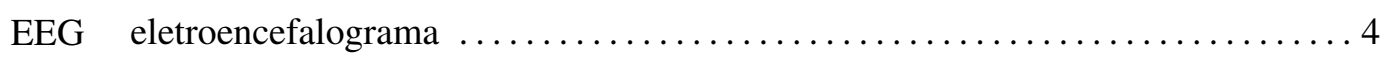

STA Spike Triggered Average ..................................... 5

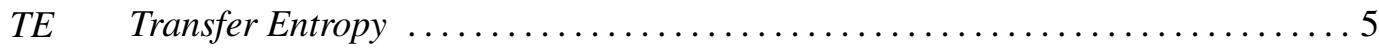

MI Mutual Information ...................................... 6

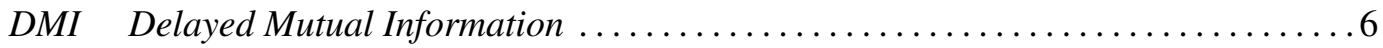

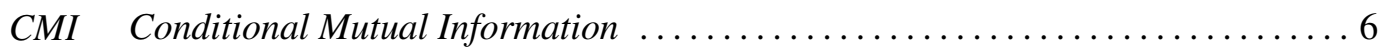

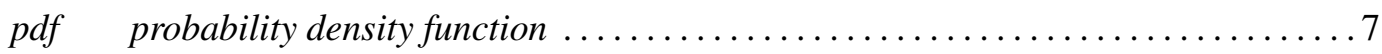

IAAFT Iterative Amplitude Adjusted Fourier Transform .................... 9

UK United Kingdom ........................................ 9

GWN Gaussian White Noise ..................................... 9

EPSP Excitatory Post Synaptic Potential ............................... 14

IPSP Inhibitory Post Synaptic Potential . ............................ 14

FeCO Femoral Chordotonal Organ .................................. 18

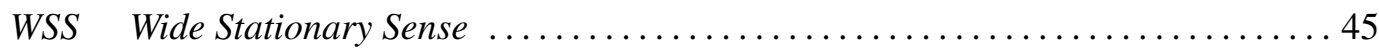

BIC Bayesian Information Criteria ................................69 


\section{Resumo}

\section{Endo, Wagner. Modelagem de Circuitos Neurais do Sistema Neuromotor e Proprioceptor}

de Insetos com o uso da Transferência de Informação entre conexões neurais. 2014. 153p. Tese. Escola de Engenharia de São Carlos, Universidade de São Paulo, São Carlos, 2014.

Apresenta-se, neste trabalho, o desenvolvimento de um modelo bioinspirado a partir do circuito neural de insetos. Este modelo é obtido através da análise de primeira ordem dada pelo STA (Spike Triggered Average) e pela transferência de informação entre os sinais neurais. São aplicadas técnicas baseadas na identificação dos atrasos de tempo da máxima coerência da informação. Utilizam-se, para esta finalidade, os conceitos da teoria de informação: a DMI (Delayed Mutual Information) e a TE (Transfer Entropy). Essas duas abordagens têm aplicação em transferência de informação, cada uma com suas particularidades. A DMI é uma ferramenta mais simples do que a $T E$, do ponto de vista computacional, pois depende da análise estatística de funções densidades de probabilidades de segunda ordem, enquanto que a $T E$, de funções de terceira ordem. Dependendo dos recursos computacionais disponíveis, este é um fator que deve ser levado em consideração. Os resultados de atraso da informação são muito bem identificados pela $D M I$. No entanto, a $D M I$ falha em distinguir a direção do fluxo de informação, quando se tem sistemas com transferência de informação indireta e com sobreposição da informação. Nesses casos, a TE é a ferramenta mais indicada para a determinação da direção do fluxo de informação, devido à dependência condicional imposta pelo histórico comum entre os sinais analisados. Em circuitos neurais, estas questões ocorrem em diversos casos. No gânglio metatorácico de insetos, os interneurônios locais possuem diferentes padrões de caminhos com sobreposição da informação, pois recebem sinais de diferentes neurônios sensores para o movimento das membros locomotores desses animais.

O principal objetivo deste trabalho é propor um modelo do circuito neural do inseto, para 
mapear como os sinais neurais se comportam, quando sujeitos a um conjunto de movimentos aleatórios impostos no membro do inseto. As respostas neurais são reflexos provocados pelo estímulo táctil, que gera o movimento na junção femorotibial do membro posterior. Nestes circuitos neurais, os sinais neurais são processados por interneurônios locais dos tipos spiking e nonspiking que operam em paralelo para processar a informação vinda dos neurônios sensores. Esses interneurônios recebem sinais de entrada de mecanorreceptores do membro posterior e da junção motora dos insetos. A principal característica dos interneurônios locais é a sua capacidade de se comunicar com outros neurônios, tendo ou não a presença de impulsos nervosos (spiking e nonspiking). Assim, forma-se um circuito neural com sinais de entradas (neurônios sensores) e saídas (neurônios motores).

Neste trabalho, os algoritmos propostos analisam desde a geração aleatória dos movimentos mecânicos e os estímulos nos neurônios sensores que chegam até o gânglio metatorácico, incluindo suas respostas nos neurônios motores. São implementados os algoritmos e seus respectivos pseudocódigos para a DMI e para a TE. É utilizada a técnica de Surrogate Data para inferir as medidas de significância estatística em relação à máxima coerência de informação entre os sinais neurais.

Os resultados a partir dos Surrogate Data são utilizados para a compensação dos erros de desvio das medidas de transferência de informação. Um algoritmo, baseado na IAAFT (Iterative Amplitude Adjusted Fourier Transform), gera os dados substitutos, com mesmo espectro de potência e diferentes distribuições dos sinais originais. Os resultados da $D M I$ e da $T E$ com os Surrogate Data fornecem os valores das linhas de base quando ocorre a mínima transferência de informação. Além disso, foram utilizados dados simulados, para uma discussão sobre os efeitos dos tamanhos das amostras e as forças de associação da informação.

Os sinais neurais coletados estão disponíveis em um banco de dados com diversos experimentos no gânglio metatorácico dos gafanhotos. No entanto, cada experimento possui poucos sinais coletados simultaneamente; assim, para diferentes experimentos, os sinais ficam sujeitos às variações de tamanho de amostras, além de ruídos que interferem nas medidas absolutas de transferência de informação. Para se mapear essas conexões neurais, foi utilizada a metodologia baseada na normalização e compensação dos erros de desvio para os cálculos da transferência de informação. As normalizações das medidas utilizam as entropias totais do sistema. Para a DMI, utiliza-se a média geométrica das entropias de $X$ e $Y$, para a $T E$ aplica-se a CMI(Conditional Mutual Information) para a sua normalização. 
Após a aplicação dessas abordagens, baseadas no STA e na transferência de informação, apresenta-se o modelo estrutural do circuito neural do sistema neuromotor de gafanhotos. São apresentados os resultados com o STA e a DMI para os neurônios sensores, dos quais são levantadas algumas hipóteses sobre o funcionamento desta parte do FeCO (Femoral Chordotonal Organ). Para cada tipo de neurônio foram identificados múltiplos caminhos no circuito neural, através dos tempos de atraso e dos valores de máxima coerência da informação. Nos interneurônios spiking obtiveram-se dois padrões de caminhos, enquanto que para os interneurônios nonspiking identificaram-se três padrões distintos. Esses resultados são obtidos computacionalmente e condizem com que é esperado a partir dos modelos biológicos descritos em Burrows (1996).

Palavras-chaves: neurociência motora, engenharia bioinspirada, STA, transferência de informação, transferência de entropia, nível de significância estatística, conectividade neural. 


\section{Abstract}

Endo, Wagner. Neural Circuits Modeling of Insects Neuromotor System based on Information Transfer Approach and Neural Connectivity. 2014. 153p. Thesis Doctoral. University of São Paulo at São Carlos School of Engineering, São Carlos, 2014.

Herein, we present the development of a bioinspired model by the neural circuit of insects. This model is obtained by analyzing the first order from STA (Spike Triggered Average) and the transfer of information among neural signals. Techniques are applied based on the identification of the time delays of the information maximum coherence. For this purpose we use the concepts of the theory of information: DMI (Delayed Mutual Information) and TE (Transfer Entropy). These two approaches have applications on information transfer and each one has peculiarities. The DMI is a simpler tool than the TE, from the computational point of view. Therefore, DMI depends on the statistical analysis of second order probability density functions, whereas the TE depends on third order functions. If computational resources are a problem, those questions can be taken into consideration. The results of the information delay are very effective for DMI. However, DMI fails to distinguish the direction of the information flow when we have systems subjected to indirect information transfer and superposition of the information. In these cases, the TE is the most appropriate tool for determining the direction of the information flow, due to the conditional dependence imposed by a common history among the signals. In neural circuits, those issues occur in many cases. For example, in metathoracic ganglion of insects, the local interneurons have different pathways with superposition of the information. Therefore, the interneurons receive signals from different sensory neurons for moving the animal's legs.

The main objective of this work is propose a model of the neural circuit from an insect. Additionally, we map the neural signals when the hind leg is subjected to a set of movements. 
Neural responses are reflexes caused by tactile stimulus, which generates the movement of femoro-tibial joint of the hind leg. In these neural circuits, the signals are processed by neural spiking and nonspiking local interneurons. These types of neurons operate in parallel processing of the information from the sensory neurons. Interneurons receive input signals from mechanoreceptors by the leg and the insect knees. The main feature of local interneurons is their ability to communicate with others neurons. It can occur with or without of the presence of impulses (spiking and nonspiking). Thus, they form a neural circuit with input signals (sensory neurons) and outputs (motor neurons).

The proposed algorithms analyze the random generation of movements and mechanical stimuli in sensory neurons. Which are processing in the metathoracic ganglion, including their responses in the motor neurons. The algorithms and the pseudo-code are implemented for TE and DMI. The technique of Surrogate Data is applied to infer the measures of statistical significance related to the information maximum coherence among neural signals.

The results of the Surrogate Data are used for bias error compensation from information transfer. An algorithm, based on IAAFT (Iterative Amplitude Adjusted Fourier Transform), generates Surrogate Data with the same power spectrum and different distributions of the original signals. The results of the surrogate data, for DMI and TE, achieve the values of baselines when there are minimum information transfer. Additionally, we used simulated data to discuss the effects of sample sizes and different strengths of information connectivity.

The collected neural signals are available from one database based on several experiments of the locusts metathoracic ganglion. However, each experiment has few simultaneously collected signals and the signals are subjected of variations in sample size and absolute measurements noisy of information transfer. We used a methodology based on normalization and compensation of the bias errors for computing the information transfer. The normalization of the measures uses the total entropy of the system. For the DMI, we applied the geometric mean of $X$ and $Y$. Whereas, for the TE is computed the CMI (Conditional Mutual Information) for the normalization.

We present the neural circuit structural model of the locusts neuromotor system, from those approaches based on STA and the information transfer. Some results are presented from STA and DMI for sensory neurones. Then, we achieve some new hypothesis about the neurophisiology function of FeCO. For each type of neuron, we identify multiple pathways in neural circuit through the time delay and the information maximum coherence. The spiking interneurons are 
yielded by two pathways, whereas the nonspiking interneurons has revealed three distinct patterns. These results are obtained computationally and are consistent with biological models described in Burrows (1996).

Keywords: motor neuroscience, bioinspired engineering, STA, transfer entropy, information flow, statistical significance level, neuronal connectivity, neural layers. 


\section{Capítulo 1}

\section{Introdução}

Atualmente, os sistemas bioinspirados têm ocupado um lugar de destaque em várias pesquisas, tanto de áreas da biologia quanto de áreas computacionais (Sporns, 2011). Este novo ramo de conhecimento reúne a biologia, a engenharia, a computação e a física (Zheng et al., 2013). E tem como principal característica a multidisciplinariedade. Essas pesquisas integram diferentes conhecimentos, para propor novas abordagens a problemas que necessitam outros direcionamentos na geração de novos resultados. Por exemplo, na utilização de redes dinâmicas complexas para a modelagem de atividades neurais (Bettencourt et al., 2008), no controle dinâmico não linear para o controle motor de próteses e exoesqueletos (Ying et al., 2007), nas ferramentas da inteligência computacional aplicadas a diferentes tipos de sistemas biológicos (Bjornsdotter e Wessberg, 2010) e nas abordagens bioinspiradas em detecção e extração de vírus de redes de computadores (Meisel et al., 2010).

A partir desse cenário, emerge a neurociência computacional com um papel muito relevante na fundamentação desses tipos de pesquisas. A neurociência computacional possui diferentes abordagens de sistemas neurais a partir do processamento de informações obtidas das estruturas que os compõem (DiCaprio, 2004). O processamento desses tipos de sinais utilizam ferramentas computacionais que funcionam como peças centrais para modelagem de sistemas bioinspirados. Essas técnicas possibilitam identificar diferentes tipos de circuitos neurais (Dewhirst et al., 2013), (Bettencourt et al., 2008) (Newland e Kondoh, 1997b). As suas principais aplicações têm impulsionado a curiosidade e atenção das pessoas em geral, pois possuem diversas abordagens no cotidiano, as quais vão desde a investigação do comportamento do cérebro, para um diagnóstico médico, até suas possibilidades de interação com máquinas e computadores, como a interface cérebro-máquina (BCI - Brain-Computer Interface) (Ang et al., 2012). São 
destacadas as aplicações de diferentes ferramentas em neurociência computacional, por exemplo, em McDonnell et al. (2011), o qual utiliza a teoria de informação e em Dewhirst et al. (2013) com abordagens não lineares.

Os estudos com insetos são promissores, pois servem de referência para os modelos aplicados na engenharia bioinspirada. São tomadas emprestadas, desde as estratégias de controle aplicadas a pequenos robôs, até o próprio comportamento descrito por enxames de insetos. Este comportamento é aplicado em algoritmos de inteligência computacional, como a otimização por enxame de partículas PSO - Particle Swarm Optimization (Bjornsdotter e Wessberg, 2010). Outro modelo emprestado vem da biomecânica motora de insetos que serve de referência para a engenharia de reabilitação e para o desenvolvimento de exoesqueletos e de próteses bioinspiradas (Jiménez-Fabián e Verlinden, 2012). Todas essas aplicações contribuem diretamente para a construção de modelos de circuitos neurais em suas abordagens.

Os circuitos neurais são formas de representação das associações entre diferentes partes de um sistema nervoso (Sporns, 2011). Na sua maioria, os circuitos neurais são obtidos através da análise de sinais (sinais bioelétricos) coletados a partir de um sistema neural. Esses sistemas podem ser estruturas complexas, como os cérebros de mamíferos ou estruturas locais com respostas autônomas, como é o caso dos reflexos de movimentos das pernas de insetos (Burrows, 1996). Diversos estudos utilizam redes complexas para o mapeamento da conectividade de diferentes partes de um sistema neural (Wilson, 1996), (Bettencourt et al., 2008) e (van Steen, 2010). Um exemplo em evidência, nos dias atuais, são os conectomas (Byrne, 1997) - na Figura 1.1 é apresentado o conectoma do cérebro humano. Este tema é exposto em detalhes em Sporns (2011).

Como a maioria das aplicações com circuitos neurais tem, em comum entre si, o processamento e análise de sinais, o aumento de sua complexidade faz surgir, constantemente, novas técnicas na literatura. São destacadas várias aplicações recentes, por exemplo, a determinação da significância da seletividade neural (Ince et al., 2012), a medida quantitativa de conectividade efetiva entre regiões do cérebro (Liu e Aviyente, 2012) e a determinação das associações da informação em processos multivariáveis (Faes et al., 2012). Essas técnicas são baseadas nas medidas de associação ou conectividade entre diferentes sinais, dados ou informações de um sistema neural. Além disso, esses estudos são aplicados em outras áreas do conhecimento, como em Nichols et al. (2005) para análise de sistemas estruturais mecânicos, e em Darbellay e Wuertz (2000) para séries temporais financeiras de bolsas de valores. Do ponto de vista de 


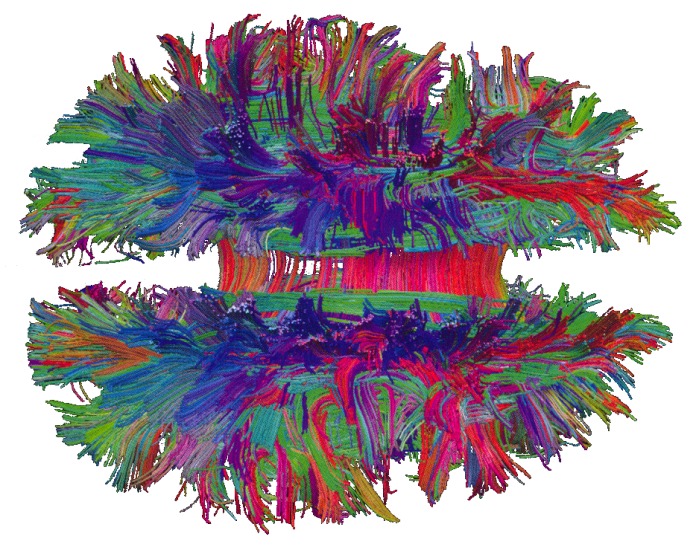

(a)

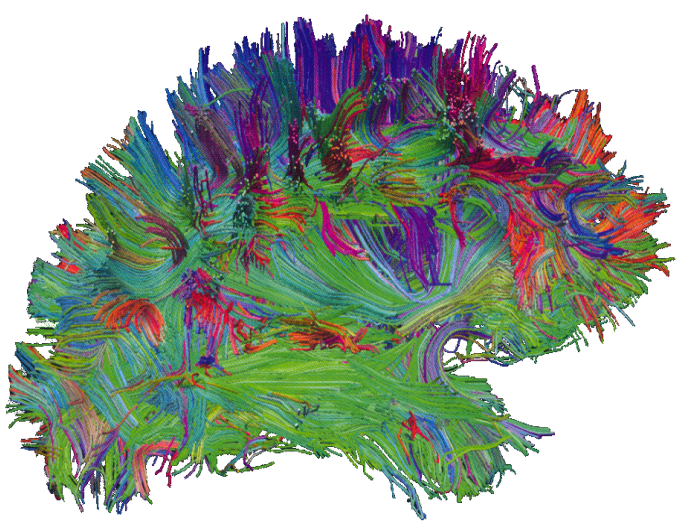

(b)

Figura 1.1: Exemplo da imagem de um conectoma do cerébro humano. Esta é uma aplicação de estudos de conectividade. Imagem por difusão espectral (DTI - Difusion Tensor Imaging) da vista (a) dorsal e (b) lateral do cérebro. [Fonte: Sporns (2011), p. 92 (plate 4)]

processamento de sinais e modelagens de sistemas, estas ferramentas se tornam promissoras à medida que são adaptadas a cada uma das especificidades impostas para cada aplicação.

Neste trabalho, a metodologia para a aplicação de sistemas bioinspirados está voltada para o desenvolvimento de modelos de circuitos neurais de insetos, especificamente o sistema proprioceptor. A propriocepção ou cinestesia é definida como qualquer informação postural ou posicional, encaminhada ao sistema nervoso central por receptores encontrados em músculos, tendões, ligamentos ou articulações. Sendo sua principal função, transmitir a consciência dos movimentos produzidos pelos membros posteriores ou inferiores através de neurônios sensores (Romero, 2000). O sistema proprioceptor está presente tanto em insetos quanto em mamíferos e possui modelos análogos (Fouad et al., 2003). Tal como a estrutura neural presente em insetos denominada, $\mathrm{FeCO}$ (Femoral Chordotonal Organ), que é responsável por identificar movimentos de flexão e extensão na junção femorotibial do membro posterior do inseto (Burrows et al., 1988). Esta estrutura possui comportamento funcional equivalente ao fuso muscular (muscle spindles) dos mamíferos (Fouad et al., 2003). Os modelos estudados em Vidal-Gadea et al. (2010) citam que o comportamento neuromotor de insetos é muito similar a sistemas neurais mais complexos. Os tipos de sinais neurais e a forma como são processados nos neurônios compartilham diversas características. Um processo bastante similar é o de inibição e excitação dos músculos através da propriocepção. Este processo envolve neurônios aferentes, que são aqueles que enviam informação periférica ao sistema nervoso central (Romero, 2000). O padrão de caminho percorrido pelos sinais envolve neurônios sensores, motores e internos (interneurônios). Uma importante característica para a análise de circuitos neurais, ao se utilizar 
insetos, é a viabilidade experimental de se obter sinais intracelulares, conforme são descritos nos procedimentos de Newland e Kondoh (1997a) e Newland e Kondoh (1997b). Pois, em muitos casos, a partir desse tipo de análise consegue-se coletar as ações de potencial geradas diretamente nos neurônios.

As estruturas neurais de insetos são simples e possuem numericamente poucos neurônios, quando comparados ao sistema neural de mamíferos. No gânglio metatorácico de um gafanhoto existem cerca de 900 a 1500 neurônios que controlam todos os movimentos da membro posterior. Sabe-se que esse pequeno grupo de neurônios é responsável por todos os movimentos de reflexos locais, como caminhar e saltar (Vidal-Gadea et al., 2010). A possibilidade de se estudar circuitos neurais simples e com funcionamento similar a estruturas mais complexas atrai muita atenção para pesquisas em neurociência motora de insetos.

Diversos estudos buscam analisar circuitos neurais que estabelecem relações de associação entre os sinais obtidos. Por exemplo, algumas aplicações envolvem medidas de associação entre a percepção audiovisual e a ativação de partes do sistema neural, após a ocorrência desses estímulos (Ito et al., 2011). Outras abordagens são aplicadas em sinais de eletroencefalograma (EEG) que buscam áreas de associação entre regiões distintas do cérebro (Greenblatt et al., 2012). Os modelos de circuitos neurais são descritos a partir de medidas de associações anatômicas, funcionais ou efetivas (Lee et al., 2012). Uma outra abordagem que tem recebido bastante atenção é a identificação de tempos de atraso da informação em sistemas biológicos (Silchenko et al., 2010). Os tempos de atraso são obtidos de diferentes formas, uma delas é pelo índice de decaimento de fase (Nolte et al., 2008 apud Yang et al. (2013)), e outra pelo atraso da máxima coerência da informação (Wilmer et al., 2012). A partir desses tempos de atraso e de um conhecimento fisiológico sobre o circuito neural, é possível descrever os múltiplos caminhos de um sinal. Em Burrows (1987) e Burrows et al. (1988) são descritos múltiplos caminhos de processamento paralelo dos sinais, ocasionados pelo estímulo do movimento em um membro do inseto.

As principais abordagens deste trabalho buscam determinar os modelos de circuitos neurais utilizando as ferramentas da teoria de informação (Cover e Thomas, 2006), incluindo as análises estatísticas dos sinais e da obtenção de medidas de associação (Schwartz et al., 2006). Um circuito neural pode ser abordado como um sistema de codificação e decodificação de sinais. Pela teoria de informação, as medidas são feitas através da estimativa de suas incertezas. Essas medidas estão relacionadas à definição desses conceitos em 1948, em 
Shannon (1948). A fundamentação original vem da entropia de Boltzmam, da Física, sendo que Shannon reinterpretou-a para sistemas de comunicação. De acordo com Cover e Thomas (2006), a entropia de um sinal é descrita através de variáveis aleatórias discretas em: $H(X)=-\sum_{x \in \phi} p(x) \log _{2} p(x)$, onde $\phi$ representa o conjunto de símbolos discretizados usados na codificação do conjunto da variável aleatória $X, p(x)$ é a probabilidade de eventos $x \mathrm{e}$ $H(X)$ quantifica o número médio de bits (quando se utiliza a base 2 no $l o g$ ) que representa os estados de incerteza da variável aletatória, $X$.

Para a aplicação em circuitos neurais, um dos principais objetivos é medir a associação ou informação compartilhada entre dois ou mais biossinais (Dayan e Abbott, 2001). Os parâmetros estatísticos, vindos das funções densidade de probabilidades ( $p d f)$, são importantes na análise de transferência de informação, pois é a partir delas que as estimativas das medidas de associação são quantificadas (Lee et al., 2012). As medidas estatísticas de primeira ordem utilizam, principalmente, valores médios associados a um evento. Em circuitos neurais, uma importante ferramenta é a análise das curvas de Spike-Triggered Average (Schwartz et al., 2006), (Pillow e Simoncelli, 2006). Matematicamente, a STA é o estímulo médio anterior um pico (spike). Para se calcular o STA, a janela de tempo do estímulo anterior a cada spike é obtida ao se identificar todas as suas ocorrências em um sinal neural. No entanto, a análise de STA é indicada ao se analisar neurônios nos quais existem ocorrências de spikes (Schwartz et al., 2006). Ao se obter uma curva média, dada pelo STA, tem-se uma importante medida de associação. Do ponto de vista estatístico, trata-se de uma análise de primeira ordem (valores médios) (Park e Pillow, 2011).

Uma outra ferramenta, bastante recente, que descreve as direções de associação e a quantidade de informação compartilhada é a transferência de entropia (TE - Transfer Entropy), apresentada por Schreiber (2000). Essa abordagem mostrou-se promissora, pois foi aplicada em áreas da neurociência (Gourévitch e Eggermont, 2007), nas áreas de economia e finanças (Marschinski e Kantz, 2002) e até em estudos de dados climáticos (Runge et al., 2012).

A TE surgiu como uma generalização da informação mútua (MI - Mutual Information) (Schreiber, 2000). A MI é definida como uma medida de associação que quantifica a informação compartilhada entre variáveis aleatórias (Cover e Thomas, 2006). A informação mútua é semelhante à análise de correlação cruzada linear, com a diferença de ser aplicada diretamente para funções não monótonas ou não lineares (Ince et al., 2012). No caso de circuitos neurais, as medidas de associação da informação são composições de diferentes tipos de funções, 
incluindo as lineares e não lineares (Jin et al., 2010).

A informação mútua também é utilizada para se medir a direção de transferência de informação (Alonso et al., 2007). Para isso adiciona-se um tempo de atraso, $\tau$, denominado de atraso da informação mútua (DMI - Delayed Mutual Information). A determinação do atraso na informação mútua define uma direção de fluxo dessa informação. Para aplicações em medidas de associação, é feita uma estimativa da $M I$ no intervalo, $-t \leq \tau \leq t$. O tempo de atraso, $\tau$, em que ocorre o valor de máxima coerência da informação mútua, representa a medida de associação efetiva entre duas séries temporais de um circuito neural (Dionisio et al., 2004). Na estimativa da $D M I$, a definição do tempo de atraso indica a direção desse fluxo. Em outras palavras, se o valor do tempo de atraso for definido para $\tau>0$, o fluxo de informação ocorre em $X \rightarrow Y$. Em oposição, para um valor de $\tau<0$, ocorre o fluxo de informação em $Y \rightarrow X$.

No entanto, em alguns casos de bidirecionalidade da informação, a $D M I$ falha em distinguir a direção efetiva da transferência de informação (Schreiber, 2000). A DMI apenas mede a informação comum entre dois sinais, sem uma medida dinâmica de transferência de informação efetiva. A Transferência de Entropia, introduzida por Schreiber (2000), é interpretada em termos de uma informação mútua condicionada CMI-Conditional Mutual Information (Lindner et al., 2011) baseada no histórico comum dos sinais. Na Figura 1.2 é mostrado o exemplo da medida da associação e da direção da transferência de informação entre duas séries temporais $Y$ e $Z$ com o histórico comum em $X$. Nesses casos, as medidas de associação pela $M I$ padrão não distinguem a ocorrência de sobreposição das direções do fluxo de informação. Em experimentos práticos em circuitos neurais, isso ocorre quando se tem em comum um sinal de excitação (mecânica, motora ou bioelétrica) se ramificando em múltiplos caminhos.

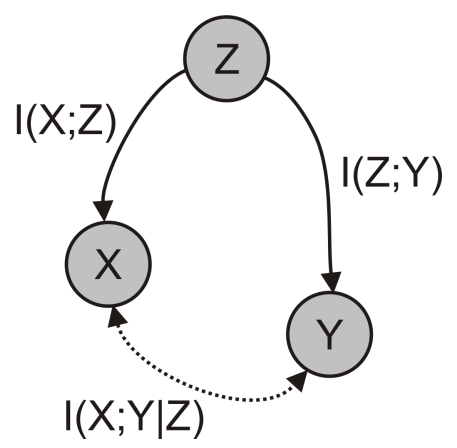

Figura 1.2: Modelo de conectividade com histórico comum de informação. Os efeitos de conectividade podem ser detectados entre partes de um sistema, com a propagação aos próximos estágios de um circuito neural. 
Expandindo o conceito da $C M I$ para a $T E$, estima-se o quanto de informação é reduzida ao se utilizar o histórico comum de uma das séries temporais, obtendo-se, assim, a medida da direção efetiva da transferência de informação. A Figura 1.3 mostra, através dos diagramas de Venn, os efeitos da $C M I$ sobre a $M I$ padrão. Pois, ao se inserir o histórico comum, reduz a incerteza da $D M I$, como visto na Figura 1.3(b), para a $T E$.

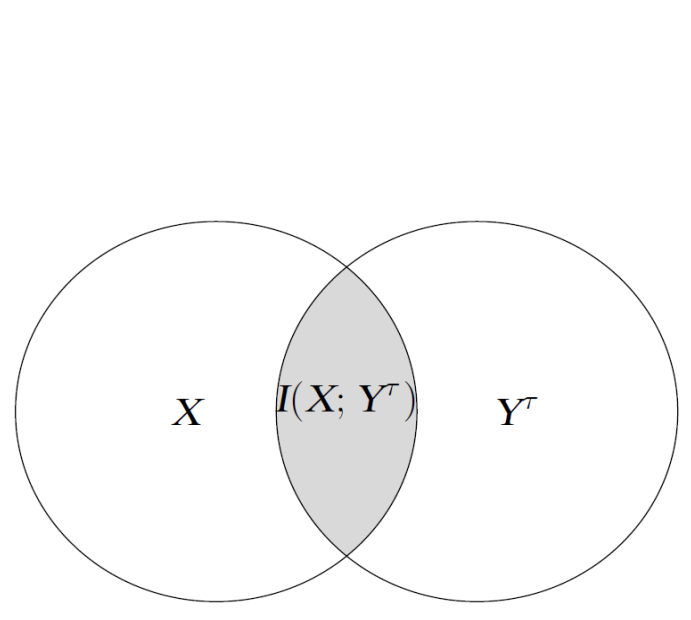

(a)

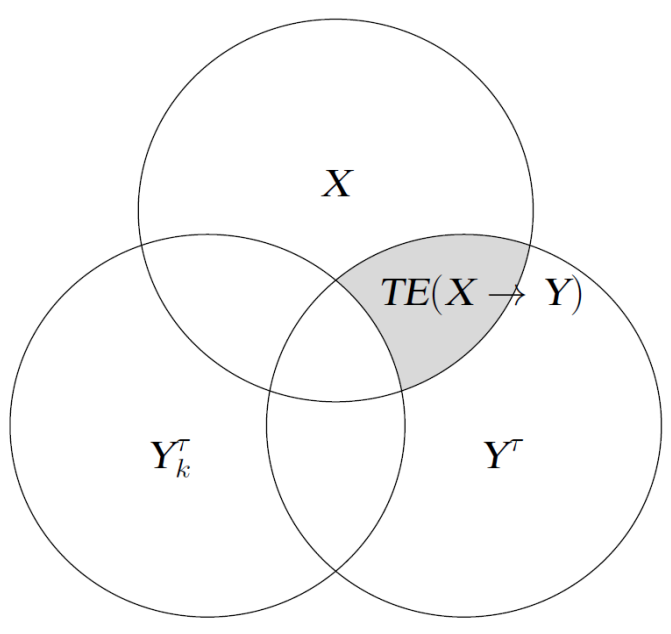

(b)

Figura 1.3: Diagrama de Venn da (a) informação mútua e da (b) transferência de entropia com um atraso, $\tau$. A área de intersecção (cinza claro) representa a redução da incerteza da informação compartilhada entre duas variáveis aleatórias, dado o conhecimento do passado na direção de transferência de entropia. No caso, de $X$ para $Y$, onde $k$ é um bloco anterior da variável aleatória $Y$.

A principal consequência da $T E$ é ser assimétrica, ao contrário do que ocorre na $D M I$. Assim, a estimativa da $T E$, de $X$ para $Y$, é diferente do caminho inverso, de $Y$ para $X$, sendo $k$ um bloco do histórico comum entre as séries temporais. A informação condicionada é definida pelo tempo anterior $Y_{k}$ ou $X_{k}$, dependendo da direção que se deseja medir a TE. Na Figura 1.4 é apresentado um exemplo da análise de associação entre duas variáveis com um tempo atraso, $\tau$, aplicado para a $T E(X \rightarrow Y)$. A direção de transferência de informação é importante, quando os circuitos neurais possuem múltiplos caminhos ou informação com fluxo bidirecional (Yang et al., 2013).

Por outro lado, a transferência de entropia pode ter alguma desvantagem em relação à informação mútua atrasada. Nos cálculos para obtenção da informação mútua e da transferência de entropia, essas medidas são baseadas nas funções densidade de probabilidades ( $p d f$ - probability density function) dos sinais. A complexidade das $p d f$ 's cresce de forma exponencial (Runge et al., 2012), pois, para um sistema com duas variáveis, é necessário uma $p d f$ de se- 


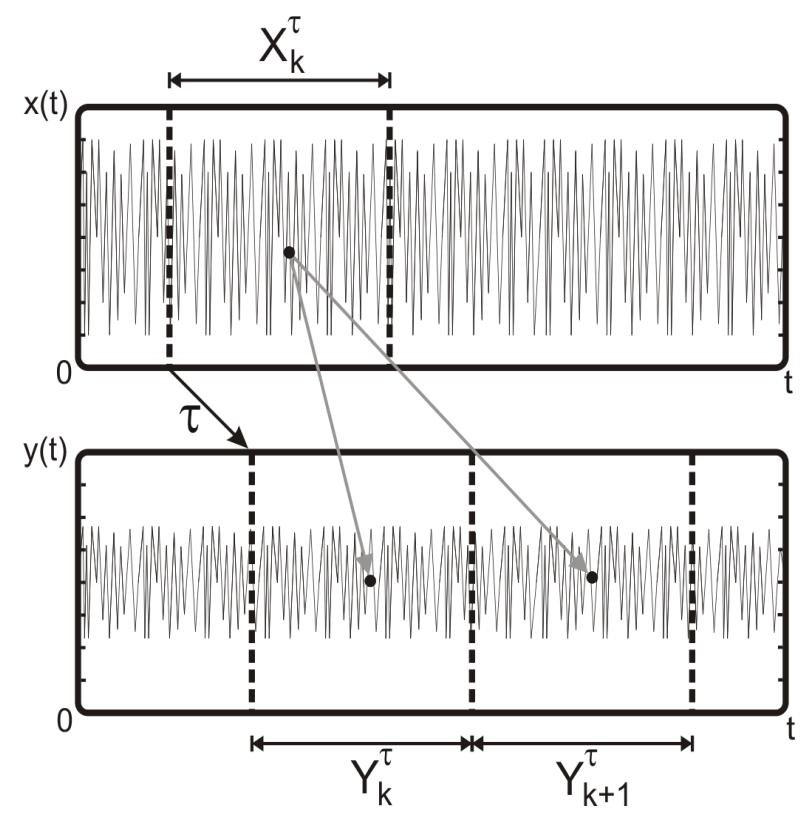

Figura 1.4: Exemplo de análise da transferência de entropia (TE). São representadas as definições dos trechos de análise para a TE.

gunda ordem representada em dois eixos. Quando o sistema utiliza uma $p d f$ de terceira ordem, é necessária uma matriz de 3 dimensões e assim por diante. Do ponto de vista computacional, isto é uma desvantagem para a sua representação e para a obtenção de dados, pois a alocação de memória e o tempo de processamento exigirão da parte computacional. Para se ter uma ideia, considera-se uma variável aleatória com distribuição uniforme utilizando 4 bits de resolução. Para a TE, seriam necessários no mínimo 64 bits para representar a $p d f$ de três dimensões dessa variável aleatória. Enquanto que, para a $D M I$, seriam necessários pelo menos 16 bits. Caso esses requisitos não sejam atendidos, o cálculo dessas funções perde informação e, consequentemente, ocorrem erros no resultado final.

Todas as abordagens para o estudo dos circuitos neurais foram comparadas, utilizando-se a técnica de Surrogate Data (Schreiber e Schmitz, 1996). A ideia central é substituir uma série temporal por outra similar, com as mesmas amplitudes e mesmo espectro de potência (Schreiber e Schmitz, 2000). O resultado dessa técnica significa que, ao se descorrelacionar as fases das amplitudes dos sinais analisados, eles perdem suas medidas de associação (Venema et al., 2006). Ao se comparar as novas medidas com o Surrogate Data e as séries temporais originais, é possível analisar-se a significância estatística da quantidade de associação da informação. Esse teste é aplicado entre os picos de máxima coerência e as linhas de base geradas pelo Surrogate Data; assim, evita-se a identificação de falsos positivos. Existem, na literatura, alguns algoritmos que abordam metodologias para a geração de Surrogate Data, como em Kugiumtzis 
(2002) e Dolan e Spano (2001). Utilizaram-se, para a geração do Surrogate Data, os conceitos baseados no ajuste iterativo de amplitude pela transformada de Fourier (IAAFT - Iterative Amplitude Adjusted Fourier Transform) de Venema et al. (2006).

Neste trabalho são apresentadas as técnicas de estimativa das $p d f$ 's e os pseudocódigos dos algoritmos para obtenção das curvas de $S T A$, do cálculo da $D M I$ e da $T E$, incluindo ainda uma rotina para a geração dos Surrogate Data. Foram gerados dados simulados para testes e demonstração de resultados. Nesses tipos de dados, foi possível analisar o efeito de amostras de sinais com tamanhos variados e sua influência na significância dos resultados finais. Ao se selecionar, arbitrariamente, trechos estacionários das séries temporais, os resultados de máxima coerência de informação sofrem os efeitos dessas variações. Para este problema na seleção dos sinais, foram propostas medidas de normalização (Ince et al., 2012) e de compensação desses erros de desvio (Gourévitch e Eggermont, 2007).

Os dados reais foram coletados de experimentos obtidos de gafanhotos do deserto, conforme referências descritas em Kondoh et al. (1995), Newland e Kondoh (1997a), Newland e Kondoh (1997b) e Vidal-Gadea et al. (2010). Esses experimentos foram realizados na Universidade de Southampton, UK, pelo grupo de pesquisa do professor Philip L. Newland. Todos os sinais são coletados entre os interneurônios do gânglio metatorácico e os neurônios motores do membro traseiro do gafanhoto. Uma excitação mecânica aleatória, com largura de banda de $27 \mathrm{~Hz}$, é utilizada para a movimentação do apódema da junção femorotibial do inseto. Este é um sinal gaussiano(GWN - Gaussian White Noise) que simula os movimentos da junção, entre o fêmur e a tíbia, da membro posterior do gafanhoto. Os procedimentos experimentais para a coleta dos dados são melhor detalhados no Capítulo 4.

O trabalho busca aplicar os conceitos de transferência de informação, através dos tempos de atrasos e da máxima coerência de associção, os quais são obtidos com os algoritmos da $D M I$ e da TE. Além de apresentar outras medidas estatísticas de associação, através do STA e da análise das $p d f$ condicionais, com auxílio de regras bayesianas. Desta forma, são obtidos o mapeamento e o modelo de associação e de transferência de informação do circuito neural do sistema propriceptor, a partir do gânglio metatorácico de gafanhotos. 


\subsection{Objetivos}

O objetivo deste trabalho é propor um modelo da associação e transferência de informação baseado nos sinais neurais do gânglio metatorácico, dos neurônios sensores e motores de insetos. Para isso, são desenvolvidos algoritmos para o atraso da informação mútua, transferência de entropia e a geração de Surrogate Data.

\subsubsection{Objetivos específicos}

A partir do foco central deste trabalho, são definidos objetivos específicos para a obtenção do mapeamento da transferência de informação e o modelo estrutural do circuito neural do sistema neuromotor:

1. Analisar de forma conjunta os dados e definir os múltiplos padrões de caminhos dos sinais de parte do sistema neuromotor de gafanhotos;

2. Modelar um sistema parcialmente conhecido com o uso de ferramentas da teoria de informação baseada nos circuitos neurais de insetos;

3. Compatibilizar as medidas de transferência de informação de diferentes experimentos.

4. Apresentar e discutir um modelo geral do gânglio metatorácico para o sistema neuromotor da membro posterior do gafanhoto.

\subsection{Estrutura do trabalho}

Para a revisão bibliográfica do trabalho, são apresentados os Capítulos 2 e 3. O Capítulo 2 apresenta um breve estudo sobre as características neurais e biológicas do sistema neural de insetos. Além de apresentar algumas referências da neurociência computacional aplicadas a estes modelos. No Capítulo 3 é feita uma revisão dos principais conceitos de medidas de associação. Inluindo os conceitos de $S T A$, da correlação cruzada e da teoria de informação, focando principalmente a transferência e máxima coerência de informação. Em seguida, no Capítulo 4, são apresentadas as metodologias e os algoritmos para as estimativas das $p d f$ 's, obtenção da curva média pelo $S T A$, da informação mútua atrasada e da transferência de entropia. O algoritmo para geração do Surrogate Data é desenvolvido para a comparação e validação de resultados. 
São descritos os modelos dos dados simulados e dos dados reais. Por fim, no Capítulo 5, são feitas as discussões e apresentação dos principais resultados para os dados simulados e para os dados reais. É apresentado e discutido o modelo e mapeamento estrutural do circuito neural do sistema neuromotor do gafanhoto. No Capítulo 7, as principais conclusões deste trabalho são apresentadas, bem como as contribuições e publicações obtidas. 


\section{Capítulo 2}

\section{Sistema neuromotor de um inseto}

Neste capítulo, apresenta-se um breve estudo de neurofisiologia comparada. São apresentadas as principais características neurofisiológicas, desde a unidade celular, até o modelo de circuitos neurais presentes nas respostas do sistema proprioceptor. Inclui-se a descrição dos modelos análogos, entre a neurofisiologia de insetos e a de sistemas neurais mais complexos. Nas subseções são tratadas, em particular, as características do sistema neuromotor de um gafanhoto. São descritos a estrutura do sistema neural, os tipos de neurônios e seus respetivos sinais característicos, como: spiking e nonspiking.

\subsection{Uma breve introdução à neurofisiologia}

Este breve estudo de neurofisiologia mostra o que existe em comum entre os diferentes tipos de sistemas nervosos, sejam de vertebrados ou invertebrados. Deve-se entender que o modelo de um neurônio é muito similar em diferentes animais. Desta forma, define-se um neurônio como uma célula neural que conecta e compõe diferentes partes de um sistema neural. Ele é responsável pela condução bioelétrica dos sinais entre os neurônios e é constituído basicamente de três partes: dentritos, axônio e sinapses.

Para melhor compreender as redes neurais, é necessário entender as formas como os neurônios se comunicam entre si. Isso ocorre através das conexões sinápticas, e o seu processo é chamado de transmissão sináptica. O sinal é recebido pelos dentritos e transmistido através do axônio; em seguida, ocorre a conexão pós-sináptica para um outro neurônio. A Figura $2.1^{1}$

\footnotetext{
${ }^{1}$ Original disponível em: http://nba.uth.tmc.edu/neuroscience/
} 
representa um exemplo de conexão pós-sináptica através da presença de neurotransmissores.

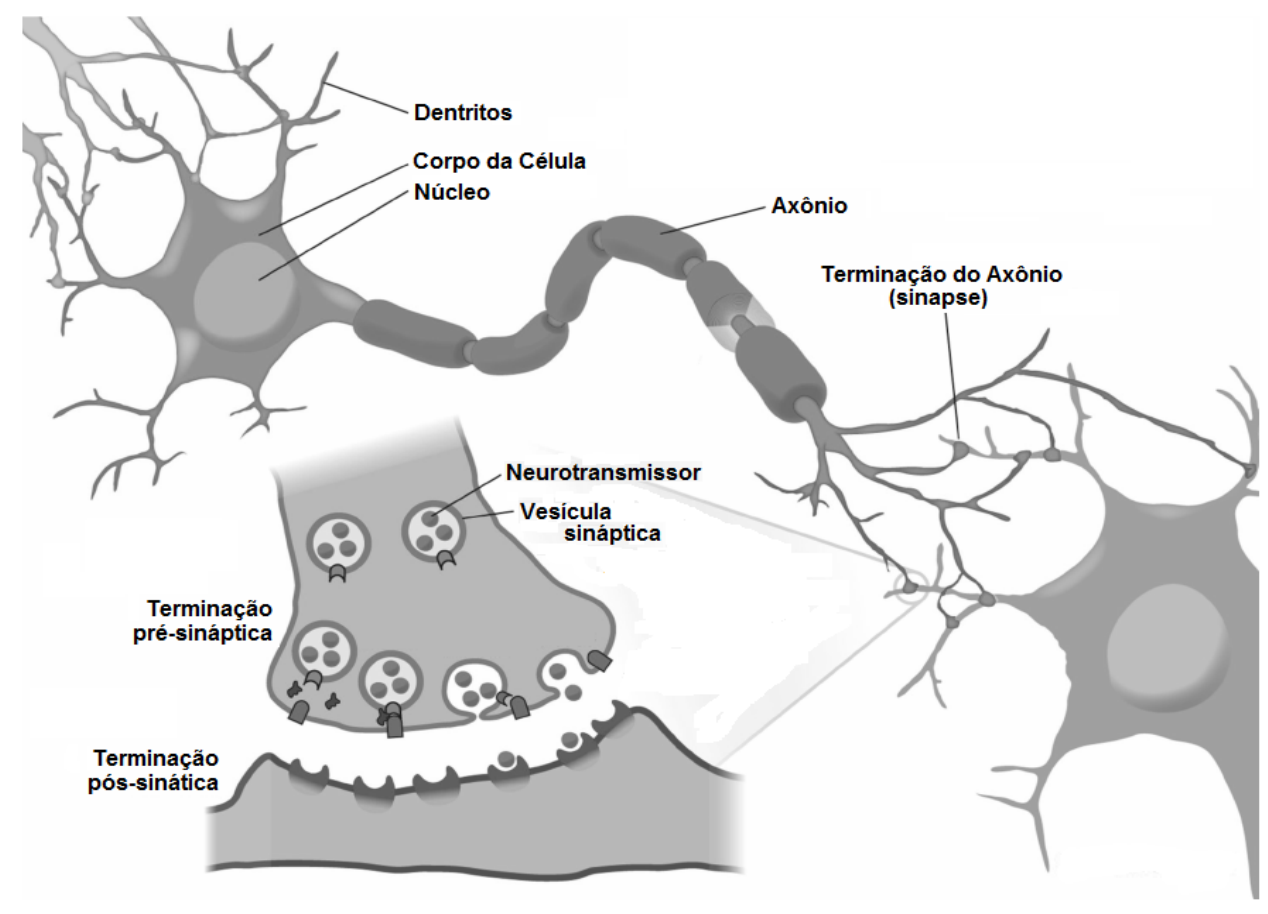

Figura 2.1: Representação de uma conexão sináptica, com as partes de um neurônio. [Adaptada de Byrne (1997)]

No interior dos neurônios ocorre, através do axônio e do corpo celular, a geração de potenciais elétricos denominados, potenciais de ação. A diferença de concentração gerada pelo corpo celular do neurônio produz um potencial elétrico, denoninado potencial de ação. Este, por sua vez, produz um efeito de depolarização no interior da célula, através da mudança de concetração de íons (cátions - $\mathrm{Na}^{+}$ou $\mathrm{K}^{+}$) entre o meio externo e interno do neurônio. Os potenciais da depolarização são cumulativos e, quando se tornam suficientemente grandes, disparam uma ação de potencial, resultando em um impulso bioelétrico, também conhecido como spike (Byrne, 1997).

Os potenciais de ação nas células neurais definem o tipo de sinal enviado, nos quais ocorrem as transmissões sinápticas. Os tipos de sinais dependem de como as células são excitadas ou inibidas, quando sujeitas a diferentes potenciais de ação. Uma forma de ocorrência da transmissão sináptica está relacionada com o potencial excitatório pós-sináptico (EPSP - Excitatory Post Synaptic Potential) ou com o potencial inibitório pós-sináptico (IPSP - Inhibitory Post Synaptic Potential). Os potenciais de ação devem ser coordenadas entre si, pois o processamento desse tipo de informação é fundamental na sincronia de movimentos em um músculo ou conjunto deles. Os potenciais têm que ser capazes de excitar e inibir, simultaneamente, músculos 
atuantes na movimentação de um membro ou alguma parte do corpo. A partir desse modelo sequencial de processamento de informações, forma-se o que se denomina um circuito neural (Sporns, 2011).

Tanto a EPSP quanto a IPSP ocorrem pela presença, ou não, da diferença de potencial elétrico gerado pelas concentrações de íons entre a parte externa e interna dos neurônios. Todas as etapas envolvidas entre os sinais sinápticos são representadas utilizando-se um modelo de uma rede de conexões (micronetworks motif) (Byrne, 1997) para a modelagem de circuitos neurais, com os quais são determinados os potenciais de excitação e inibição, através dos potenciais de ação. Na Figura 2.2, é apresentada uma topologia de um circuito neural com típicas redes de conexões sinápticas. Apresentam-se algumas topologias como uma rede de divergência e convergência, uma de excitação e outra de inibição por antecipação.

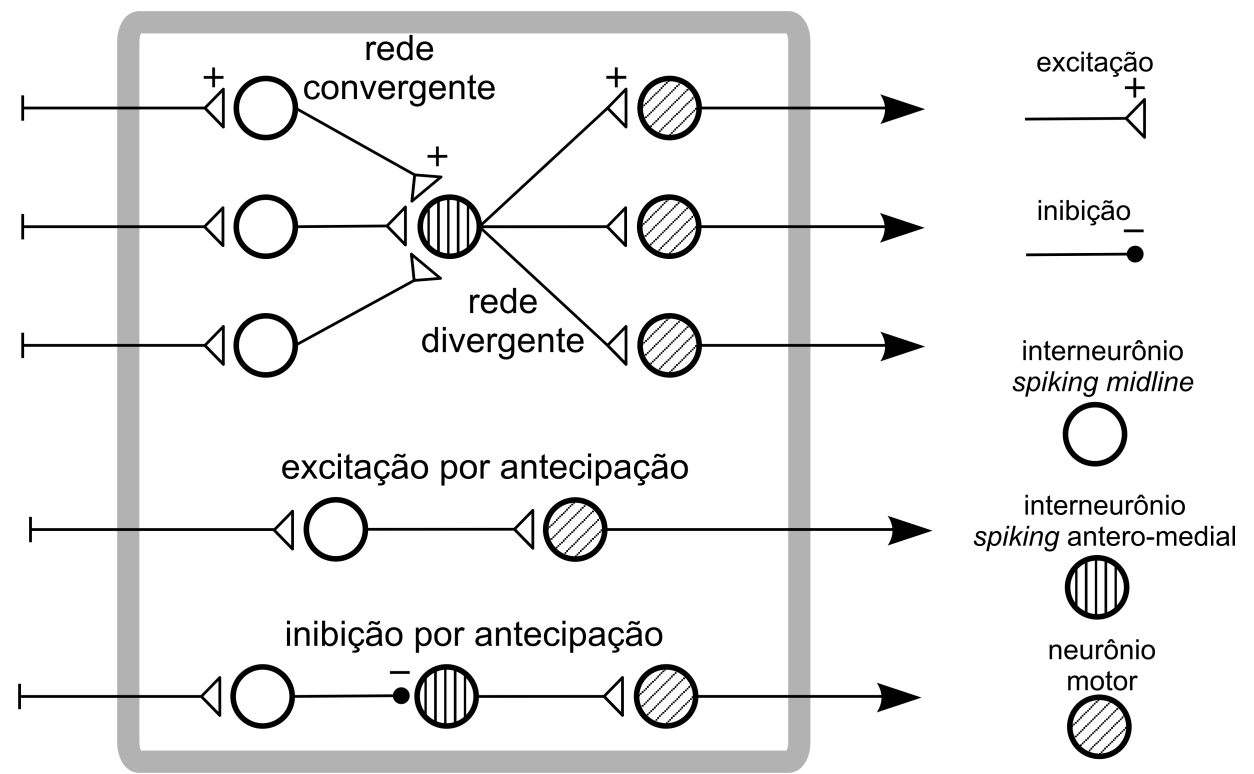

Figura 2.2: Exemplo de um circuito neural com típicas redes de conexões sinápticas. Apresentam-se algumas topologias: rede de divergência e convergência, excitação e inibição por antecipação.

As topologias das redes de circuitos neurais seguem uma sequência de potenciais que são compostas de inibição ou excitação. Esses potenciais possuem efeito de sobreposição; isso significa que uma sequência de duas inibições, por exemplo, gera uma excitação. Esse efeito ocorre em nível celular, na transmissão de um sinal bioelétrico entre neurônios. 


\subsection{Processamento e controle local dos membros locomotores}

Os modelos de circuitos neurais, abordados neste trabalho, concentram-se no estudo do sistema neuromotor e, especificamente, o proprioceptor. Este sistema é responsável pelo processamento e controle local dos membros de um inseto.

O processamento dos sinais percorre uma sequência de eventos na geração de força muscular em um sistema neuromotor, conforme é visualizado na Figura 2.3. Este sistema recebe sinais de diferentes circuitos neurais sensores. A partir dos quais são processados e controlados pelo sistema nervoso central ou pelos neurônios locais (Devasahayam, 2013).

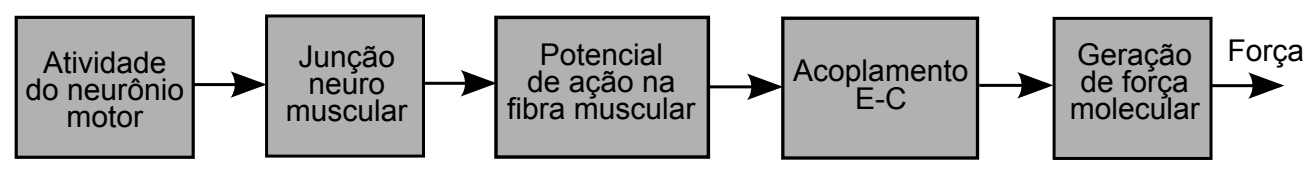

Figura 2.3: Diagrama de geração da força muscular em um sistema neuromotor. [Adaptada de Devasahayam (2013)]

Desta forma, definem-se partes distintas responsáveis pelo processamento dos sinais, pelo controle local e pela geração de força mecânica envolvidas no processo de movimento dos membros de um inseto. Uma importante forma de controle local de movimentos é realizada pelo sistema proprioceptor.

\subsubsection{Estudo comparado de controle e processamento do sistema proprioceptor}

A propriocepção é definida como sendo qualquer informação neural referente a posição, velocidade ou força de um membro enviadas ao sistema nervoso central. Esses elementos sensores são encontrados em diferentes partes do inseto ou mamífero, como em músculos, tendões, ligamentos, articulações e até mesmo a pele (Romero, 2000). Em outras palavras, é a consciência dos movimentos produzidos pelos nossos membros.

Ao se falar sobre propriocepção, é necessário descrever os receptores sensoriais, responsáveis por transmitir os sinais neurais ao sistema nervoso central. Os receptores sensoriais são encontrados no sistema nervoso somático, respondendo pelas distintas ações que são geradas a partir do movimento dos membros.

Um dos desafios da última década envolve o desenvolvimento de interfaces cérebro-computador bidirecionais (Bi-BCI). Na Figura 2.4 é mostrado o modelo bidirecional de uma Bi-BCI. Nesse modelo, busca-se desenvolver um sistema capaz de perceber os próprios movimentos da ação 
motora e o reconhecimento propriceptor que realimenta a sensação espacial para o cérebro, a partir do movimentos mecânicos da prótese.

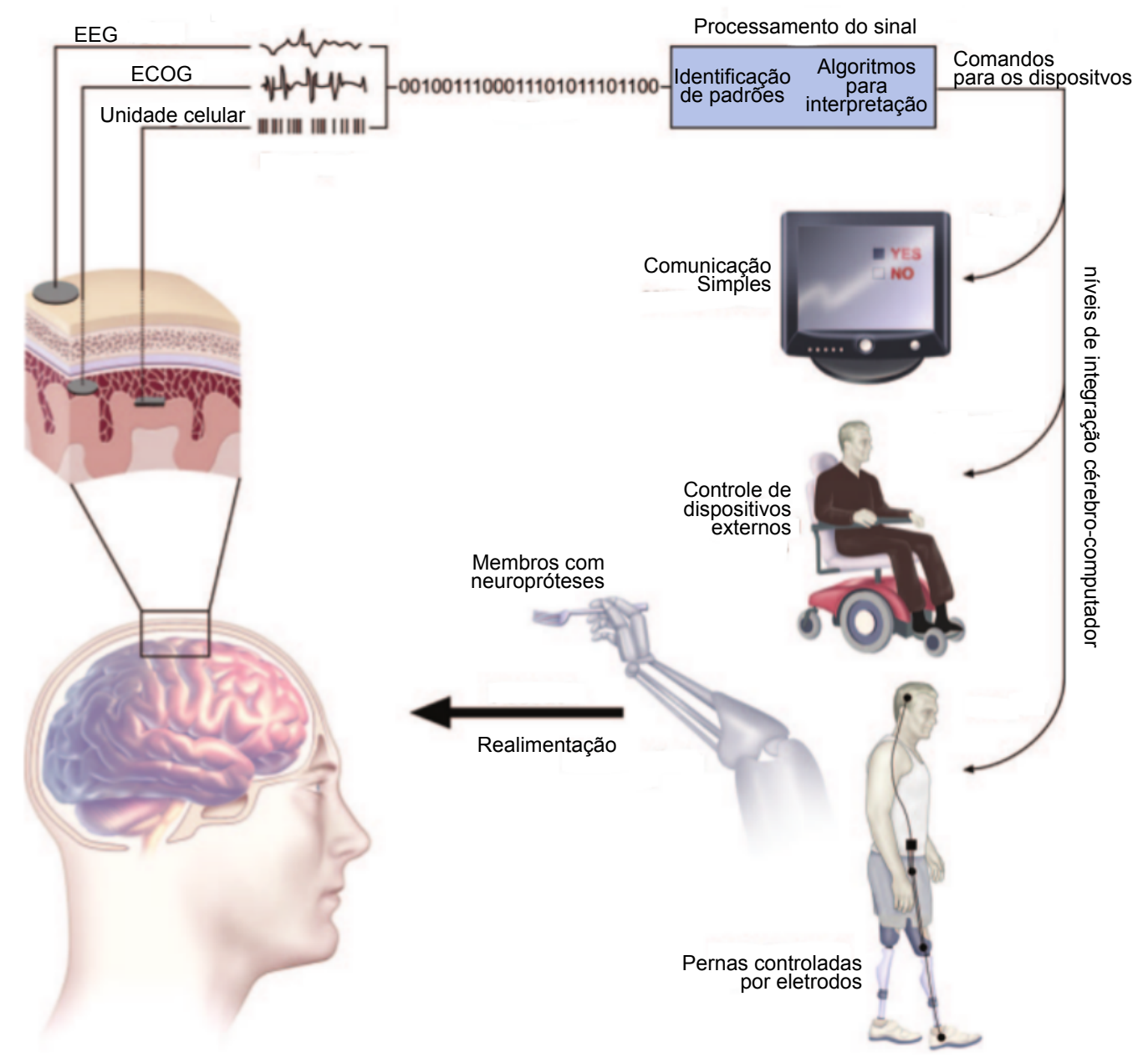

Figura 2.4: Representação de uma interface cérebro-computador BCI bidirecional, composta pelo elemento final de controle (a prótese mecânica) e os circuitos sensores de realiamentação em diversos níveis de interface: eletroencefalograma $(E E G)$, eletrocorticografia $(E C o G) \mathrm{e}$ unidades celular (single unit). [Adaptada de Leuthardt et al. (2006)]

O estudo dessas estruturas presentes no sistema proprioceptor de insetos e mamíferos tornase relevante ao compará-los e modelá-los, a fim de se conhecer como os sinais de propriocepção são gerados e sentidos no sistema nervoso central. Assim, podem-se destacar algumas estruturas sensoriais envolvidas na propricepção dos movimentos e que estão presentes em insetos ou mamíferos.

Uma importante estrutura é conhecida como sensila campaniforme (Campaniform Sensillae), a qual possui seu modelo análogo em vertebrados, o órgão tendinoso de Golgi (GTO Golgi tendon organ) (Fouad et al., 2003). A sensila campaniforme detecta a carga aplicada 
pelo músculo, a partir da deformação mecânica da cutícula (exoesqueleto), ao se executar o movimento de um membro. Enquanto que, nos vertebrados, o GTO detecta a deformação diretamente na extremidade do músculo, a qual está associada à intensidade de força aplicada sobre o membro (Burrows, 1996). Outra importante estrutura envolvida na propriocepção do sistema neuromotor é o órgão femural cordotonal ( $\mathrm{FeCO}$ - Femoral Chordotonal Organ) em insetos. Esta estrutura identifica o deslocamento linear do apódema, a qual está sujeita à deformação ocasionada pelo movimento angular da junção femorotibial do inseto. $\mathrm{O} \mathrm{FeCO}$ tem, como seu modelo análogo nos vertebrados, os fusos musculares muscle-spindles. O fuso muscular, nos vertebrados, é um mecanorreceptor formado por um conjunto de fibras musculares mais finas e mais curtas que as comuns, em torno das quais se enrola uma fibra nervosa sensível ao estiramento do músculo (Romero, 2000).

O conjunto das informações dadas por esses receptores sensoriais permite, por exemplo, movimentos dos membros, mesmo que que não se tenha exatamente a percepção espacial do meio. Um simples exercício que ilustra a propriocepção é a capacidade dos humanos de poder tocar as extremidades de um membro sem a percepção visual. A propriocepção é efetiva devido à presença de receptores específicos que são sensíveis a alterações físicas, tais como variações na angulação de uma articulação, rotação de um membro, tensão exercida sobre um músculo, e até mesmo o estiramento de uma fibra muscular.

\subsubsection{Conexões sinápticas relacionadas ao processamento local}

Uma parte do sistema nervoso, envolvida no processamento local e respostas neurais, é composta por neurônios sensores e motores que estão submetidos a um controle para gerar ações motoras, resultando na contração de um membro. Sua principal função é enviar um comando local neural, através da inervação da musculatura responsável pelas movimentos dos membros de insetos (Dewhirst et al., 2009).

Os sistemas de controle local têm como base neurofisiológica uma sequência de estímuloresposta, conhecida como arco reflexo. $\mathrm{O}$ arco reflexo é uma resposta mecânica, relativamente rápida dos membros, que em diversos casos serve como uma reação ou uma adaptação do organismo, sendo originado a partir de um estímulo. Esse tipo de processamento ocorre de forma local e é muito presente em insetos, pois estes não dependem de uma resposta direta do cérebro. Mas, prioritariamente de uma resposta local do sistema neuromotor (Burrows et al., 1988). Desta forma, quando se comparam essas respostas entre estruturas neurais de insetos 
e mamíferos, observa-se que as respostas do processamento local possuem comportamentos análogos, porém funcionalmente diferentes. No caso dos mamíferos, por exemplo, o cérebro possui papel central no processamento dos movimentos de um membro. Pois, mesmo sobre o efeito do arco reflexo, pode ser apenas uma resposta local involuntária, mas que continua sofrendo ações neurais vindas do cérebro (Devasahayam, 2013).

De forma diferente ocorre nos insetos, em que o processamento local do arco reflexo é um mecanismo que tem pouca ou quase nenhuma influência do cérebro. No entanto, a resposta local serve muito bem à sobrevivência do animal, pois a partir deste mecanismo permite-se que os membros sejam acionados para a fuga a algum predador que gere um estímulo local do sistema mecanorreceptor do inseto (Burrows, 1996). A atividade no arco reflexo tem seu início em um receptor sensorial com potencial receptor, cuja amplitude é proporcional à intensidade do estímulo. Se o estímulo for suficientemente intenso, gera-se em seguida uma ação de potencial no neurônio sensor. Os potenciais de ação dos neurônios estimulam no sistema nervoso central, potenciais sinápticos inibitórios ou excitatórios. No nervo eferente, por sua vez, são geradas os potenciais de ação que ativam um músculo.

Um reflexo é, ainda, definido como uma resposta estereotipada, isto é, padronizado a um estímulo sensorial. Ele é inato e ocorre do mesmo modo em todos os indivíduos de uma espécie. Um grande número de reflexos, de diferentes graus de complexidade, participam da regulação do meio interno, bem como da realização de movimentos padronizados de locomoção. O tipo mais simples é representado pelo reflexo rotular (patelar ou miotático), que envolve apenas dois tipos de neurônios - um sensorial, que inverva os fusos musculares do músculo quádriceps, e um neurônio motor que supre uma unidade motora dentro do mesmo músculo (Romero, 2000).

A Figura 2.5 mostra um exemplo de um circuito neural do reflexo rotular, com resposta local, envolvendo: neurônios sensores (sinais de entrada), interneurônios (processamento da informação) e neurônios motores (sinais de saída).

Assim, pode ser dito que antes mesmo de o cérebro tomar conhecimento do estímulo periférico, consequentemente, antes deste comandar uma resposta. Em mamíferos, os arcos reflexos são comandados pela substância cinzenta da medula espinhal e do bulbo. $\mathrm{O}$ arco reflexo depende de algumas sinapses, dessa região de integração, para o processamento local da informação neural (Devasahayam, 2013). Nos insetos, cada membro possui um grande grupo de neurônios sensores. São divididos entre os que atuam principalmente como proprioceptores e outros que atuam através de extereoceptores. Os extereoceptores estão relacionados à sensação 


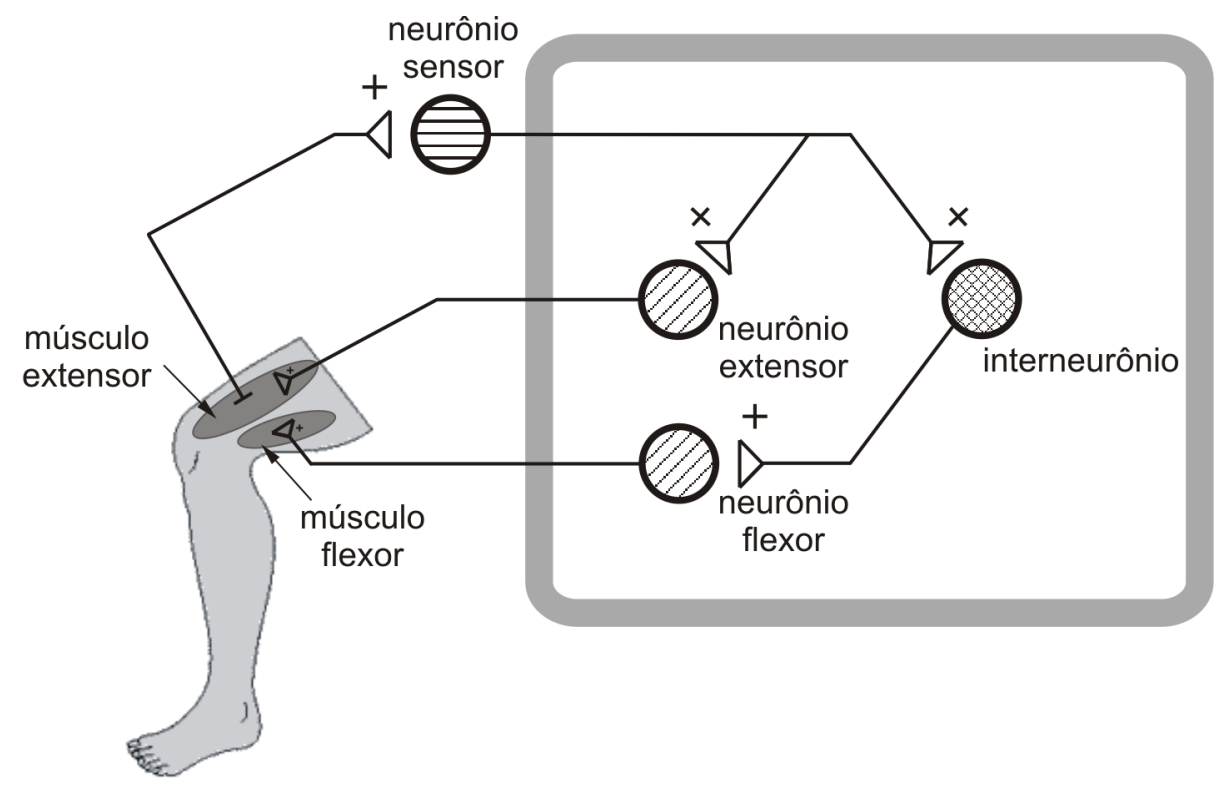

Figura 2.5: Circuito neural do reflexo local da perna. O processo envolve: neurônios sensores (sinais de entrada), interneurônios (processamento da informação) e neurônios motores (sinais de saída). [Adaptada de Byrne (1997)]

táctil do meio externo que pode ser ativado pelo deslocamento de ar provocado pela presença de algum predador. Essas estruturas sensoriais são espécies de pelos tácteis, chamadas de sensilas (Burrows, 1996).

\subsection{Neurofisiologia e estrutura do sistema nervoso central e motor de gafanhotos}

O sistema nervoso dos insetos, por exemplo, o dos gafanhotos, é distribuído e formado por uma série de segmentos ganglionares no tórax, cabeça e abdomen, os quais são responsáveis por gerar o movimento local dos membros do inseto (Burrows et al., 1988), (Burrows, 1996). Esses sistemas neurais são divididos em três partes (Snodgrass (1935) apud Burrows (1996)): o sistema nervoso somático, o sistema nervoso periférico e o sistema nervoso visceral. O sistema nervoso somático é constituído de uma cadeia ventral simétrica e bilateral de gânglios, incluindo o cérebro, que está conectado por axônios ao longo do corpo do inseto. O sistema periférico consiste nos axônios e terminações nervosas dos neurônios motores e sensores, os quais estão conectados aos gânglios do sistema nervoso somático. O sistema nervoso visceral consiste de pequenos gânglios distintos dos gânglios do sistema nervoso somático, próximos do 
cérebro ou do estômago. A Figura 2.6 mostra como é composto o sistema nervoso distribuído de um gafanhoto.

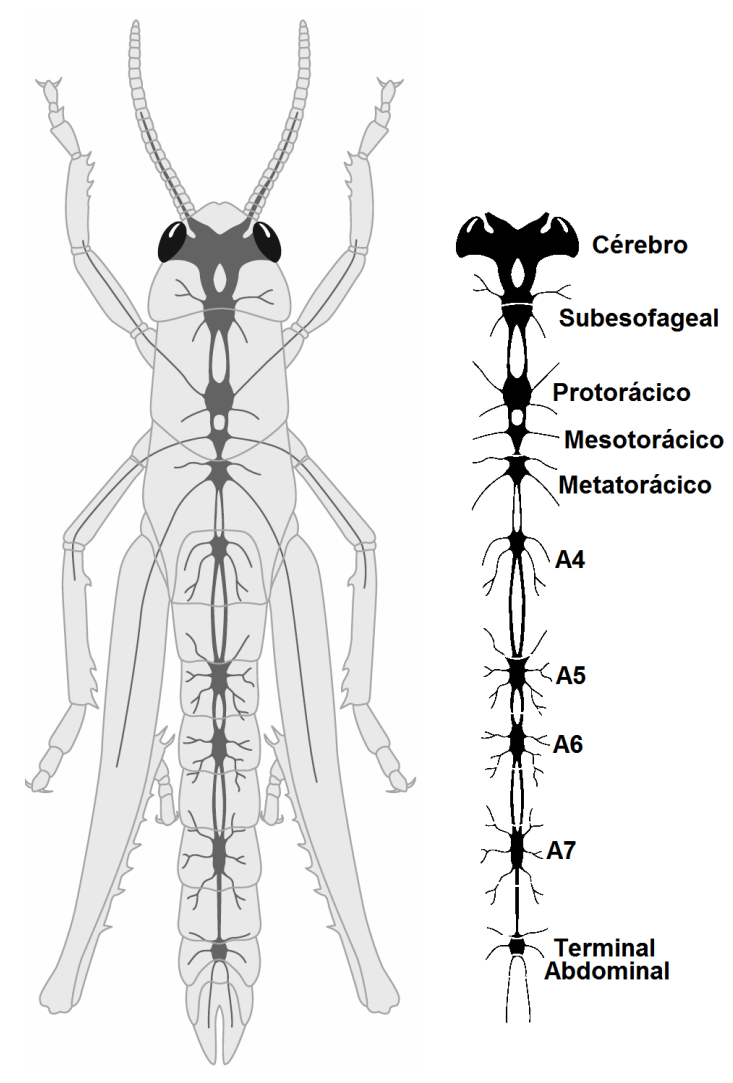

Figura 2.6: Desenho de um gafanhoto do deserto Schistocerca Gregaria e o diagrama de seu sistema nervoso em escala. [Adaptada de Pflüger e Duch (2011)]

O sistema nervoso somático de um inseto possui uma série de segmentos agrupados em gânglios, os quais são conectados entre si por terminações nervosas. Esta estrutura nervosa é composta por gânglios denominados: Mesotorácico (MESO), Metatorácico (META), Protorácico (PRO) e Subesofageal (SOG), que seguem uma distribuição ao longo do corpo do inseto de acordo com o esquemático da Figura 2.6. Esses diferentes gânglios possuem funções específicas relacionadas à anatomia local, para o controle, por exemplo, dos movimentos dos membros anteriores, intermediárias ou traseiras relacionadas à localização de cada gânglio (Pflüger e Duch, 2011). As denominações das partes da estrutura neural do gafanhoto são descritas em termos de suas relações anatômicas com outros neurônios. Isso significa que as definições e funções de um neurônio são atribuídas de acordo com sua localização, conexão com algum membro ou com as regiões sensoriais formadas por pelos tácteis (Burrows, 1996).

Todas essas estruturas são bem descritas na literatura do estudo de insetos (Burrows, 1987), (Burrows, 1996). As terminações nervosas de toda estrutura neural de inseto são caracterizadas 
levando-se em conta a combinação de anatomia, fisiologia e química celular. O entendimento da anatomia do inseto permite estudar as partes do sistema neural e motor, mapeando suas conexões físicas e estruturais. A investigação fisiológica busca relacionar as funções do sistema nervoso dos insetos com as suas respectivas ações (Burrows et al., 1988). Os movimentos motores dos membros dos insetos são acionados tanto de forma local, quanto por ação direta do cérebro do animal. Quando os movimentos são acionados localmente, estes estão relacionados aos sinais recebidos por partes sensoriais do inseto. Por exemplo, quando um inseto estiver na presença de um predador e ocorrer deslocamento do ar, as cerdas ou sensilas (pelos) tácteis identificam essas vibrações, iniciando uma sequência de ações que percorrem todo o circuito neural para a produção do movimento dos membros locomotores para a fuga do inseto (Burrows, 1996).

Os sensores receptores associados ao movimentos das pernas do inseto podem ser divididos em dois tipos: os exteroceptores e os propriceptores (Kondoh et al., 1995). A superfície do corpo do inseto é coberta por sensila's tácteis que ocorrem em diferentes densidades e tamanhos dependendo de sua localização anatômica. A essas sensila's têm-se associado os exteroceptores, pois recebem a informação sensorial externa. São basicamente de dois tipos: sensila's tricogênicas e a sensila basicogênica. As sensila's tricógenas são pelos cujos dentritos dos neurônios estão conectados à base do pelo associado a um neurônio simples. Grupos dessas sensilas são encontrados na parte posterior da cabeça, nos membros ou nas junções, informando a respeito do movimento do próprio corpo do inseto. Os sensilos basicogênicos são encontrados ao longo do corpo de inseto, incluindo os membros locomotores. E são particularmente concentrados nas antenas e em algumas partes da boca do inseto (Burrows, 1996).

Nos insetos, existem dois tipos de proprioceptores, já citados anteriormente, dos quais destacam-se: as sensila's campaniformes e o órgão cordotonal (Pflüger (1980) apud Burrows (1996)). Os sensilos campaniformes são discos ovais planos que servem para perceber qualquer flexão ocorrida no exoesqueleto. São encontrados por todo o corpo do inseto, especialmente nos membros, na base das asas e ao longo das suturas onde duas partes do exoesqueleto se encontram. Os órgãos cordotonais incluem vários tipos de proprioceptores nos quais um ou mais neurônios estabelecem ligação entre duas superfícies internas do exoesqueleto. Podendo estar relacionadas aos movimentos de um tendão ou ligamento, denominados de apódemas (Vidal-Gadea et al., 2010). O estímulo táctil desses sensores receptores provoca movimento a partir de reflexos locais nos insetos, conforme será descrito nas próximas seções. 


\subsubsection{Gânglios torácicos}

Os gânglios torácicos são encontrados em três formas, compostas pelo: protorácico, mesotorácico e metatorácico, mostrados em destaque na Figura 2.7. De todas as estruturas neurais de um inseto, essas são as que mais têm tido estudos desenvolvidos. Isso ocorre, pois estes gânglios são responsáveis pelo controle e processamento dos sinais das membros anteriores, medianos e posteriores do inseto, respectivamente. Cada parte do gânglio torácico é bilateralmente simétrico e possui cerca de 2000 neurônios cada um (Burrows, 1996).

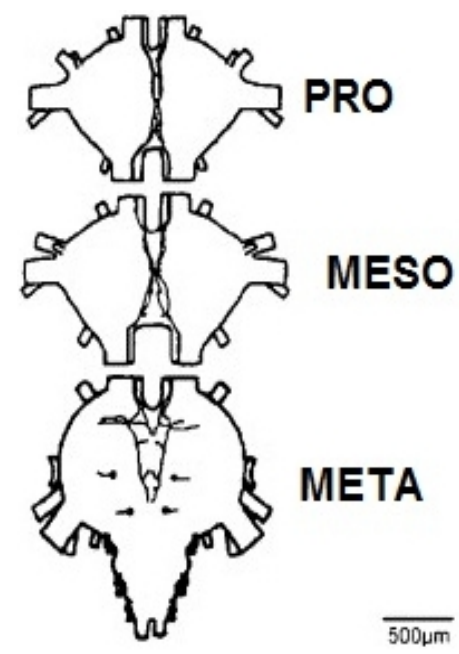

Figura 2.7: Representação do gânglio torácico de um inseto. São mostradas as três estruturas que compõem este gânglio: protorácico (PRO), mesotorácico (MESO) e metatorácico (META). [Adaptada de Pätschke et al. (2004)]

A Figura 2.8 mostra, em detalhes, os neurônios presentes no gânglio metatorácico, foco deste trabalho. Este é responsável pelo controle e processamento dos sinais do membro posterior do gafanhoto. E é uma importante parte responsável pelo controle e resposta local do membro posterior desses insetos (Dewhirst et al., 2009).

\subsubsection{Femoral Chordotonal Organ (FeCO)}

Nesta subseção é apresentada de forma detalhada o órgão cordotonal femoral, o $\mathrm{FeCO}$, responsável por uma das respostas do sistema proprioceptor de insetos. Este agrupamento de neurônios consiste de muitas células sensoras que codificam as informações do apódema. O apódema é um ligamento muscular nas junções das membros do inseto. Quando a junção femorotibial é tensionada, o apódema é esticado e excita o grupo de neurônios sensores, enquanto que na direção oposta o apódema excita um diferente grupo desses neurônios. 


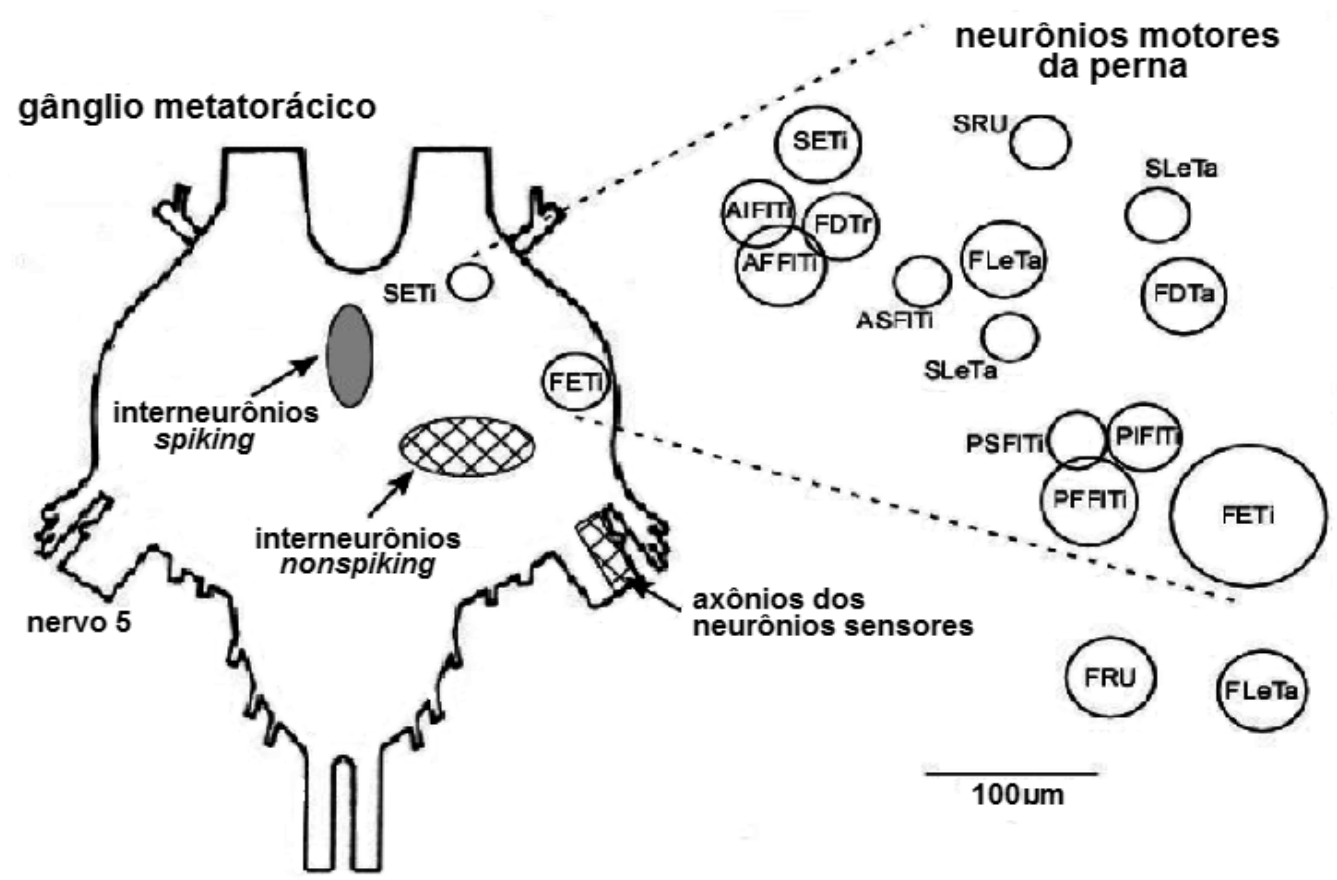

Figura 2.8: Gânglio metatorácico do sistema neuromotor local destinado ao controle dos reflexos da membro posterior do gafanhoto (vide descrição dos principais neurônios motores na Tabela 2.1). Detalhe para os locais de obtenção e coleta dos biossinais. [Adaptada de Burrows (1996)]

$\mathrm{O} \mathrm{FeCO}$ é um órgão cordotonal bastante detalhado na literatura e que está presente nos gafanhotos. Esta estrutura de neurônios sensores está presente nos membros anteriores do gafanhoto, localizada de forma distal na extremidade próxima à junção motora, conectando o apódema às fibras musculares do membro. A Figura 2.9 mostra a localização e estrutura do $\mathrm{FeCO}$. Os movimentos relativos da tíbia em relação ao fêmur do membro posterior são monitorados e codificados pelo $\mathrm{FeCO}$. Essa estrutura sensorial é composta de aproximadamento 90 células sensoriais e converte o estímulo mecânico gerado pelo apódema em sinais neurais bioelétricos (Kondoh et al., 1995).

\subsection{Caracterização dos neurônios e tipos de sinais neurais do in- seto}

Definir a função, a localização ou o tipo de sinal dos neurônios não é um problema trivial, pois, com base nos diversos estudos existentes para caracterizá-los, juntar todas essas informações em um único conceito acaba tornando muito extensa a sua descrição completa (Rowell (1989) apud Burrows (1996)). Tendo em vista essa ideia, torna-se necessária a obtenção de 


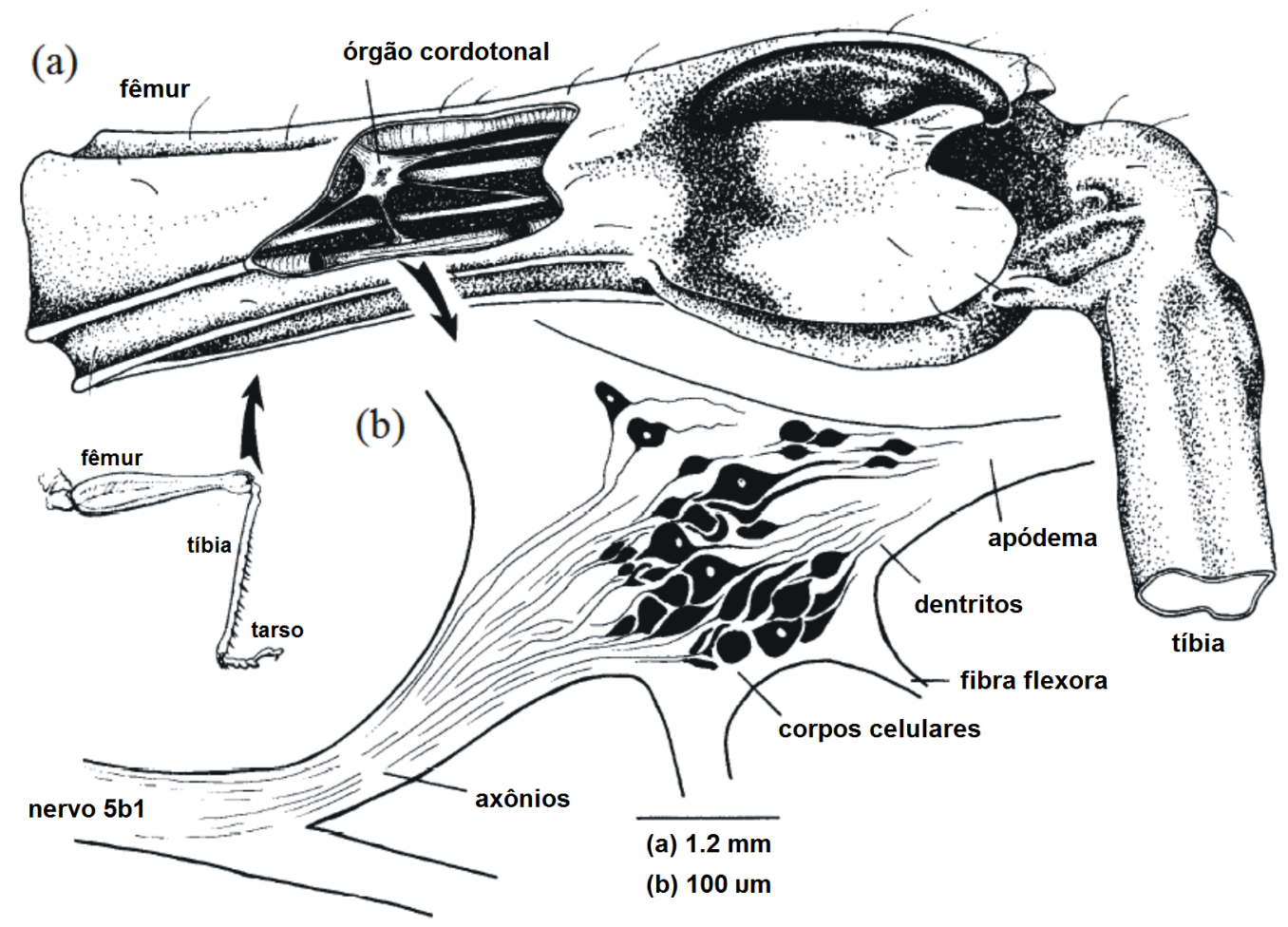

Figura 2.9: Localização e estrutura do $\mathrm{FeCO}$. (a) Região dos neurônios sensores que detectam os movimentos femorotibiais e (b) Estrutura neural do $\mathrm{FeCO}$ englobando o apódema, dentritos e o axônio. [Adaptada de Burrows (1987)]

modelos de circuitos neurais que descrevam as principais características que envolvem o processamento de sinais neurais para uma determinada função. Esses circuitos são descritos com base em um conjunto de informações sobre o processo em estudo, sem ambiguidade e com informações claras, para a identificação e os tipos dos neurônios envolvidos nos circuitos neurais (Burrows, 1996).

Os neurônios são descritos conforme sua função, topologia ou seu respectivo tipo de sinal: spiking e nonspiking. No caso dos neurônios sensores (sinais de entrada) e dos neurõnios motores (sinais de saída), eles são definidos de acordo com sua topologia e função neural, e, no caso dos interneurônios locais, são classificados de acordo com o tipo de sinal que transmitem nas conexões sinápticas.

\subsubsection{Neurônios sensores}

Os neurônios, que enviam sinais neurais do sistema nervoso sensorial para o sistema nervoso central, são chamados de aferentes. Neste caso, são representados pelos neurônios sensores, que são formados por conjuntos de dezenas de milhares de neurônios. Dos quais são 
responsáveis, por exemplo, desde a detecção de odor nos receptores das antenas, até pelos receptores da sensação táctil ao longo do corpo de animal. Incluem-se, também, os neurônios responsáveis pela percepção espacial dos membros que controlam os potenciais de ação da inibição e excitação dos músculos dos membros.

A partir da sensibilidade desses neurônios, são provocados tantos movimentos locais dos membros do inseto, quanto respostas processadas em conjunto com outras partes do sistema nervoso. Por exemplo, quando muitos pelos tácteis são estimulados, ocorre um movimento imediato do membro. No entanto, o simples toque de um único pelo seria suficiente para produzir um movimento local coordenado do membro (Pflüger (1980) apud Burrows (1996)). Outra forma de ocorrer um movimento produzido pelo arco reflexo do membro posterior do inseto é um forte estímulo causador de uma deformação na cutícula que excita os sensilos campaniformes. É difícil afirmar que, isoladamente, exteroceptores ou proprioceptores causem os movimentos locais dos membros. Pois, todos os movimentos dependem de um conjunto de situações que envolvem as interconexões entre diferentes partes do sistema nervoso do inseto (Burrows, 1996). Tipicamente, os neurônios sensores enviam sinais neurais em impulsos (spikes) (Burrows, 1996).

\subsubsection{Neurônios motores}

Os neurônios motores podem ser mais fáceis de identificar, pois estão diretamente relacionados ao músculo ou membro que inervam. Assim, é possível dizer que existe um grupo de neurônios que inervam um músculo específico e que estes números são definidos pela anatomia do animal. Os neurônios motores extensores da tíbia são aqueles que, obviamente, estão inervados naquele músculo (Burrows et al., 1988). Eles podem ser subdivididos de acordo com o tipo de contração que causam ou com a posição de suas células no sistema nervoso central (Burrows, 1996). Na Figura 2.8 e na Tabela 2.1, são destacados os neurônios motores de acordo com sua localização e sua função de estender ou flexionar um músculo da membro posterior do inseto. Na Tabela 2.1, é mostrada a função de cada neurônio motor do gânglio metatorácico, de acordo com sua topologia, função e ação.

Os sinais dos neurônios sensores são processados em redes neurais que contêm diferentes tipos de interneurônios. Esses interneurônios são responsáveis pela ativação de onze neurônios motores ( 9 causando flexão e 2 causando extensão) dos músculos da tíbia para gerar o seu movimento (Newland e Kondoh, 1997b). Os interneurônios locais possuem um largo espectro 
Tabela 2.1: Descrição dos principais neurônios motores utilizados nas coletas de sinais.

\begin{tabular}{cl}
\hline \hline \multicolumn{2}{c}{ Movimentos sobre a junção coxa-femoral } \\
\hline ASFITi & Anterior Slow Flexor tibia motor neurone \\
AFFITi & Anterior Fast Flexor tibia motor neurone \\
AIFITi & Anterior Intermediate Flexor tibia motor neurone \\
PSFITi & Posterior Slow Flexor tibia motor neurone \\
PFFITi & Posterior Fast Flexor tibia motor neurone \\
PIFITi & Posterior Intermediate Flexor tibia motor neurone \\
SETi & Slow Extensor Tibia motor neurone \\
FETi & Fast Extensor Tibia motor neurone \\
\hline \multicolumn{2}{c}{ Movimentos sobre o tornozelo } \\
\hline SleTa & Slow Levator Tarsus \\
FleTa & Fast Levator Tarsus \\
SDTa & Slow Depressor Tarsus \\
FDTa & Fast Depressor Tarsus \\
\hline \multicolumn{2}{c}{ Movimentos sobre a garra } \\
\hline SRU & Slow Retractor unguis motor neurone \\
FRU & Fast Retractor unguis motor neurone \\
\hline \multicolumn{2}{c}{ Movimentos sobre a junção corpo-trocânter } \\
\hline FLeTr & Fast Levator Trochanter motor neurone \\
FDTr & Fast Depressor Trochanter motor neurone \\
\hline \hline
\end{tabular}

de banda e podem ser classificados de acordo com seu tipo de sinal (DiCaprio, 2004), (Burrows, 1996), os quais são descritos nas próximas subseções.

\subsubsection{Interneurônios locais: spiking e nonspiking}

Todos os gânglios torácicos são formados por conjuntos de neurônios, chamados de interneurônios, responsáveis pelo processsamento da informação recebida dos neurônios sensores e transmitida a neurônios motores. A definição desses tipos de neurônios é arbitrária, mas é baseada na morfologia e no tipo de sinais transmitidos: spiking e nonspiking (Burrows, 1996). Neste trabalho, são destacados os estudos no gânglio metatorácico, por estarem relacionados ao movimento dos membros posteriores do gafanhoto.

Nas regiões dos gânglios, a definição da nomenclatura segue de acordo com sua localização e com o tipo de conexões existentes a outros conjuntos de neurônios. Assim, a definição das características dos interneurônios tem um significado funcional, por implicar que são definidos de acordo com o processamento de sinais que executam e o tipo de seus sinais: spiking e nonspiking (Burrows, 1987), (Burrows et al., 1988). Alguns desses neurônios são denominados de acordo com sua anatomia entre as partes conhecidas do inseto. As regiões de localização 
dos interneurõnios são rastreadas pelas suas inervações através da chegada ou da saída de sinais dos neurônios. De um modo geral, a identificação funcional dos interneurônios deve estar relacionada à combinação de diferentes critérios. Por isso, justifica-se a análise de seus respectivos sinais de acordo com os múltiplos caminhos percorridos no circuito neural. Esses múltiplos padrões de caminhos estão relacionados ao processamento local dos sinais entre os neurônios sensores e os neurônios motores (Pätschke et al., 2004).

O controle local dos membros é, também, originado pelos proprioceptores, os quais enviam sinais referentes aos movimentos e posições dos joelhos e monitoram a força gerada nos músculos. Os movimentos das junções, incluindo a femorotibial, são monitorados por órgãos cordotonais, entre eles o $\mathrm{FeCO}$. Alguns interneurônios spiking são dedicados somente a respostas aos proprioceptores, por exemplo, alguns interneurônios spiking. Estas respostas são os sinais de reflexo local do movimento do joelhos identificados no $\mathrm{FeCO}$ e processados pelos interneurônios locais (Dewhirst et al., 2013).

\section{Interneurônios Locais spiking}

Os interneurônios locais spiking são encontrados em todas as partes do sistema nervoso de um inseto. São mais numerosos nas inervações óticas e nos lóbulos das antenas, onde eles têm papéis distintos no processamento dos sinais dos neurônios sensores dessas regiões. Também há um grande grupo desses neurônios no gânglio torácico, e muito se conhece sobre sua morfologia e ações integrativas no processamento de sinais locais, entre neurônios sensores (exteroceptores e proprioceptores) (Burrows, 1996).

A resposta aos neurônios motores devida à ação dos neurônios exteroceptores utiliza dois grupos de interneurônios locais spiking envolvidos nessas conexões do circuito neural local. São: os interneurônios midline spiking, responsáveis pelas conexões inibitórias e os interneurônios anteromediais spiking, os quais têm a sua função relacionada às conexões de excitação dos neurônios motores, conforme pode ser visualizado pela topologia do circuito neural mostrado na Figura 2.10.

Os interneurônios anteromediais do tipo spiking, conforme visto pela conexão direta do neurônio sensor na Figura 2.10, formam um grupo excitatório de conexões entre os neurônios sensores e os neurônios motores. Não é conhecido se eles também fazem conexões com outros elementos do circuito neural, presume-se que façam. Cada interneurônio faz uma conexão divergente com alguns ou todos os neurônios motores (Newland e Kondoh, 1997b). 


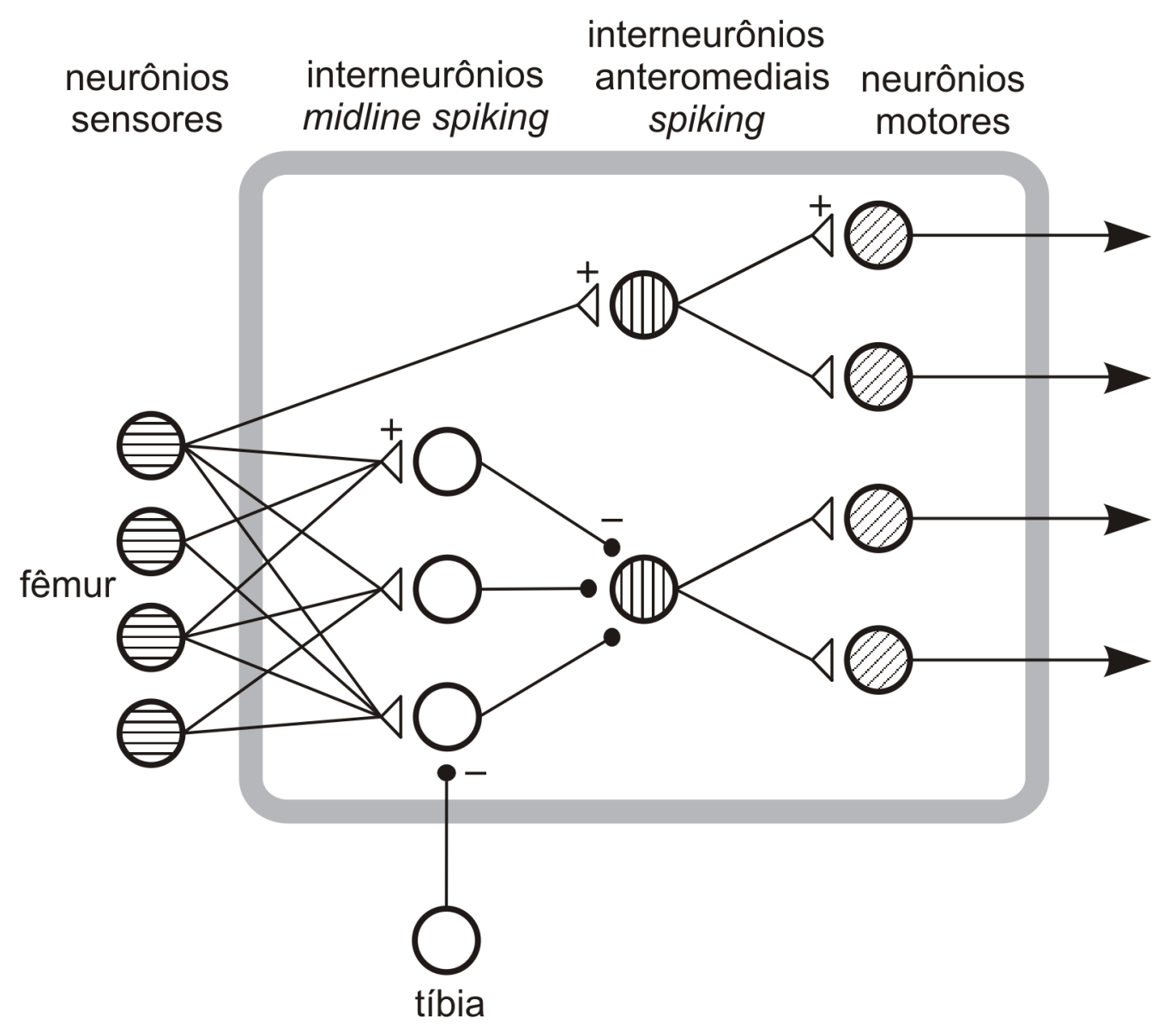

Figura 2.10: Diagrama de convergência neural no gânglio metatorácico para os interneurônios spiking que realizam a excitação dos neurônios motores gerada a partir da sensibilidade dos sensilos tricógenos recebidos através dos neurônios sensores. [Adaptada de Burrows (1996)] 
Os interneurônios spiking fazem as conexões inibitórias de saída quando estão associados com os interneurônios nonspiking conectados aos neurônios motores (Burrows, 1987), (Burrows et al., 1988). Esse arranjo de interneurônios spiking forma uma rede de conexões convergentes, feita a partir dos neurônios sensores através dos interneurônios locais spiking. A Figura 2.11 mostra o diagrama de convergência desse circuito neural e a inibição dos neurônios motores.

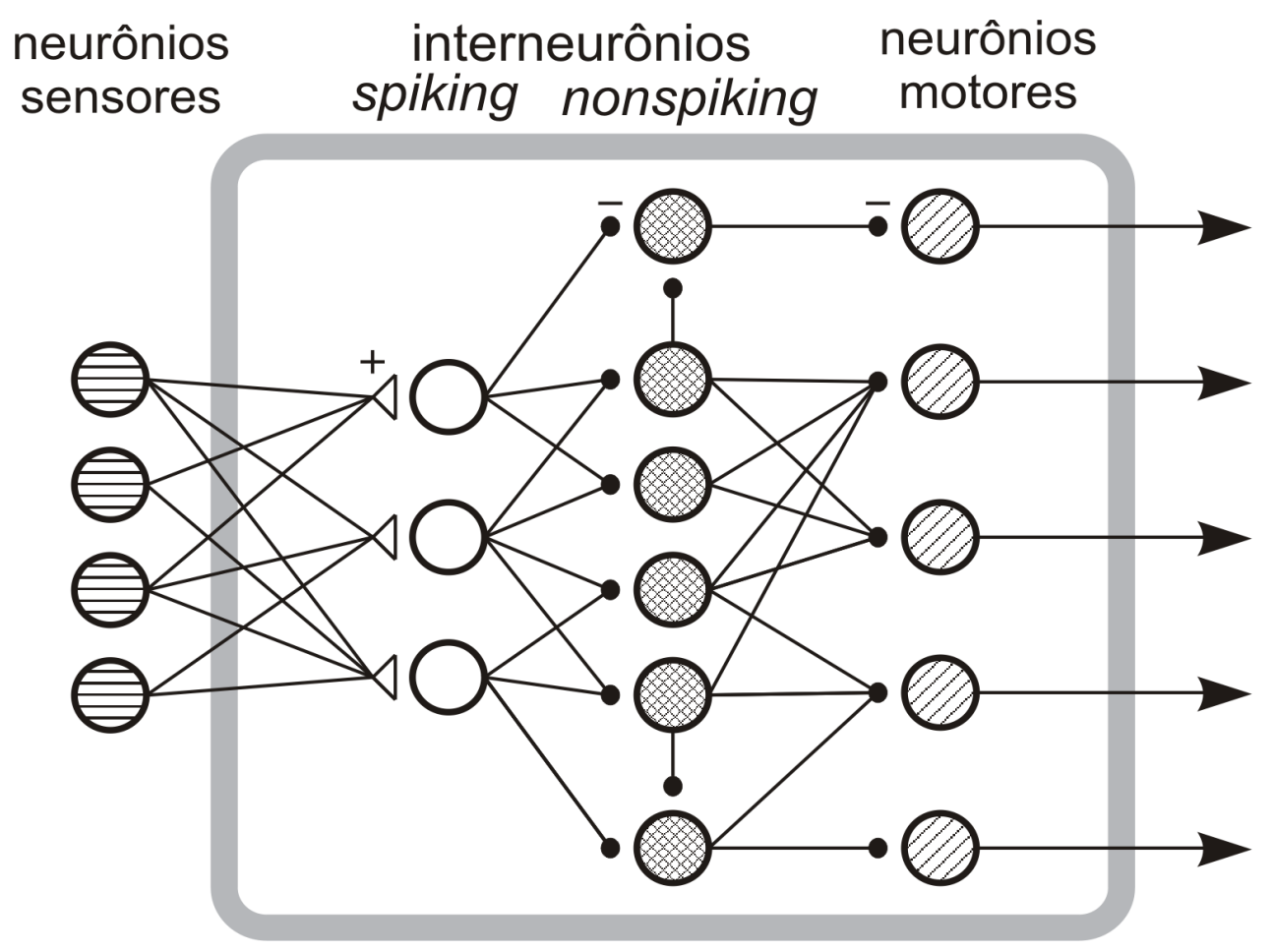

Figura 2.11: Diagrama de convergência neural do gânglio metatorácico para os interneurônios midline spiking do sistema neuromotor do membro posterior do gafanhoto. [Adaptada de Burrows (1996)]

\section{Interneurônios Locais nonspiking}

Os interneurônios locais nonspiking desempenham um importante papel na produção de reflexos locais iniciados pelos proprioceptores. Em virtude das conexões de excitação recebidas dos neurônios sensores através dos interneurônios locais spiking midline, os interneurônios nonspiking realizam as conexões de saída para os neurônios motores (Burrows et al., 1988).

O uso da palavra negativa aos interneurônios nonspiking pode ser inapropriado para descrever as diversas ações integrativas desse tipo de neurônios. Diversas pesquisas têm falhado em buscar-lhe uma nomenclatura definitiva. Assim, a denominação "nonspiking"é aplicada àqueles neurônios que não possuem a capacidade de gerar impulsos, experimentalmente definidos, 
pela depolarização direta de corrente elétrica nos axônios (Burrows, 1996). A importante característica desses neurônios, do ponto de vista da integração de sinais, é a sua habilidade em se comunicar com outros neurônios sem a presença de impulsos, através de múltiplos caminhos. E, mesmo sem a presença de spikes, exercem tanta influência sobre outras regiões do circuito neural quanto os interneurônios spiking em suas conexões pós-sinápticas (Burrows et al., 1988).

Os sinais gerados nos neurônios sensores afetam vários interneurônios locais nonspiking e, através das conexões divergentes do circuito neural, acabam gerando múltiplos caminhos que podem se sobrepor aos neurônios motores e influenciar no controle de ganho alterando a depolarização desses neurônios. Podendo resultar em um processo de excitação sináptica das entradas a partir dos neurônios sensores, ou a partir da inibição causada pelos interneurônios locais spiking (Maciel et al., 2012). 


\section{Capítulo 3}

\section{Estudos de medidas de}

\section{associação: transferência de entropia e informação.}

Neste capítulo, apresenta-se uma revisão bibliográfica dos principais conceitos utilizados neste trabalho. Inicialmente, são descritos conceitos de medidas de associação de primeira ordem entre séries temporais, destaca-se o estudo para obtenção de curvas médias de excitação para sinais neurais spiking, denominado de STA (STA-Spike Triggered Average). Foi realizado um estudo sobre as principais aplicações da transferência de informação, envolvendo: a informação mútua atrasa $(D M I)$ e a transferência de entropia $(T E)$. Outra importante ferramenta utilizada para a comparação de resultados é a técnica de Surrogate Data, pois, juntamente com a análise do nível de significância em relação aos dados originais, torna-se uma ferramenta bastante viável para validar medidas de associação entre séries temporais.

\subsection{Medidas de associação de primeira ordem entre séries tempo- rais}

As medidas de associação, em processamento de sinais e sistemas dinâmicos, são definidas como uma quantificação de similaridade ou conectividade entre dois sinais (Manolakis et al., 2005). No caso da análise de STA, a associação é medida através dos valores médios do intervalo de tempo do estímulo, anterior à ocorrência do spike (Dayan e Abbott, 2001). Outra 
medida de primeira ordem é a correlação cruzada que é obtida em função de um atraso aplicado entre os dois sinais.

\subsubsection{Estudo das curvas médias de excitação para sinais neurais spiking - Análise do Spike Triggered Average}

O STA (Spike Triggered Average) é uma ferramenta computacional para a caracterização das propriedades de resposta de um neurônio, a partir dos spikes gerados em resposta a um estímulo que varia no tempo (Dayan e Abbott, 2001). O STA fornece uma estimativa do campo receptivo linear de um neurônio, baseada nos valores médios que geram as ações de potencial que depolarizam o neurônio para a ocorrência do spike (Schwartz et al., 2006). É uma técnica bastante aplicada para a análise de dados neurofisiológicos .

A Equação 3.1 apresenta o modelo matemático do $S T A$, que é determinado através do cálculo do estímulo médio anterior a um spike. Para calcular o STA, utilizam-se os valores médios das janela de estímulo antes de cada spike.

$$
\hat{A}=\frac{1}{N} \sum_{n=1}^{N} \vec{s}\left(t_{n}\right)
$$

onde $\hat{A}$ é valor médio do estímulo e $\vec{s}$ é o vetor dos instantes de ocorrência dos spikes, sendo $\left(t_{n}\right)$ os tempos anteriores à geração dos spikes.

A Figura 3.1 apresenta um esquemático para a obtenção das janelas que são utilizadas para o cálculo da forma de onda média do estímulo que gera o spike.

O STA é conhecido como o primeiro termo no kernel das respostas dos modelos de Volterra ou Wiener (Pillow e Simoncelli, 2006). O valor encontrado pelo STA está relacionado com um modelo de regressão linear para a análise de sistemas de primeira ordem (Park e Pillow, 2011). Por outro lado, a aplicação desta técnica se torna bastante relevante nos estudos com sinais neurofisiológicos em que se deseja obter a relação linear de um estímulo com a geração de spikes, tal como em sinais neurais (Dayan e Abbott, 2001). Sendo, também, utilizado para estimar a fase linear de um filtro casado, baseado em um modelo LNP (linear-nonlinear-Poisson, como pode ser visto em Pillow e Simoncelli (2006), Manolakis et al. (2005), dentre outros. Na literatura, este método para análise do estímulo médio, também é conhecido como "análise de correlação inversa"ou "análise de ruído branco"(Dayan e Abbott, 2001). O STA tem uma larga aplicação para a caracterização de células ganglionares óticas (Schwartz et al., 2006). 


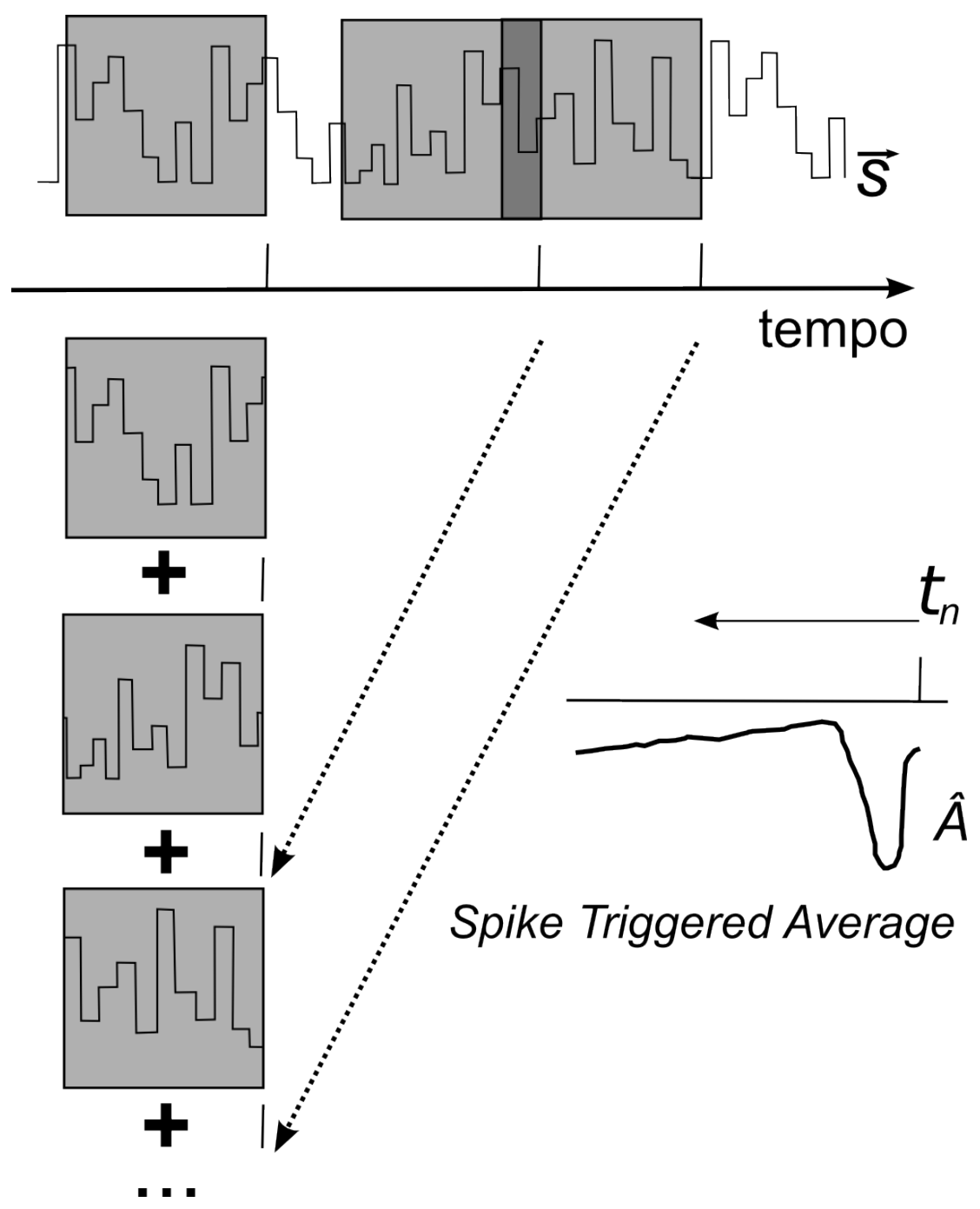

Figura 3.1: Esquemático com o procedimento para cálculo do STA. Cada retângulo contém o estímulo a priori para a ocorrência de um spike, mostrado ao longo do eixo de tempo. A partir destas janelas são calculadas a forma de onda média do estímulo, mostrada na parte direita inferior. [Adaptada de Dayan e Abbott (2001)] 
A partir da análise de STA são estimadas as funções densidade de probabilidade ( $p d f$ ) para determinar as dependências estatísticas entre a ocorrência de spikes, dada a curva de estímulo que o gerou (Schwartz et al., 2006). Ao se obter as estimativas das $p d f$ 's do sinal de estímulo, $p($ stim $)$, determina-se a função densidade de probabilidade condicional, $p($ stim $\mid$ spike $)$. Desta forma, podem ser calculadas as condições de dependências estatísticas entre estímulo e a geração de um spike. Em diversos casos, o estímulo é um ruído branco gaussiano que define a função de estímulo (Park e Pillow, 2011). Uma importante consequência, utilizando uma inferência bayesiana, é encontrar a relação condicional que define a probabilidade de ocorrênica de um spike, quando se conhece a curva de estímulo, p(spike|stim) Endo et al. (2013a). Na Equação 3.2 é descrito o procedimento para esta análise:

$$
p(\text { spike } \mid \text { stim })=\alpha \frac{p(\text { stim } \mid \text { spike })}{p(\text { stim })}
$$

onde $\alpha$ é uma constante proporcional à probabilidade de ocorrência de spikes dada como informação a priori, $p($ spike $)$, relacinada à curva de estímulo, $p($ stim $)$. E, dada a probabilidade condicional de verosimilhança, $p($ stim $\mid$ spike $)$, obtém-se pela inferência bayesiana a informação a posteriori, $p$ (spike|stim). Verifica-se na Equação 3.2, que o numerador na fração é a correlação de entrada que associa o efeito do sinal a priori ao se relacionar o estímulo na ocorrência de spikes (Park e Pillow, 2011).

\subsubsection{Correlação cruzada}

A correlação cruzada é uma medida para se quantificar o nível de associação linear entre dois sinais, $x$ e $y$, em função de um atraso aplicado entre eles (Manolakis et al., 2005). Também é denominada como produto interno deslocado. A correlação cruzada é frequentemente utilizada quando se deseja procurar por um sinal de curta duração que esteja inserido em um sinal mais longo. Por ser uma ferramenta de fácil implementação matemática, encontra diversas aplicações em reconhecimento de padrões, análise de partícula única, criptoanálise e neurofisiologia (Devasahayam, 2013).

O modelo matemático que descreve a correlação cruzada entre duas variáveis utiliza a covariância (Vaseghi, 2006), mostrada na Equação 3.3:

$$
\operatorname{Cov}=\frac{1}{N} \sum_{i=1}^{N}\left(x_{i}-\bar{x}\right) \cdot\left(y_{i}-\bar{y}\right)
$$


onde $\bar{x}$ e $\bar{y}$ são os valores médios de $x$ e $y$, respectivamente.

A equação anterior mede o quanto dois sinais variam conjuntamente. A correlação entre duas variáveis é a covariância (Cov) de dois sinais dividido pelo produto dos desvios padrão (Vaseghi, 2006). A correlação cruzada é normalizada no intervalo de -1 a +1 , conforme mostrado na Equação 3.4:

$$
\operatorname{Corr}(x, y)=\frac{\operatorname{Cov}(x, y)}{\sqrt{\operatorname{Var}(x) \cdot \operatorname{Var}(y)}}
$$

onde Var é a variância.

A correlação cruzada (Corr) possui certa semelhança com a convolução de duas funções (Manolakis et al., 2005). Porém, ao contrário da convolução, na correlação cruzada não há espelhamento de um dos sinais. Outra importante propriedade que distingue essas duas operações é que a convolução é comutativa, o que não ocorre na correlação cruzada (Diniz et al., 2004).

$\mathrm{Na}$ aplicação em circuitos neurais, a correlação cruzada pode ser um ferramenta computacional pobre quando se tem associações que extrapolem modelos puramente lineares, entre o estímulo e os sinais neurofisiológicos em estudo (Vicente et al., 2011). Pois, em muitos tipos desses sistemas o que ocorre é a sobreposição de componentes lineares e não-lineares no modelo de associação entre os sinais (Yang et al., 2013). Para esses casos, é necessária a utilização de ferramentas mais robustas que obtenham esses tipos de medidas de associação, por exemplo: a informação mútua e a transferência de entropia.

\subsection{Entropia e informação mútua como medidas de associação}

Utilizando-se a entropia de Shannon entre duas variáveis, $H(X)$ e $H(Y)$, é possível determinar o quanto de informação é compartilhada, entre si. Define-se que a informação mútua entre $X$ e $Y, I(X, Y)$ quantifica a informação compartilhada com base nas medidas de incertezas dadas pelas entropias condicionais dessas duas variáveis. Uma outra forma de interpretação é o quanto de conhecimento sobre uma série informa sobre a outra (Cover e Thomas, 2006). Esta equação é descrita em diversas referências, em termos das suas respectivas entropias, como em (Cover e Thomas, 2006; Shannon, 1948): 


$$
\begin{aligned}
I(X ; Y) & =H(Y)-H(Y \mid X) \\
& =H(X)+H(Y)-H(X ; Y)
\end{aligned}
$$

onde $H(Y \mid X)$ é a entropia condicional de $Y$ dado $X$. Sendo $H(Y) \geq H(Y \mid X)$ então $0 \leq$ $I(X, Y)<\infty$ (Dionisio et al., 2004), a igualdade é dada, se, e somente se, $X$ e $Y$ forem variáveis independentes.

Na Figura 3.2 é mostrada, através de um diagrama de Venn, a ideia intuitiva que ilustra o que é definido como informação mútua entre duas variáveis

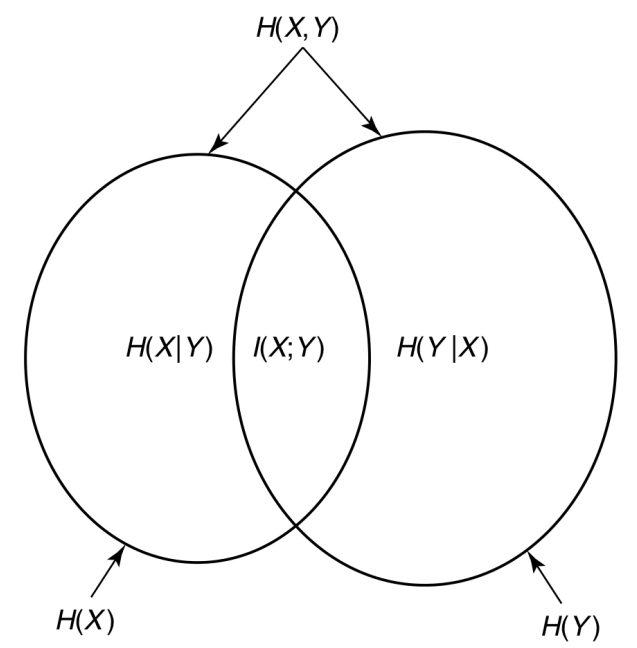

Figura 3.2: Relação de entropia entre duas variáveis aleatórias. É representado o significado da informação mútua e das entropias condicionais pela teoria de conjuntos. [Fonte: Cover e Thomas (2006)]

A informação mútua é aplicada como uma medida de associação entre séries temporais (Dionisio et al., 2004). As medidas de associação ou conectividade são avaliadas pela descrição conjunta de séries temporais coletadas simultaneamente a partir do sistema em estudo (Faes et al., 2012). Existem, basicamente, três tipos de medidas de associação em sistemas neurais: a associação anatômica, a associação funcional e a associação efetiva (Sporns (2004) apud Liu e Aviyente (2012)). A associação anatômica é caracterizada por conexões identificadas estruturalmente, nas quais são avaliadas as ligações entre neurônio, que é o que ocorre nas análises de conectomas cerebrais (Sporns, 2011). A associação funcional está relacionada à captura das dependências estatísticas e de acordo com as funções de um circuito neural em um sistema. A associação efetiva em circuito neurais refere-se à influência que um sistema neural 
exerce sobre outro e sua relação através de parâmetros de um modelo que busca explicar as dependências observadas (Liu e Aviyente, 2012).

Para a aplicação em circuitos neurais, um dos principais objetivos é quantificar essas medidas de associação entre os sinais. Assim, utiliza-se uma das principais consequências da entropia de Shannon, denominada de informação mútua (MI - Mutual Information).

O cálculo da $M I$ utiliza as funções densidades de probabilidades dos sinais em estudo, e é descrito pela seguinte Equação 3.7 (Cover e Thomas, 2006),

$$
I(X ; Y)=\sum_{x \in \chi} \sum_{y \in \gamma} p(x, y) \log _{2} \frac{p(x, y)}{p(x) \cdot p(y)} .
$$

onde $p(x)$ e $p(y)$ são as probabilidades marginais de $X$ e $Y$, respectivamente, tendo $p(x, y)$ a distribuição da probabilidade conjunta dessas duas variáveis.

Pode-se notar que a $M I$ depende explicitamente das probabilidades conjuntas entre $X$ e $Y$. Ao se obter, pela Equação 3.7, o valor da informação compartilhada entre as duas variáveis, mede-se o nível de associação entre essas séries temporais.

As medidas de associação obtidas pela $M I$ não dependem da identificação de um modelo ou de uma função de transferência; estas relações são extraídas diretamente dos sinais $X \mathrm{e}$ $Y$. Por não dependerem de um modelo explícito, as aplicações com a informação mútua são denominadas como model-free. Em diversas aplicações, tanto em neurociência como em outras áreas de conhecimento, a obtenção de modelos e funções de transferência não é fácil de se alcançar (Vicente et al., 2011). Como é caso de modelos com relações não lineares (Jin et al., 2010). Assim, a utilização da $M I$, nesses casos, tem boa vantagem para a obtenção de relações de causalidade sem a necessidade de um modelo explícito.

\subsection{Transferência de informação}

Transmitir dados, codificar informações e enviá-las de uma fonte a um receptor envolve o conceito de transferência de informação em sistemas de comunicação (Cover e Thomas, 2006). Aplicar esses conceitos em circuitos neurais é bastante viável, devido à sua similaridade funcional em codificar e transmitir um sinal.

Em circuitos neurais a direção de transferência de informação não é uma tarefa fácil, pois não existem protocolos de comunicação formais que definem quando o circuito recebe ou envia 
sinais. Assim, um dos principais desafios em circuitos neurais é obter a direção da transferência de informação. Em Jin et al. (2010), é apresentada a aplicação para determinação da direção da transferência de informação utilizando-se a informação mútua. Uma recente abordagem é a transferência de entropia de Schreiber (2000), que quantifica a transferência de informação bidirecional, tanto de $X \rightarrow Y$ quanto $Y \rightarrow X$, em um sistema.

\subsubsection{Atraso da informação mútua}

Nichols (2006), Alonso et al. (2007) e outros autores examinam a estrutura dinâmica de um sistema complexo, com base no atraso $y(t-\tau)$ em relação a $x(t)$ da informação mútua. A informação mútua atrasada (DMI - Delayed mutual information) quantifica a dependência entre variáveis aleatórias buscando obter a informação compartilhada em função do deslocamento do tempo, $\tau$ entre $X$ e $Y^{\tau}$. Assumindo que $X$ e $Y$ são sinais estacionários e que a probabilidade conjunta dependerá apenas do tempo de atraso $\tau$, a equação que descreve a DMI, de acordo com Nichols (2006), é:

$$
I\left(X ; Y^{\tau}\right)=\sum_{x_{n} \in \chi} \sum_{y_{n-\tau} \in \gamma} p\left(x_{n}, y_{n-\tau}\right) \log _{2} \frac{p\left(x_{n}, y_{n-\tau}\right)}{p\left(x_{n}\right) p\left(y_{n-\tau}\right)} .
$$

onde $X$ e $Y$ podem ser definidos como processos estocásticos, sendo $X=\left(x_{1}, x_{2}, \ldots, x_{n}\right)$ e $Y^{\tau}=\left(y_{1-\tau}, y_{2-\tau}, \ldots, y_{n-\tau}\right)$ variáveis aleatórias. $p\left(x_{n}\right)$ e $p\left(y_{n-\tau}\right)$ são as funções densidade de probabilidade $(p d f)$ marginais de $X$ e $Y^{\tau}$, respectivamente. A $p d f$ conjunta é dada por $p\left(x_{n}, y_{n-\tau}\right)$, a qual é uma função do tempo de atraso, $\tau$.

Ao se aplicar a DMI entre séries temporais, é discutida a ocorrência da sincronização de fases através do atraso da informação (Yang et al., 2013), (Silchenko et al., 2010). As relações de causalidade são identificadas através dessa medida baseada no atraso, $\tau$. $\mathrm{O}$ atraso com base na informação é obtido quando ocorrem picos de máxima coerência (Silchenko et al., 2010). A obtenção desses picos de máxima coerência depende diretamente da identificação da diferença de tempo e da máxima $M I$ entre duas séries temporais coletadas simultaneamente (Wilmer et al., 2012). Um problema interessante de tempos de atraso é apresentado em Endo et al. (2013a), para aplicação em arranjos de múltiplo sensores e que envolve medidas de coerênca de informação.

A interpretação da curva de atraso da informação mútua considera o compartilhamento de informação enre $x_{n}$ e $y_{n-\tau}$. E a existência de um pico de máxima coerência na curva, $I\left(X, Y^{\tau}\right)$ 
para $\tau>0$, indica a concentração de informação dada pela densidade de probabilidade conjunta entre $X$ e $Y^{\tau}$, conforme pode ser visto na Figura 3.3. Caso contrário, se o pico ocorrer para $\tau<0$, implica que a informação é transferida de $Y$ para $X$ (Jin et al., 2010).

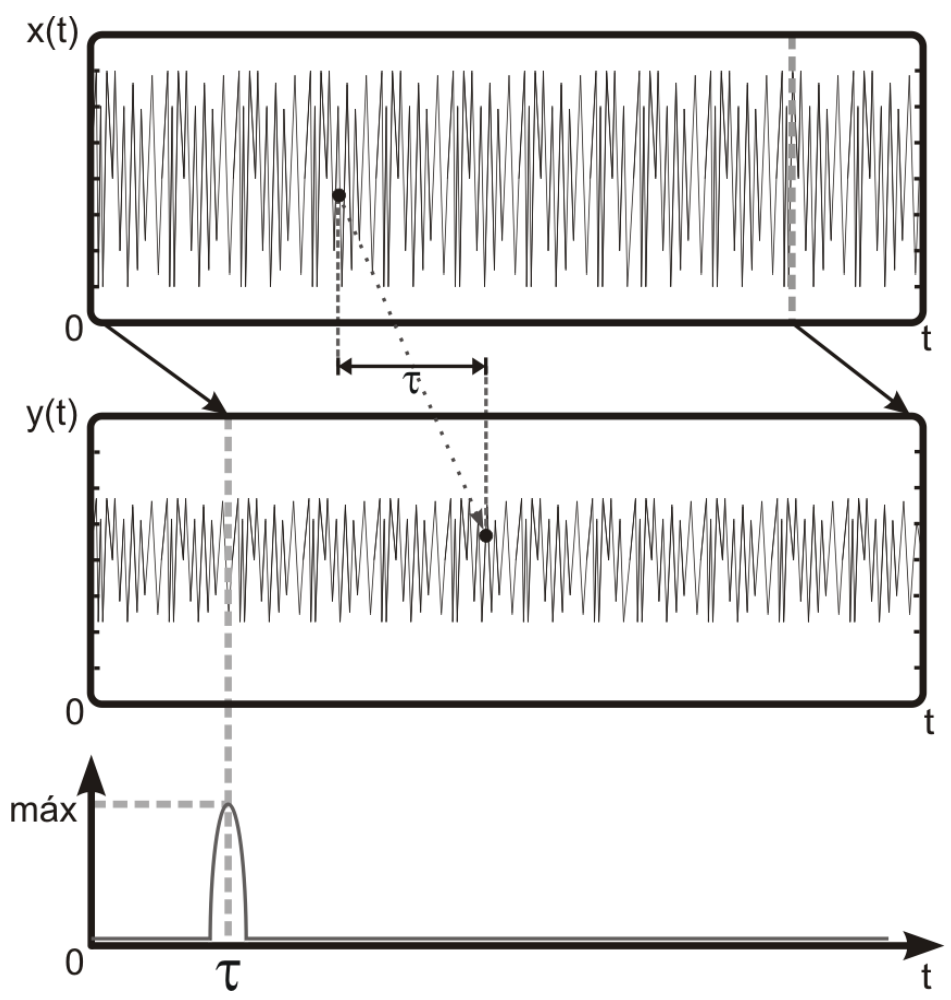

Figura 3.3: Modelo da transferência de informação entre séries temporais. Observa-se a curva de máxima coerência de informação, com um atraso, $\tau$.

No entanto, quando duas séries temporais possuem medidas bidirecionais de associação, a DMI falha em distinguir a direção dessas relações (Yang et al., 2013). Na Figura 3.4, são mostrados três padrões de relações causais. Na Figura 3.4(a), a DMI identifica a relação indireta de associação entre $X_{1}$ e $X_{3}$, a partir dos tempos de atraso e dos picos de máxima coerência. Nesse caso, a DMI tem grande sucesso, pois a informação é unidirecional e pode ser identificada mesmo de forma indireta entre $X_{1}$ e $X_{3}$. No exemplo da Figura 3.4(b), o resultado da $D M I$ já apresenta problemas, pois o resultado dos tempos de atraso e o de máxima coerência são os mesmos identificados no primeiro caso, uma vez que a bidirecionalidade é ignorada pela $D M I$. No terceiro caso, mostrado na Figura 3.4(c), existe uma associação direta entre $X_{1}$ e $X_{3}$. Este seria o pior caso para a $D M I$, pois seriam identificados dois tempos de atraso em $X 3$, sem que possa ser definido exatamente de onde vieram as informações (Wilmer et al., 2012).

No exemplo mostrado na Figura 3.4, a direcionalidade da associação de informação é difícil de se detectar. E esse caso tipicamente ocorre no gânglio metatorácico de insetos, em que 


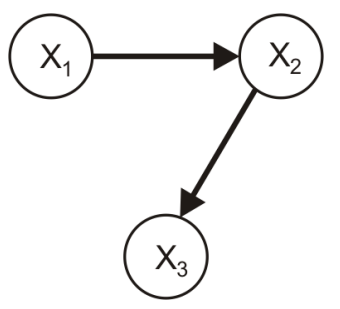

(a)

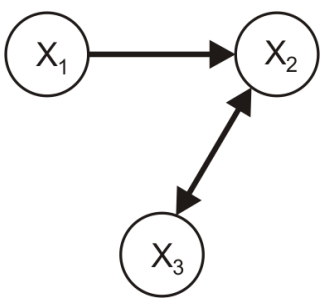

(b)

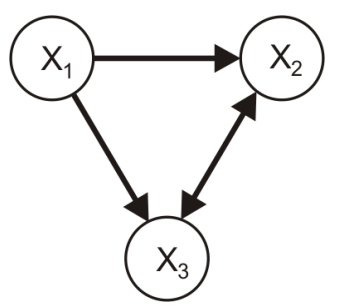

(c)

Figura 3.4: Padrões de relações causais com (a) identificação da relação indireta de associação entre $X_{1}$ e $X_{3}$; (b) ocorrência da birecionalidade, ignorada pela $D M I$; e (c) pior caso para a $D M I$, são identificados dois tempos de atraso em $X 3$, sem a definição exata da direção do fluxo de informações. [Adaptada de Yang et al. (2013)]

não é possível afirmar a existência de apenas uma direção única de associação. Somente em casos específicos de circuitos neurais, sabe-se que existe uma única direção de transferência de informação, como ocorre, por exemplo, no caso dos neurônios motores, nos quais cada neurônio inerva diretamente um músculo específico (Vidal-Gadea et al., 2010) ou como em alguns casos de nervos aferentes sensores, por exemplo $\mathrm{FeCO}$.

Ao se analisar a Equação 3.8, verifica-se que isso é consequência das suas condições de simetria, pois mesmo ao se identificar o atraso $\tau$, a $D M I$ é igual em $I\left(X, Y^{\tau}\right)$ e $I\left(Y^{\tau}, X\right)$. A ausência de uma assimetria dessa medida de associação dificulta a obtenção da conectividade em casos de transferência de informação bidirecional entre séries temporais (Schreiber, 2000).

A partir disso, são apresentados na próxima subseção os conceitos da transferência de entropia, em que são consideradas as medidas de associção para sistemas com bidirecionalidade na transferência de informação.

\subsubsection{Transferência de entropia}

A transferência de entropia (TE), de acordo com Nichols et al. (2005), determina a direção da transferência de informação, a partir das condições de assimetria entre as interações dos sistemas, $X$ e $Y$. Em Schreiber (2000), é introduzida a estrutura dinâmica de transferência de entropia, ao se analisar as probabilidades de transição. Na $D M I$, a relação dinâmica é obtida apenas pela variação do atraso $\tau$, enquanto que na $T E$ essa relação aparece com a probabilidade condicionada ao histórico comum dado por $Y_{k}^{\tau}$. Dessa forma, a interpretação da transferência de entropia é obtida da informação mútua condicional (CMI - Conditional Mutual Information) entre $X$ e $Y^{\tau}$ dado $Y_{k}^{\tau}$ (Gourévitch e Eggermont, 2007). A equação é escrita como: 


$$
\begin{aligned}
\mathrm{TE}(X \rightarrow Y) & =I\left(Y^{\tau} ; X \mid Y_{k}^{\tau}\right) \\
& =H\left(Y^{\tau} \mid Y_{k}^{\tau}\right)-H\left(Y^{\tau} \mid X, Y_{k}^{\tau}\right)
\end{aligned}
$$

onde $\tau$ é o tempo de atraso e $k$ é o tamanho do bloco.

Schreiber (2000) define a transferência de entropia como uma generalização da divergência de Kullback-Leibler $(K L)$, que mede o excesso de bits codificados, quando se troca a distribuição de probabilidade $p(x)$ por uma distribuição $q(x)$, para codificar uma mesma série temporal. Dessa forma, a divergência de Kullback-Leibler é definida pelas probabilidades condicionais $p(x \mid y)$, conforme é mostrado na Equação 3.10:

$$
K L_{X \mid Y}=\sum_{x, y} p(x, y) \cdot \log \frac{p(x \mid y)}{q(x \mid y)}
$$

No entanto, a divergência de Kullback-Leibler ainda possui condições de simetria sobre a transferência de informação de $X \rightarrow Y$. Por isso, utiliza-se uma medida de probabilidades condicionais baseada na propriedade generalizada de Markov (Schreiber, 2000).

$$
p\left(x_{n+1} \mid x_{n}^{(k)}\right)=p\left(x_{n+1} \mid x_{n}^{(k)}, y_{n}^{(k)}\right)
$$

Na ausência de transferência de informação de $Y$ para $X$, o estado de $Y$ não tem influência na transmissão de probabilidades no sistema $X$. Assim, esses valores podem ser obtidos pela reinterpretação da divergência de Kullback-Leibler, na qual é definida a Transferência de Entropia de $Y \rightarrow X$ (Schreiber, 2000):

$$
T E_{Y \rightarrow X}=\sum_{x, y} p\left(x_{n+1}, x_{n}^{(k)}, y_{n}^{(k)}\right) \cdot \log \frac{p\left(x_{n+1} \mid x_{n}^{(k)}, y_{n}^{(k)}\right)}{p\left(x_{n+1} \mid x_{n}^{(k)}\right)}
$$

De forma análoga, a $T E_{X \rightarrow Y}$ é reescrita como:

$$
T E_{X \rightarrow Y}=\sum_{x, y} p\left(y_{n+1}, y_{n}^{(k)}, x_{n}^{(k)}\right) \cdot \log \frac{p\left(y_{n+1} \mid y_{n}^{(k)}, x_{n}^{(k)}\right)}{p\left(y_{n+1} \mid y_{n}^{(k)}\right)}
$$

As Equações 3.12 e 3.13 tornam possível a identificação bidirecional da transferência de informação, pois a $T E_{X \rightarrow Y}$ é diferente de $T E_{Y \rightarrow X}$.

Alguns testes preliminares da transferência de entropia são apresentados em Schreiber 
(2000), onde é avaliado o fluxo de informação do sistema cardiorrespiratório de seres humanos em repouso, entre os batimentos do coração e a taxa de respiração. Em Kaiser e Schreiber (2002), a TE é expandida para sistema contínuos, e novos ensaios e testes são feitos para processos gaussianos. Lindner et al. (2011) apresentam uma ferramenta em código aberto para o cálculo da transferência de entropia para um toolbox em MatLab, incluindo testes em dados eletrofisiológicos e aplicados à neurociência.

\subsection{Utililização de Surrogate Data e medidas do nível de significân- cia}

Ao se aplicar medidas de transferência de informação entre séries temporais, o valor de máxima coerência ocorre a partir da sincronização de fases (Yang et al., 2013). Uma abordagem bastante aplicada é substituir uma das séries temporais reais por dados simulados e verificar se, a partir dos dados substitutos, o valor de máxima coerência se mantém. Essa técnica de substituição de dados é denominada Surrogate Data (Schreiber e Schmitz, 2000).

A geração desses dados simulados segue algumas regras como referência para a medição do nível de significância em relação aos dados originais. O conceito da Surrogate data baseia-se em estimar as estatísticas não lineares para o conjunto de dados originais a serem introduzidas para os novos dados de simulação (Dolan e Spano, 2001). Diversas técnicas são utilizadas para gerar o Surrogate data para a análise estatística.

Um método bastante utilizado é baseado na Transformada de Fourier. Nesse procedimento são embaralhadas as fases dos sinais e, em seguida, são gerados os dados simulados através da transformada inversa (Schreiber e Schmitz, 1996). Um algoritmo para a geração desses dados simulados é apresentado em Schreiber e Schmitz (2000), chamado de IAFFT (Iterative Amplitude Adjusted Fourier Transform). Uma discussão sobre essa técnica também está disponível em Dolan e Spano (2001). Esse algoritmo gera o Surrogate data, com a mesma amplitude e a mesma distribuição da densidade espectral de potência (Venema et al., 2006).

Para a verificação dos testes estatísticos, aplica-se a comparação entre as medidas de transferência de informação (DMI ou TE) obtidas com os dados reais e com a geração do Surrogate Data. A medição do nível de significância $S$ definido por (Jin et al., 2010): 


$$
S=\frac{\left|\left\langle\Upsilon_{\text {surro }}\right\rangle-\Upsilon\right|}{\sigma\left(\Upsilon_{\text {surro }}\right)}
$$

onde $\Upsilon$ é definido pela $D M I$ ou $T E ;\left\langle\Upsilon_{\text {surro }}\right\rangle$ é o valor médio de $\Upsilon$ obtido a partir do Surrogate Data; e $\sigma\left(\Upsilon_{\text {surro }}\right)$ o desvio-padrão dessa medida de associação.

Assim, se as estatísticas obtidas a partir dos dados reais forem significativamente diferentes dos valores obtidos para os dados substitutos, infere-se que os Surrogate data foram gerados por processos aleatórios descorrelacionados, validando, dessa forma, a medida de associação ocorrida a partir dos dados reais (Schreiber e Schmitz, 2000).

\subsection{Normalização das medidas de transferência de informação}

Utilizar valores absolutos para estudos que envolvem comparação de dados entre si é um problema, pois diferentes amostras de dados estão sujeitas a vários erros de desvio (erros sistemáticos). Esses erros ocorrem desde a coleta dos dados pela presença de ruídos nas linhas de base do sinal inclusive pelo tamanhos das amostras utilizadas na análise (Ince et al., 2012). Por essas razões, é necessária a aplicação de medidas normalizadas para a comparação de diferentes amostras.

As medidas de transferência de informação, $D M I$ e $T E$ ficam sujeitas a erros de desvio quando seus valores se aproximam de zero. Por exemplo, se duas séries temporais são descorrelacionadas, o valor esperado das suas medidas de associação deve ser igual a zero (Lüdtke et al., 2008). No entanto, em alguns casos, isso não ocorre, devido aos erros originados pelas estimativas estatísticas (Strehl e Ghosh, 2003). Esses erros são ocasionados tanto pela presença de ruídos brancos na coleta dos sinais, quanto por amostras de dados de tamanhos diferentes. Os trechos selecionados devem ser estacionários no sentido amplo (WSS - Wide Stationary Sense) (Diniz et al., 2002). No entanto, as séries temporais possuem tamanhos variados, alterando, assim, a característica das funções densidade de probabilidade (Ince et al., 2012). Para descartar que esses resultados sejam apenas flutuações ocasionadas pelo tamanho limitado das amostras ou pela presença de ruídos nos sinais, torna-se necessário normalizar e compensar suas medidas.

Com base em Strehl e Ghosh (2003), a DMI é normalizada pela média geométrica das entropias totais do sistema, $H(X)$ e $H\left(Y^{\tau}\right)$. A normalização da informação mútua atrasada, $\hat{I}\left(X ; Y^{\tau}\right)$, é apresentada na Equação 3.15. 


$$
\widehat{I}\left(X ; Y^{\tau}\right)=\frac{I\left(X ; Y^{\tau}\right)}{\sqrt{H(X) \cdot H\left(Y^{\tau}\right)}}
$$

Em seguida, para se compensar os erros de desvio, utilizam-se os valores médios da linha de base da curva de atraso da informação. Uma ferramenta para se obter esses valores é a utilização dos resultados a partir do Surrogate Data. Assim, estimando-se o valor médio da linha de base para a $D M I$, dado por $I_{B}\left(X ; Y^{\tau}\right)$, obtêm-se os valores de hipótese nula para a compensação dos erros de desvio. Esse termo é incluso no estimador normalizado, $\hat{I}_{B}\left(X ; Y^{\tau}\right)$, apresentado na Equação 3.16.

$$
\widehat{I}_{B}\left(X ; Y^{\tau}\right)=\frac{I\left(X ; Y^{\tau}\right)-I_{B}\left(X ; Y^{\tau}\right)}{\sqrt{H(X) \cdot H\left(Y^{\tau}\right)}}
$$

Da mesma forma, os procedimentos de normalização e compensação dos erros de desvio são aplicados à $T E$. Define-se esse estimador normalizado como $\widehat{T E}$. A principal diferença deste estimador, em relação à $\hat{I}\left(X ; Y^{\tau}\right)$, são as entropias totais do sistema, que neste caso dependem das entropias condicionais, $H\left(Y^{\tau} \mid Y_{k}^{\tau}\right)$ e $H\left(X \mid Y_{k}^{\tau}\right)$ (Gourévitch e Eggermont, 2007).

Utiliza-se, então, o estimador dos erros de desvio da transferência de entropia, $T E_{B}(X \rightarrow$ Y). Assim, a partir da normalização dada pela relação condicional das entropias totais do sistema, obtém-se a Equação 3.17 com a compensação dos erros de desvio e a sua respectiva normalização (Besson et al., 2010).

$$
\widehat{T E}_{B}(X \rightarrow Y)=\frac{T E(X \rightarrow Y)-T E_{B}(X \rightarrow Y)}{\sqrt{H\left(Y^{\tau} \mid Y_{k}^{\tau}\right) \cdot H\left(X \mid Y_{k}^{\tau}\right)}}
$$

A partir desses dois estimadores (Equações 3.16 e 3.17) normalizados e com erros de desvios compensados, obtém-se uma relação algébrica que representa a quantidade de informação reduzida ao se inserir a $T E$ sobre a $D M I$ como medida de associação. Escreve-se esta relação como a diferença normalizada, $\triangle \widehat{T E}$, a partir da $D M I$ e da $T E$ (Besson et al., 2010).

$$
\Delta \widehat{T E}(X \rightarrow Y)=\frac{\widehat{I}_{B}\left(X ; Y^{\tau}\right)-\widehat{T E}_{B}(X \rightarrow Y)}{\widehat{I}_{B}\left(X ; Y^{\tau}\right)}
$$

A Equação 3.18 mede o nível da transferência de informação, o qual possui valores entre $0 \mathrm{e}$ 1. Essa medida melhora a interpretação de resultados entre diferentes amostras e experimentos entre si. $\mathrm{O}$ valor máximo do nível de transferência tende a 1 , para $\widehat{T E_{B}}(X \rightarrow Y)=0$. Isso 
ocorre, quando a $T E$ possui valores próximos à linha de base, significando que a direção da informação é menor neste sentido. Quando $\Delta \widehat{T E}(X \rightarrow Y)$ tende a 0 , tem-se que a direção de transferência de informação é maior e possui uma direção da transferência de informação predominante em $X \rightarrow Y$ (Jin et al., 2010). 


\section{Capítulo 4}

\section{Materiais e métodos}

Este capítulo apresenta os principais métodos e algoritmos para a obtenção dos resultados de medidas de associação e transferência de informação. Para se ter um visão geral do sistema neural proprioceptor do inseto, é mostrado na Figura 4.1 um modelo dos tipos de sinais e das conexões neurais, com base na descrição biológica em Burrows (1996).

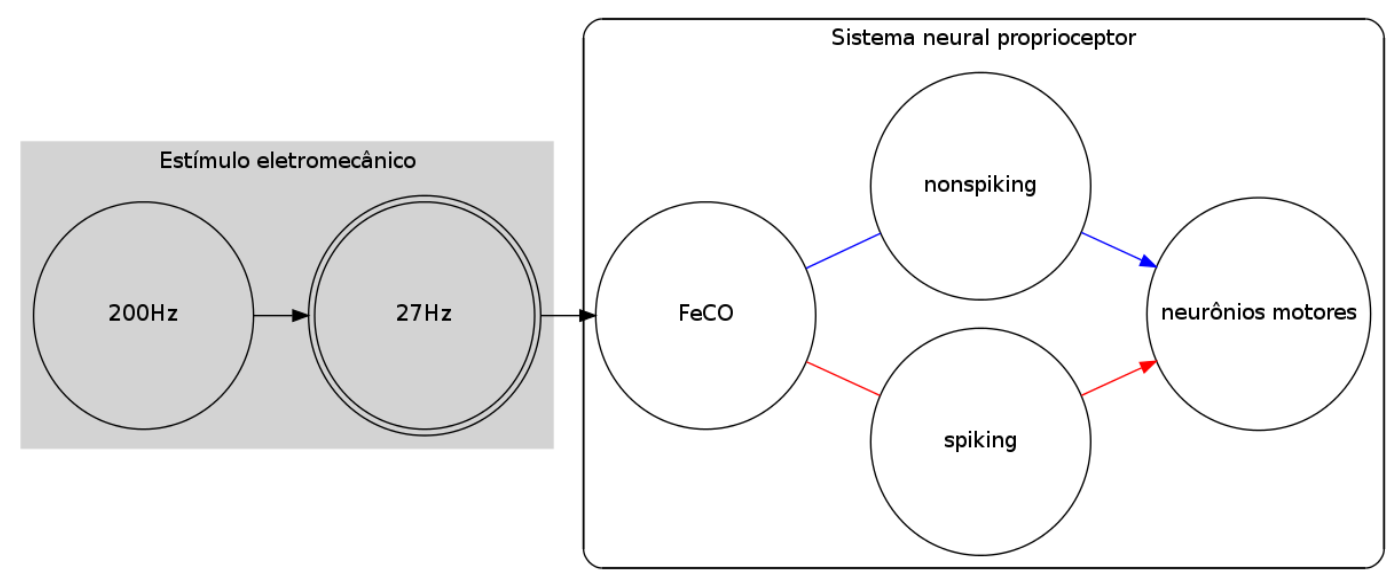

Figura 4.1: Modelo geral para o método de estímulo e coleta intracelular de sinais do sistema neural do inseto. Visão geral do sistema neural proprioceptor do inseto, a partir do estímulo elétrico de $200 \mathrm{~Hz}$, o mecânico de $27 \mathrm{~Hz}$ e as regiões neurais do gafanhoto.

O esquemático de coleta de dados é descrito na Figura 4.1 e tem seu modelo biológico apresentado, anteriormente, na Figura 2.8 da Subseção 2.3.1. Esses sinais neurais não foram todos coletados simultaneamente. E, devido essa limitação da coleta de dados, tem-se disponíveis somente pares de sinais coletados ao mesmo tempo, entre o estímulo eletromecânico (sinais de $200 \mathrm{~Hz}$ ou $27 \mathrm{~Hz}$ ) e algum ponto do sistema neural proprioceptor. Desta forma, a principal abordagem metodológica é a criação de um procedimento de análises computacionais 
que busca estimar as inferências sobre cada parte do sistema neuromotor. Pois um dos objetivos deste trabalho é comparar e analisar de forma conjunta as diferentes características de cada região neural.

Inicialmente, são analisados os sinais neurais da região sensorial que envolve o estímulo eletromeânico e o $\mathrm{FeCO}$. Nesta primera abordagem, é utilizada a análise do STA que busca estabelecer uma medida de associação de primeira ordem, entre o estímulo mecânico do apódema e os neurônios sensores do $\mathrm{FeCO}$. Para isso, determinam-se as relações entre as curvas de estímulo e a geração de spikes. Assim, é proposto um algoritmo específico para a análise do STA. Para os neurônios sensores, também é realizada a abordagem de transferência de informação com a $D M I$ e a $T E$, a fim de se quantificar a informação transmitida ao neurônios sensores.

Na segunda etapa, são analisados os sinais dos interneurônios que recebem os sinais sensores do $\mathrm{FeCO}$. No entanto, no banco de dados não se tem disponível uma coleta simultânea entre estes dois tipos de neurônios. Foram coletados sinais entre o estímulo e os sinais neurais dos interneurônios spiking e nonspiking, separadamente.

Por último, busca-se mapear um modelo estrutural único do circuito neuromotor do gafanhoto. Para isso, são propostas medidas de normalização para se mapear, dentro de um mesmo critério, os diferentes padrões de caminho que ocorrem no sistema proprioceptor do inseto. A esses padrões estão associadas as ações motoras de inibição ou excitação do membro do inseto. Desta forma, por saber que as conexões neurais têm componentes lineares, foi utilizada a correlação cruzada para definir a relação entre as camadas neurais e as ações motoras no membro do gafanhoto.

Nas seções seguintes são apresentados os modelos matemáticos do STA, os algoritmos para a obtenção das $p d f$ 's. São, também, mostrados os pseudocódigos para o atraso da informação mútua $(D M I)$, da transferência de entropia (TE) e a geração dos Surrogate Data para a comparação e validação de resultados. São descritos o modelo dos dados simulados e os principais procedimentos para a coleta dos dados reais do sistema neuromotor dos gafanhotos. 


\subsection{Algoritmos}

A partir das estruturas e algoritmos em pseudocódigos apresentados, foram desenvolvidos códigos de programação utilizando a linguagem Python ${ }^{1}$ em ambiente Linux da distribuição do Ubuntu, versão 13.04. Todos os algoritmos são implementados em programação paralela e desenvolvidos tanto para a aplicação em clusters de computadores quanto para computadores pessoais com múltiplos processadores. Neste trabalho foi utilizado, majoritariamente, um computador desktop com processador quad-core (4 núcleos de processadores) de 2,6GHz e até 8 threads para a execução das tarefas distribuídas pelos algoritmos paralelizados. Incluindo também alguns testes no GridUNESP $P^{2}$

\subsubsection{Procedimentos para obtenção da curva média de excitação dos sinais spikes - STA}

Baseado nos conceitos apresentados no Capítulo 3, utilizou-se a Equação 3.1 em função de um tempo $\tau$ para obter-se a curva média do estímulo que gera a depolarização celular e, consequentemente, o spike. A Equação 4.1 apresenta o modelo matemático para o algoritmo do STA:

$$
\hat{A}(\tau)=\frac{1}{N} \sum_{n=1}^{N} s\left(t_{n}-\tau\right)
$$

onde $\hat{A}(\tau)$ é a curva dos valores médios do estímulo e $s$ é uma função da curva de estímulos relacionadas aos spikes, sendo $\left(t_{n}\right)$ os tempos anteriores à geração dos spikes e $\tau$ a variável da função do STA.

A Figura 4.2 ilustra os procedimentos em um diagrama esquemático para a implementação de um algoritmo que gere as curvas médias do estímulo gaussiano para a depolarização do neurônio e geração do disparo de um spike.

De acordo com o esquemático da Figura 4.2 a ocorrência de um spike, o estímulo de uma janela de tempo antecedendo o spike é registrada. Ao se definir o tamanho da janela, limita-se o valor máximo de $\tau$, em que a resposta passa a ser o resultado de uma janela de tempo de algumas centenas de milissegundos anteriores ao spike Dayan e Abbott (2001). O valor para

\footnotetext{
${ }^{1}$ Disponível em: http://www.python.org/

${ }^{2}$ Centro de Computação Científica (NCC/GridUNESP) da Universidade Estadual Paulista (UNESP) que é apoiada em parte pela FINEP e FAPESP.
} 
$\hat{A}[S T A]$
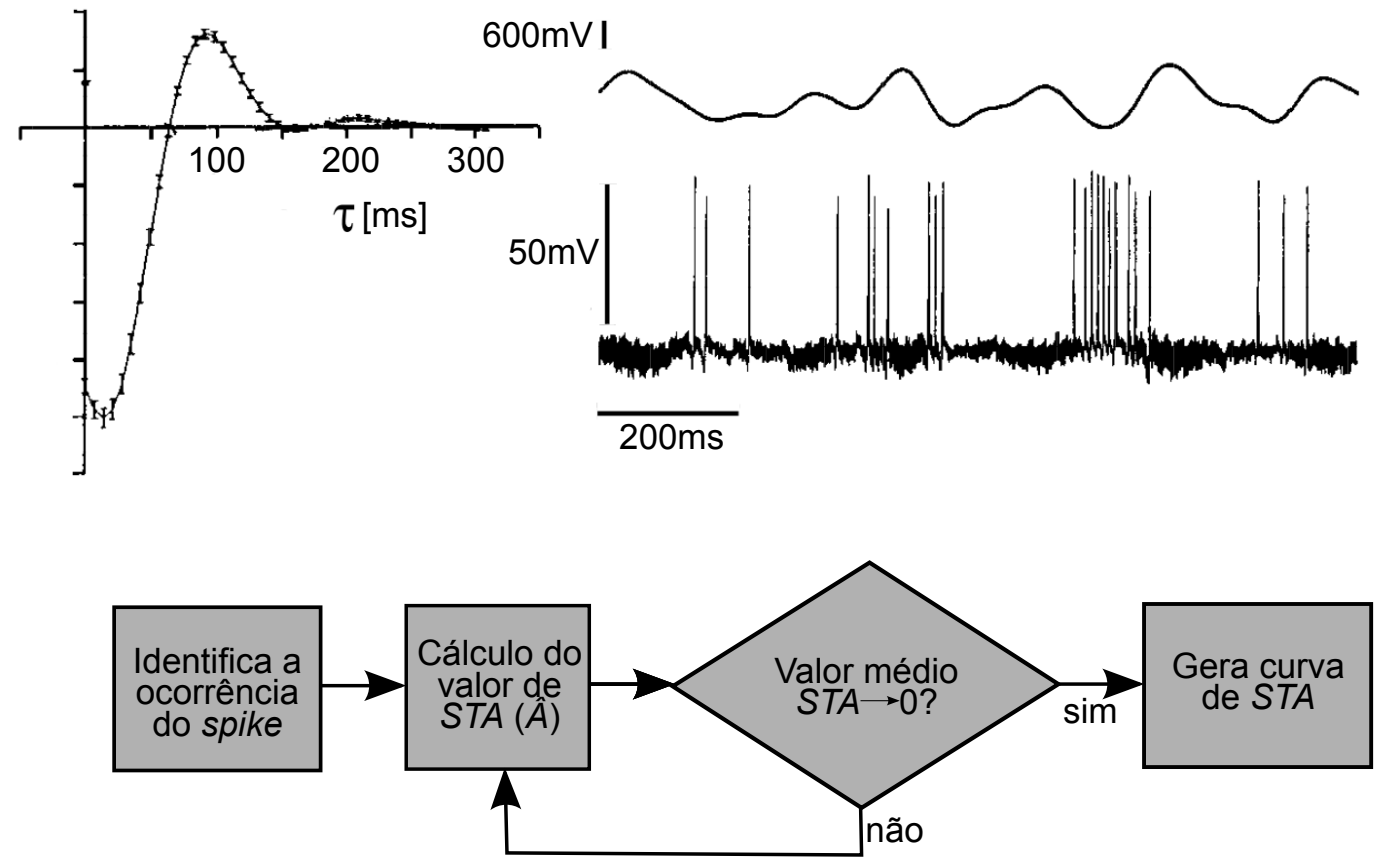

Figura 4.2: Modelo para obtenção do spike-triggered average. Descrição em diagrama de blocos para obtenção das curvas médias do estímulo gaussiano para a depolarização do neurônio e geração do disparo de um spike. [Adaptada de Dayan e Abbott (2001)]

um $\tau \ll t_{\text {spike, }}$ a correlação entre o estímulo e a resposta tende a um valor médio igual a zero. E, caso não exista correlação entre os sinais, espera-se que $\hat{A}(\tau)$ seja, também, igual a zero. Na prática, o estímulo é apenas registrado ao longo de um período de tempo finito, como visualizado na Figura 4.2. Os estímulos gravados para todos os spike são somados sucessivamente, para um valor predeterminado de $\tau$, ao longo de vários ensaios.

\subsubsection{Estimativa das funções densidade de probabilidades}

As ferramentas para a obtenção da transferência de informação dependem diretamente das funções densidade de probabilidades conjuntas. A partir da análise do STA, é possível determinar algumas probabilidades condicionadas à transmissão do sinal neural (spike) e o estímulo, utilizando as $p d f$ 's. A $D M I$ e a $T E$ utilizam diretamente as informações, através das estimativas de $p d f$ 's conjuntas de segunda e terceira ordem, respectivamente. Essas $p d f$ 's são obtidas diretamente dos dados analisados, sem utilizar parâmetros de aproximação. Em outras palavras, significa que não são utilizadas funções conhecidas para representar as distribuições de probabilidades, como as funções gaussianas, de Poisson e outras.

Matematicamente, para inferir as dependências condicionais é aplicada a regra de Bayes 
para os sinais $x$ e $y$, conforme a Equação 4.2:

$$
p(y \mid x)=\frac{p(x \mid y) \cdot p(y)}{p(x)}
$$

onde $p(y \mid x)$ é a $p d f$ a posteriori para um modelo de previsão estatística, $p(x \mid y)$ é a $p d f$ de verossimilhança sobre a informação estatística do sistema, $p(y)$ é a informação a priori e a $p(x)$ é a evidência estatística com efeito de normalização estatística do sistema.

Um exemplo para a estimativa da $p d f$ é mostrado na Figura 4.3. Ele ilustra a obtenção de uma $p d f$ de segunda ordem em que é utilizada a MI. Na Figura 4.3(a) é mostrada, a partir das $p d f$ 's marginais $p(x)$ e $p(y)$, a concentração de pontos em comum na obtenção da densidade conjunta. Utilizando-se a $M I$, obtêm-se os níveis de associação conjunta dos sinais.

Na Figura 4.3(b) são mostrados trechos selecionados das séries temporais. A escolha destes trechos é feita de forma arbitrária, tendo apenas como parâmetro a obtenção de um trecho estacionário, sem variação significativa do valor médio. A Figura 4.3(c) mostra a $p d f$ de segunda ordem com concentração dos pontos em torno de um valor médio. A informação compartilhada pelas séries temporais, utilizada para o cálculo da $M I$, é dada pela $p d f$ de $X \cap Y$.

Todas as $p d f$ 's são discretizadas em barras (bins), sendo que cada uma representa um intervalo de distribuição da ocorrência de valores das séries temporais. A discretização pode ser utilizada nas $p d f$ 's não paramétricas. A definição da quantidade de barras, para a discretização da distribuição de probabilidades tem influência direta sobre a estimativa dessas funções. Neste trabalho, foram utilizadas barras de tamanho fixo para as $p d f$ 's. Existem na literatura algumas técnicas para otimização do número de bins de acordo com o modelo de dados - uma técnica de otimização é apresentada em Shimazaki e Shinomoto (2007).

\subsubsection{Estimativa da informação mútua atrasada e da transferência de entropia}

Os algoritmos são apresentados em pseudocódigos e foram implementados todos em linguagem de programação Python. As funções dos códigos-fonte preveem a utilização de programação paralela, com o objetivo de melhorar o tempo de processamento das informações e dados biológicos.

Utiliza-se o método de estimativa de $p d f$, apresentado na subseção anterior, para cada algoritmo de transferência de informação: a $D M I$ e a $T E$. 


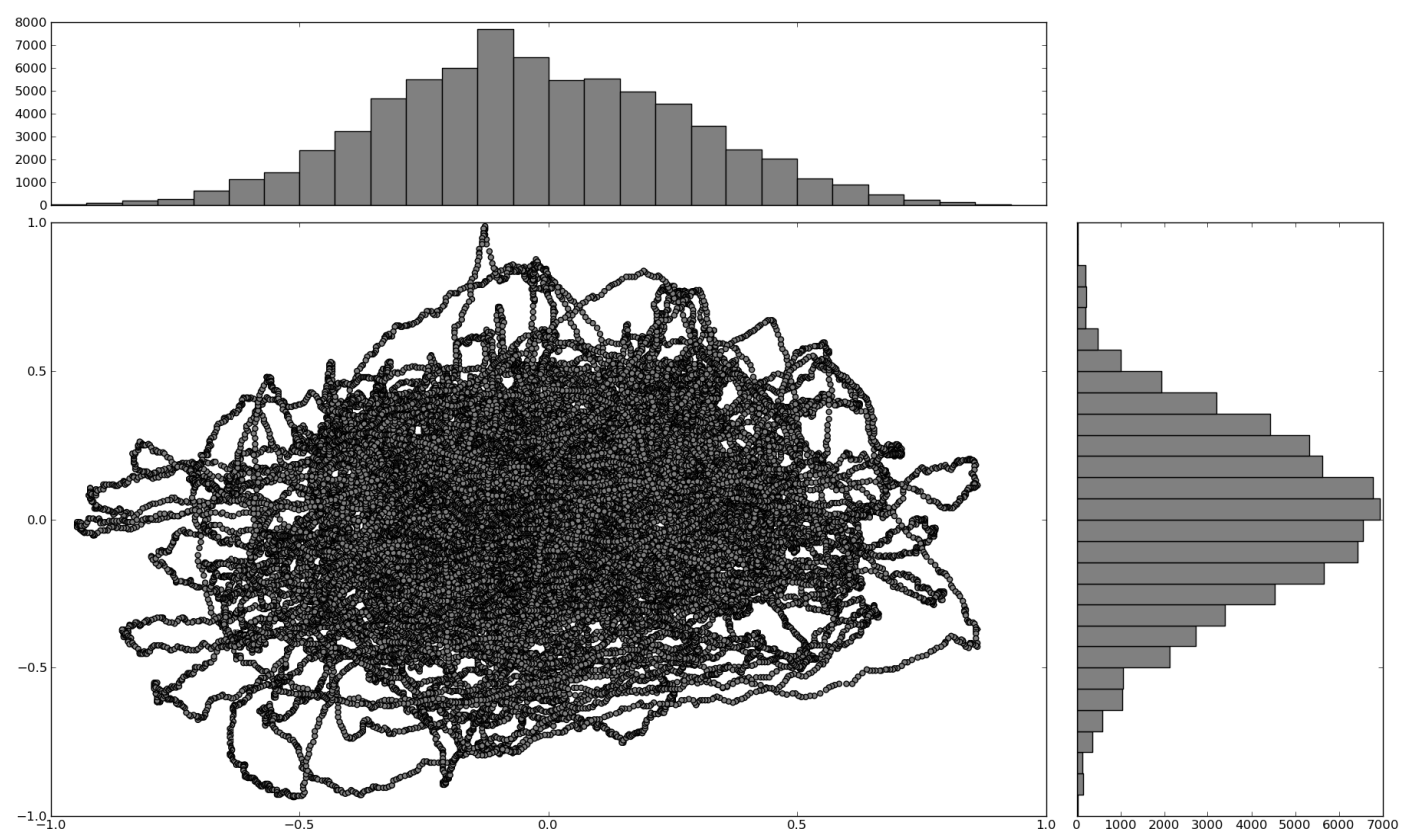

(a)
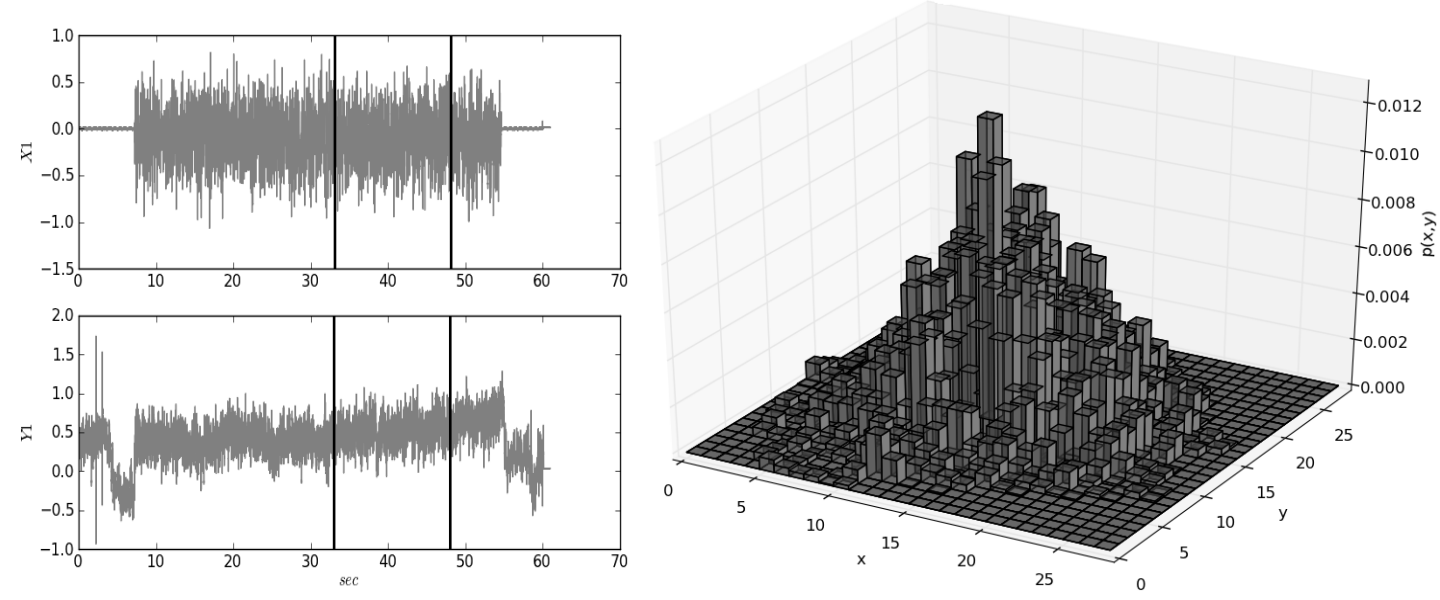

(b)

(c)

Figura 4.3: Densidades de probabilidades para a informação mútua. (a) Distribuição conjunta dos sinais das séries temporais $X$ e $Y$; no topo horizontal está a distribuição do sinal $X$ e na vertical está a distribuição do sinal $Y$. (b) Seleção (linha vertical) dos trechos estacionários das séries temporais. (c) $p d f$ conjunta de $X$ e $Y$ para cálculo da $M I$. Para a discretização das $p d f$ 's são utilizadas 28 barras (bins $=28$ ). 
O Algoritmo 1 calcula a DMI normalizada para um deslocamento dado pela variável lag, a qual representa o valor do atraso $\tau$ da Equação 3.8. A normalização é feita através do cálculo da entropia total do sistema. Nos algoritmos propostos, são aplicados os procedimentos de normalização e compensação dos níveis de flutuação da linha de base, conforme apresentado no do Capítulo 3, tanto para a DMI quanto para $T E$.

Data: Compressed plain data from input/output signals

Result: Normalized Delayed Mutual Information

def: Probabilities $(\mathrm{x}, \mathrm{y})$

return $\mathrm{p}_{x}, \mathrm{p}_{y}, \mathrm{p}_{x y}$

def: Entropies $\left(\mathrm{p}_{x}, \mathrm{p}_{y}\right)$ return $\mathrm{Hx}$, Hy

def: Mutual Information $\left(\mathrm{p}_{x}, \mathrm{p}_{y}, \mathrm{p}_{x y}\right)$ return MI

def: Delayed Mutual Information (x, y, lag) $y^{\prime} \leftarrow y$ delayed by lag px, py, pxy $=$ Probabilities $\left(\mathrm{x}, \mathrm{y}^{\prime}\right)$ $\mathrm{DMI}=$ Mutual Information $\left(\mathrm{p}_{x}, \mathrm{p}_{y}, \mathrm{p}_{x y}\right)$ return DMI

push libraries to parallel environment push function to parallel environment push variables to parallel environment

lag $\leftarrow$ min:max considerer time scatter $\{\mathrm{lag}\}$ to parallel environment $\mathrm{DMI}=$ gather Delay Mutual Information $(\mathrm{x}, \mathrm{y}, \mathrm{lag})$

$\mathrm{Hx}, \mathrm{Hy}=$ gather Entropies $\left(\mathrm{p}_{x}, \mathrm{p}_{y}\right)$ NDMI = gather Delay Mutual Information $(\mathrm{x}, \mathrm{y}, \mathrm{lag}) / \sqrt{\mathrm{Hx} . \mathrm{Hy}}$

Algoritmo 1: Descrição do algoritmo para a execução da DMI. Os comandos def/return são funções de declaração e recebimento dos valores das variáveis em processamento, e a instrução push carrega as bibliotecas, funções e variáveis nos processadores dos cluster. A instrução scatter distribui a informação através dos processos, e a instrução gather recebe a informação devolvida pelos processos.

A $T E$ é obtida de forma similar à $D M I$, com a diferença sobre a dimensionalidade das $p d f$ 's que dependem de $x_{n}, y_{n}$ e $y_{n+1}$. A Figura 4.4 mostra a seleção dos trechos das variáveis para a obtenção da transferência de entropia.

O Algoritmo 2 obtém a TE normalizada, utilizando-se a $M I$, que neste caso representa a entropia total do compartilhamento da informação entre $X$ e $Y$. O processamento desse algoritmo requer maior custo computacional por utilizar um $p d f$ de terceira ordem.

A paralelização dos algoritmos foi implementada para os diferentes tempos de atraso no 
Data: Compressed plain data from inputloutput signals

Result: Normalized Delayed Transfer Entropy

def: Probabilities $\left(\mathrm{x}_{n}, \mathrm{y}_{n}, \mathrm{y}_{n+1}\right)$

$$
\text { return } \mathrm{p}_{y_{n}}, \mathrm{p}_{x_{n}, y_{n}}, \mathrm{p}_{y_{n}, y_{n+1}}, \mathrm{p}_{x_{n}, y_{n}, y_{n+1}}
$$

def: Mutual Information $\left(\mathrm{p}_{x_{n}}, \mathrm{p}_{y_{n+1}}, \mathrm{p}_{x_{n}, y_{n+1}}\right)$

$$
\text { return } \mathrm{MI}
$$

def: Transfer Entropy $\left(\mathrm{p}_{y_{n}}, \mathrm{p}_{x_{n}, y_{n}}, \mathrm{p}_{y_{n}, y_{n+1}}, \mathrm{p}_{x_{n}, y_{n}, y_{n+1}}\right)$

\section{return $\mathrm{TE}$}

def: Delay Transfer Entropy $\left(\mathrm{x}_{n}, \mathrm{y}_{n}, \mathrm{y}_{n+1}\right.$, lag)

$y^{\prime}, y^{\prime \prime} \leftarrow \mathrm{y}_{n}, \mathrm{y}_{n+1}$ delayed by lag

$\mathrm{p}_{y_{n}}, \mathrm{p}_{x_{n}, y_{n}}, \mathrm{p}_{y_{n}, y_{n+1}}, \mathrm{p}_{x_{n}, y_{n}, y_{n+1}}=$ Probabilities $\left(\mathrm{x}, \mathrm{y}^{\prime}, \mathrm{y}^{\prime \prime}\right)$

DTE $=$ Transfer Entropy $\left(\mathrm{p}_{y_{n}}, \mathrm{p}_{x_{n}, y_{n}}, \mathrm{p}_{y_{n}, y_{n+1}}, \mathrm{p}_{x_{n}, y_{n}, y_{n+1}}\right)$

return DTE

push libraries to parallel environment

push function to parallel environment

push variables to parallel environment

lag $\leftarrow$ min:max considerer time

scatter $\{\mathrm{lag}\}$ to parallel environment

$\mathrm{DTE}=$ gather Transfer Entropy $\left(\mathrm{x}_{n}, \mathrm{y}_{n}, \mathrm{y}_{n+1}, \mathrm{lag}\right)$

$\mathrm{MI}=$ gather Mutual Information $\left(\mathrm{p}_{x_{n}}, \mathrm{p}_{y_{n+1}}, \mathrm{p}_{x_{n}, y_{n+1}}\right)$

NDTE $=$ gather Transfer Entropy $\left(\mathrm{x}_{n}, \mathrm{y}_{n}, \mathrm{y}_{n+1}\right.$, lag $) / \sqrt{\mathrm{MI}}$

Algoritmo 2: Descrição do algoritmo para a execução da TE. O algoritmo 2 é bastante similar ao desenvolvido para a $D M I$, com a principal diferença sobre a dimensionalidade das $p d f$ 's, que dependem de $x_{n}, y_{n}$ e $y_{n+1}$ 


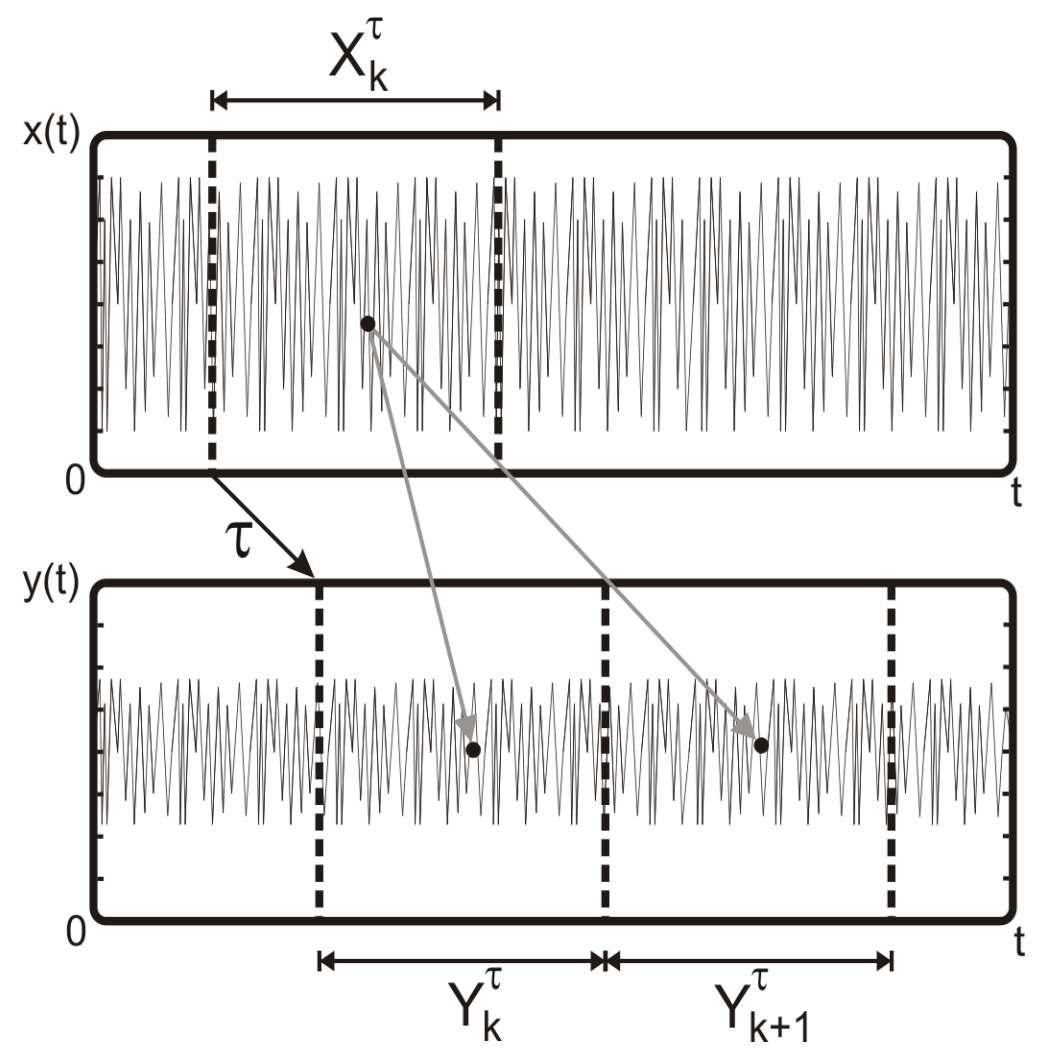

Figura 4.4: Modelo de análise das séries temporais para a transferência de entropia. São representadas as definições dos trechos de análise para a $T E$.

cálculo da transferência de informação, utilizando-se a variável lag. As funções para o cálculo da $D M I$ e da $T E$ são distribuídas entre os processos. Em seguida, os resultados dessas funções são reunidos e utilizados para a obtenção da curva de atraso da máxima coerência da informação para ambos os algoritmos.

\subsubsection{Determinação do Surrogate Data}

Para cada série temporal original, com os dados de excitação e o sinal neural do inseto, são geradas cerca de 30 séries como Surrogate Data. Essa quantitade é suficiente para um estudo comparativo e estatisticamente significativo, atendendo às recomendações, conforme descrito em (Schreiber e Schmitz, 2000).

A obtenção do Surrogate Data é baseada na IAAFT (Iterative Amplitude Adjusted Fourier Transform) (Dolan e Spano, 2001). O Algoritmo 3 utiliza o espalhamento espectral dos sinais a partir da transformada de Fourier. Inicialmente, é calculado o espectro de potência da série original para que, após as iterações, o Surrogate Data também possua o mesmo espectro. A diferença entre o Surrogate Data e a série temporal original está nas distribuições de suas 
(a)

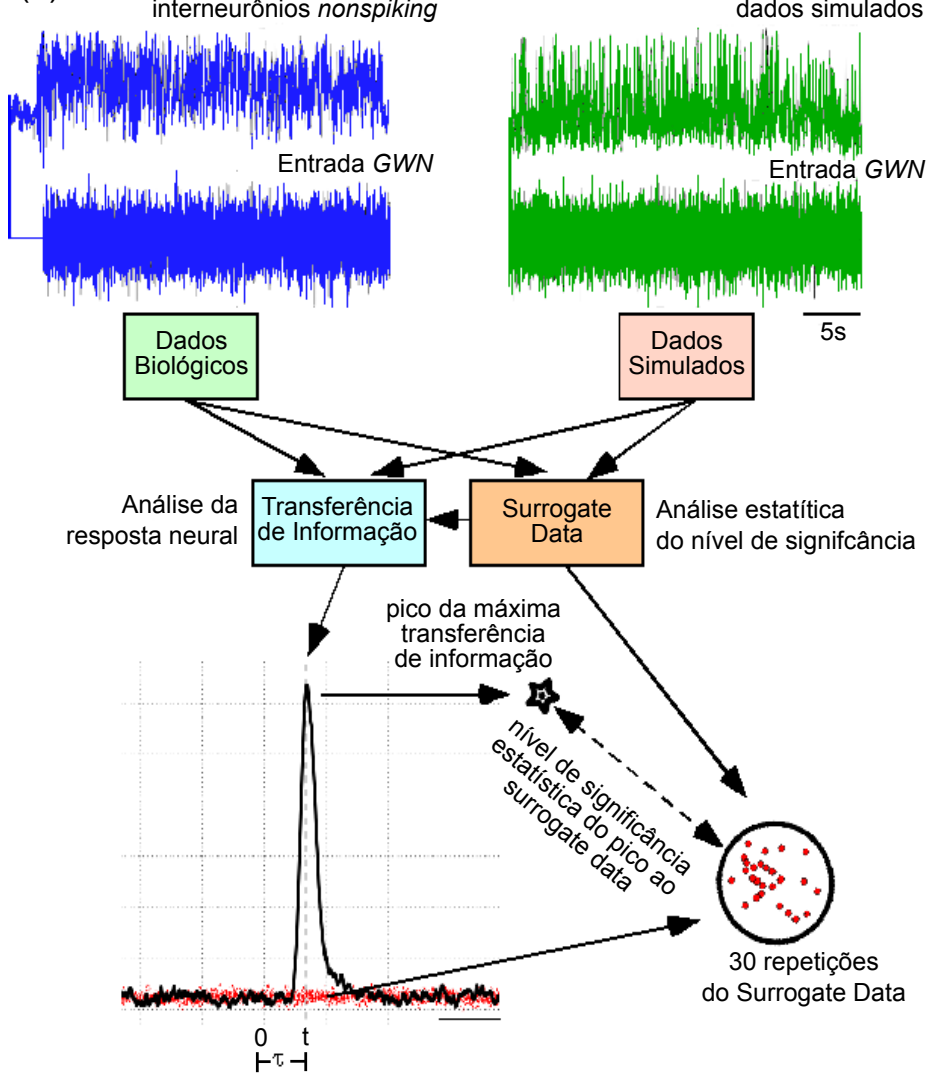

(b)
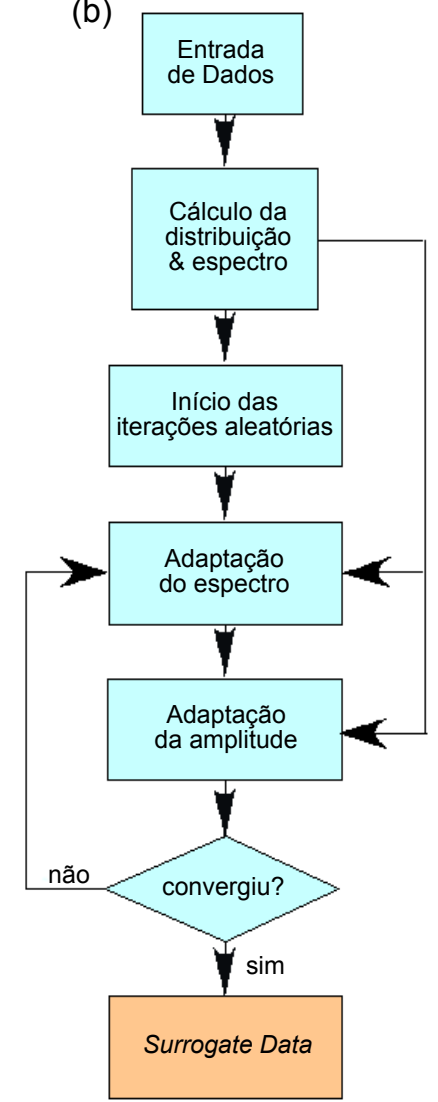

Figura 4.5: Procedimentos para geração do Surrogate Data e estimativas dos níveis de significância para comparação e validação dos resultados de máxima transferência de informação. (a) Esquemático para a estimativa da transferência de informação entre os sinais de entrada e saída, que, então, são testados com dados simulados e os dados reais biológicos. Adotaram-se níveis de significância, em 97\%, em relação aos Surrogate Data, com mesmo espectro de potência e de distribuição de amplitude dos dados reais. (b) Fluxograma para o processo iterativo de geração de Surrogate Data em cada experimento realizado. Foram gerados, de acordo com os critérios de nível de significância, um total de 30 séries temporais de Surrogate Data para cada dado analisado. 
amplitudes.

A paralelização do algoritmo é feita para a obtenção do Surrogate Data com o espectro de potência mais próximo do obtido pela série original. Para isso, cada processo recebe a função de gerar um Surrogate Data com seu respectivo erro mínimo (MinError), a partir da diferença de mínimos quadrados entre as amplitudes da série original e do Surrogate Data. Em seguida, pelo comando gather, são reunidos os valores de convergência (i), os erros mínimos (MinError $)$ e a série temporal $\left(X_{\text {sur }}\right)$ de cada processo. O algoritmo escolhe, como Surrogate Data ótimo, aquele que possuir o menor valor do MinError, indicando que houve a convergência na adaptação do melhor espectro de potência.

Data: Compressed plain data from time series signals

Result: Surrogate Data

def: Surrogate Data (x, "type")

if type $==$ "IAAFT"

Tol $=0.00001$

$\mathrm{I} \leftarrow \min$ :max considerer threads scatter $\{\mathrm{I}\}$ to parallel environment

$x^{\prime}$, tol $\leftarrow x$, tol distributed by I

Results = gather AAFT (x, Tol, I)

minerror $=\min$ (MinError)

i, MinError, $X_{\text {surro }}=$ Results ([.], minerror, [.])

return $\mathrm{X}_{\text {surro }}$

def: $A A F T(\mathrm{x}, \mathrm{Tol})$

return $\mathrm{i}$, MinError, $\mathrm{x}_{\text {surro }}$

push libraries to parallel environment

push function to parallel environment

push variables to parallel environment

$\mathrm{X}_{\text {sur }}=$ Surrogate Data $(\mathrm{x}$, "IAAFT")

Algoritmo 3: Descrição do algoritmo para a geração do Surrogate Data. O algoritmo está baseado na IAAFT (Iterative Amplitude Adjusted Fourier Transform) para a obtenção do dados com mesmo espectro de potência e diferentes distribuições.

Com base na Equação 3.14, o nível de significância foi definido para DMI e TE. No caso de o valor de $S$ ser maior do que 1,98, a partir do teste de hipótese nula, rejeitam-se os valores e considera-se que é estabelecida uma associação entre as séries temporais com 0,95 de nível de significância. 


\subsection{Descrição e modelos dos dados}

Os dados simulados para testes de validação e comparação de resultados foram gerados computacionalmente aplicando-se diferentes níveis de associação entre si. A obtenção dos dados reais, a partir dos experimentos no sistema neuromotor de gafanhos, foi realizada na Universidade de Southampton, UK, pelo Laboratório de Ciências, liderado pelo professor Philip L. Newland. Os dados reais estão disponibilizados em um banco de dados com os arquivos dos experimentos com os insetos.

\subsubsection{Dados simulados}

Para se realizar os testes e ensaios de validação dos algoritmos, foi proposto um modelo de séries temporais gerado por computador, em que são definidos o nível de associação e a função de conectividade. As duas séries temporais, $X_{t}$ e $Y_{t}$, são baseadas em um modelo proposto por Jin et al. (2010):

$$
\begin{aligned}
X_{t} & =a_{x} \cdot X_{t-1}+n_{X} \\
Y_{t} & =a_{y} \cdot Y_{t-1}+\zeta_{X Y} \cdot f\left(X_{t-1}\right)+n_{Y}
\end{aligned}
$$

O nível de associação entre as duas séries temporais é representado por $\zeta_{X Y}$, e a função de conectividade é definida por uma relação não linear, $f(x)=x^{2}$. Os valores de $\zeta_{X Y}$ são definidos no intervalo, $0,0 \leq \zeta_{X Y} \leq 1,0$, onde 1 representa a associação máxima e 0 a descorrelação entre as séries temporais. Para a análise do efeito de erro propagado devido ao tamanho das amostras, utilizaram-se diferentes tamanhos de amostras para cada uma das séries temporais em um intervalo de $0 \leq t \leq 0,8 s$ e $0 \leq t \leq 10 \mathrm{~s}$, com $F_{s}=10 \mathrm{kHz}$. As séries temporais estão sujeitas a um ruído aleatório branco, com distribuição gaussiana representada por $n_{X}$ e $n_{Y}$.

Aplicando-se o Surrogate Data para estimar as relações de máxima coerência na transferência de informação, foram utilizadas 35 séries geradas pelo algoritmo do Surrogate Data para cada uma das séries temporais. Em seguida, calcularam-se os valores máximos da $D M I$ e da $T E$, com as 10 séries de tamanhos diferentes de amostras, e as outras 10 séries com variações dos níveis de associação. Todos os valores estimados são normalizados com as entropias de cada caso, tendo como base as Equações 3.16 e 3.17. 


\subsubsection{Dados reais}

Nesta subseção, é descrita a parte experimental sobre a coleta dos sinais no gânglio metatorácico e no sistema neuromotor de gafanhotos. A partir disso, é criado um banco de dados com todos os experimentos realizados, os quais são utilizados como dados reais, para aplicação dos algoritmos e técnicas propostas neste trabalho. São descritas a organização e a forma de apresentação desses dados.

\section{Descrição do experimento com gafanhotos}

Os experimentos foram realizados no Centro de Ciências Biológicas (Centre for Biological Sciences) da Universidade de Southamptom, UK. É utilizada uma única espécie de inseto: o gafanhoto do deserto (Schistocerca gregaria). A coleta dos sinais é feita com o animal vivisseccionado e mantido sob condições em que são mantidas as suas funções neurofisiológicas regulares.

No experimento, os gafanhotos são fixados pela parte ventral-lateral superior em uma cera, semelhante a uma massa de modelar. A fixação tem por objetivo imobilizar o animal para que a movimentação de outras partes do inseto tenha mínima influência nos sinais neurais do gânglio metatorácico. Somente o membro posterior (fêmur e tíbia) é deixado livre para os movimentos que são realizados durante o experimento, além de se evitar a contração involuntária dos músculos dessa região.

Na região da junção do membro posterior do inseto, é feita uma abertura para acesso ao apódema e ao $\mathrm{FeCO}$. O apódema é exposto ao se abrir essa pequena janela na cutícula sobre a parte distal-anterior do fêmur do gafanhoto (Kondoh et al., 1995). Através do movimento forçado na região do apódema, são simulados os movimentos de flexão e extensão da tíbia. Para isso, são inseridas as pontas de um tipo de pinças finas (o fórceps), as quais estão ligadas a um equipamento que gera a excitação mecânica (Ling Altec 101). As pinças seguram o apódema do órgão cordotonal excitando-o com um movimento mecânico linear aleatório. Essas curvas de deslocamento possuem uma distribuição aleatória gaussiana.

O sinal mecânico é obtido após a filtragem de uma sequência binária pseudoaleatória (CG742, NF Circuit Design Block) com largura de banda de $200 \mathrm{~Hz}$, sendo um ruído gaussiano branco (GWN - Gaussian White Noise). O sinal de $200 \mathrm{~Hz}$ é filtrado por um filtro passabaixas, Chebyshev, de quarta ordem (SR-4BL, NF Circuit Design Block), com um tempo de 
atraso estimado em $15 \mathrm{~ms}$ e com decaimento na frequência de corte em torno de $24 \mathrm{~dB}$ / oitava (Vidal-Gadea et al., 2010). A partir da obtenção desses sinais, o fórceps recebe o sinal $G W N$ filtrado com largura de banda de $27 \mathrm{~Hz}$ e provoca o movimento de deslocamento mecânico para a movimentação do apódema. A Figura 4.6 apresenta o diagrama de blocos da geração e filtragem do sinal da excitação eletromecânica.

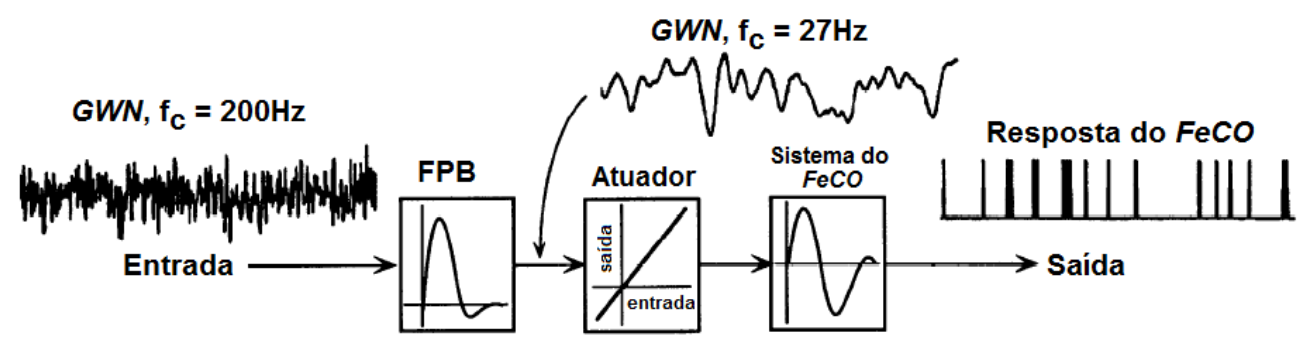

Figura 4.6: Diagrama de blocos da geração dos sinais eletromecânicos para a excitação do apódema do $\mathrm{FeCO}$. [Adaptada de Vidal-Gadea et al. (2010)]

Existe uma curva característica conhecida de transferência entre o movimento do apódema e o movimento real da junção femoro-tibial. Um movimento de $500 \mu m$ do apódema corresponde a um deslocamento angular de $40^{\circ}$ da junção. A relação entre o movimento e deslocamento angular é linear dentro do intervalo definido em: $20^{\circ} \leq \theta \leq 130^{\circ}$ (Burrows, 1987). Dessa forma, o apódema provoca um sinal neural de excitação nos neurônios sensores na região do $\mathrm{FeCO}$, em que o relaxamento do apódema é equivalente à extensão da junção, e a extensão do apódema equivalente à flexão da junção (Burrows et al., 1988). A Figura 4.7 mostra o esquema experimental para o acesso ao apódema do $\mathrm{FeCO}$ para a excitação e coleta dos sinais no sistema neuromotor do gafanhoto. Todos estes procedimentos encontram-se, com mais detalhes, em: Kondoh et al. (1995), Newland e Kondoh (1997a) e Newland e Kondoh (1997b).

A partir desse experimento, são coletados os dados através de eletrodos fixados em diferentes partes do sistema neuromotor do inseto. São obtidos os sinais dos neurônios sensores $(\mathrm{FeCO})$ localizados no fêmur e dos demais neurônios no gânglio metatorácico. No gânglio, estão localizados os interneurônios locais (spiking e nonspiking) e os neurônios motores que inervam os músculos do membro posterior.

Os sinais são coletados a partir de 4 canais, simultaneamente, para os sinais de $200 \mathrm{~Hz}$, $27 \mathrm{~Hz}$, alguma região aleatória do circuito neural e para o neurônio em análise. Os sinais digitalizados são coletados com frequência de amostragem, $F_{s}$, de $10 k H z$ ou $24 k H z$ (Kondoh et al., 1995), (Newland e Kondoh, 1997a) e (Newland e Kondoh, 1997b). Dessa forma, cada experimento tem de ser verificado e analisado de acordo com a frequência de amostragem com 


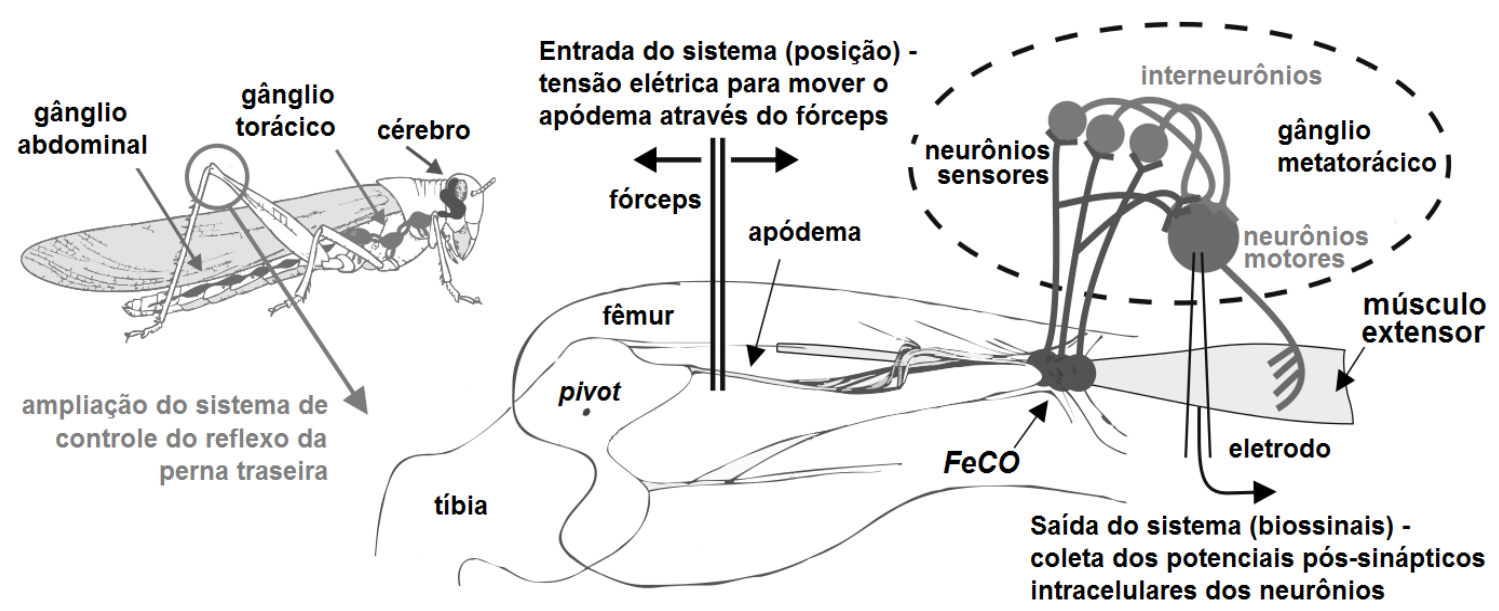

Figura 4.7: Esquema experimental para excitação dos neurônios motores através do deslocamento mecânico do apódema femorotibial. [Adaptada de Dewhirst et al. (2009)]

que foi coletada.

Para a análise e obtenção dos resultados, são utilizadas três das quatro séries temporais obtidas de cada experimento. Na Figura 4.8 são mostrados dois exemplos do conjunto de sinais utilizados nas análises de transferência de informação. Na Figura 4.8(a) é apresentado um experimento para os neurônios com sinal spiking e na Figura 4.8(b) para um sinal nonspiking. As duas figuras apresentam as excitações, em $200 \mathrm{~Hz}$ e $27 \mathrm{~Hz}$, que geram os seus respectivos sinais neurais.

Todos os dados reais estão organizados em um banco de dados com todos os experimentos realizados.

\section{Banco de dados dos experimentos}

Ao se realizar cada experimento, todos os dados são gravados em arquivos eletrônicos e identificados por tags alfanuméricas (exemplo: $I D-923$ ). O arquivo de um conjunto de sinais coletados é mostrado na Figura 4.9(a). A gravação de cada arquivo conta com os sinais obtidos durante todo o experimento, desde o momento da inserção dos eletrodos e o início da geração dos sinais de excitação, até a finalização de cada experimento. 

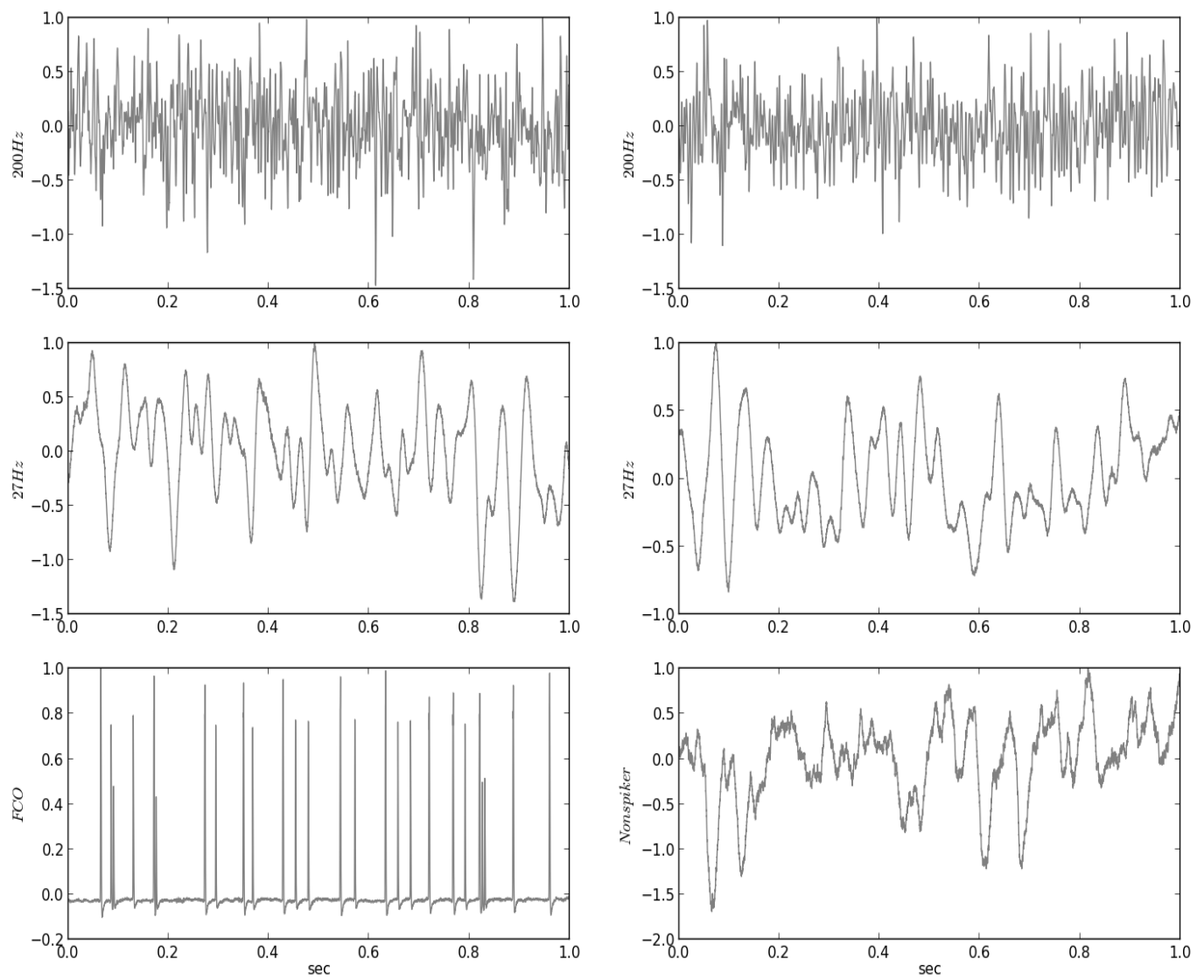

(a)

(b)

Figura 4.8: Amostra de 1 segundo da coleta de dados do experimento com gafanhotos. São mostrados os sinais de $200 \mathrm{~Hz}, 27 \mathrm{~Hz}$ e os exemplos de um sinal do tipo (a) spiking e (b) nonspiking. Nos dois casos os sinais são coletados com frequência de amostragem $F_{s}=$ $24 k H z$, no entanto, alguns sinais também são coletados com, $F_{s}=10 k H z$. 

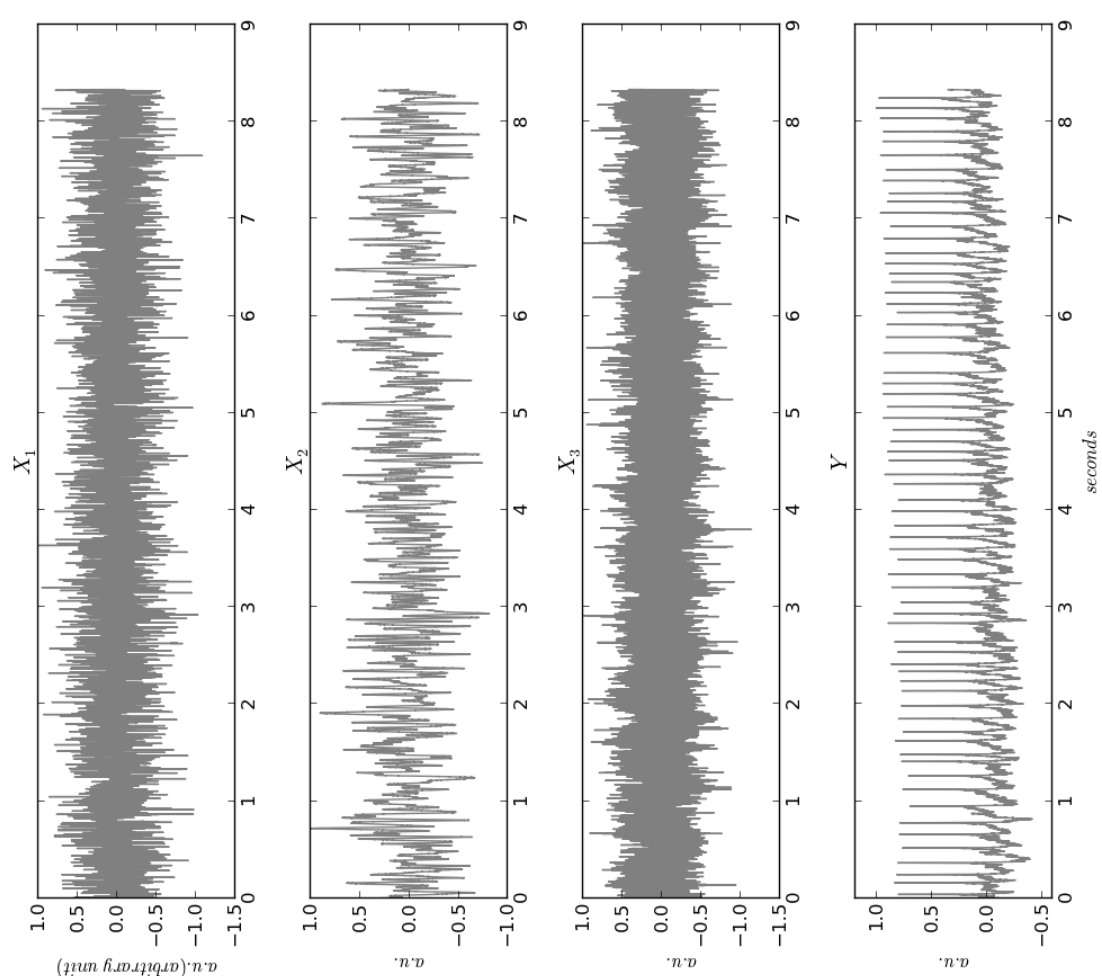

怘焉

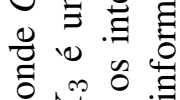
के ह 总点跑

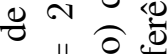
\& $\|$ 导范 ปี⿻ ప 요요 है : อ

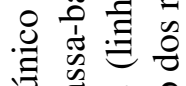

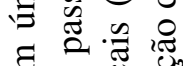
छ ฮ औ 0 जै 0 흘 总范 छี

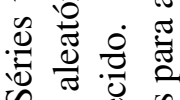
थ

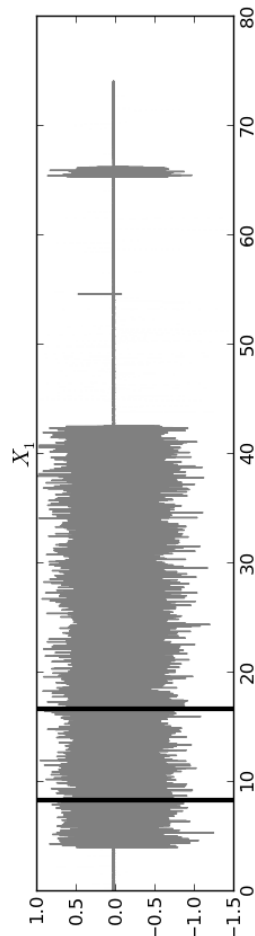

(zun f..tv.lpqu..s $) \cdot n \cdot v$
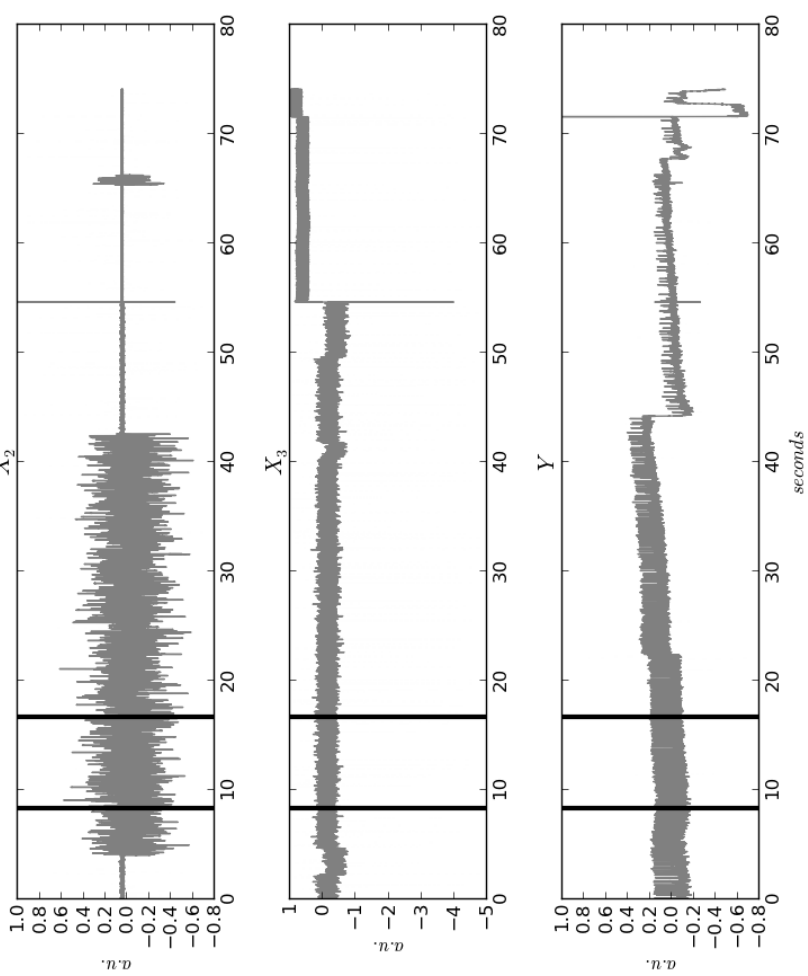

$\dot{s}$ 용요 च $\Xi$ न 记 兰 。 \& 论 สิ

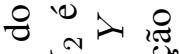

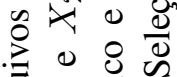
当 可部苞

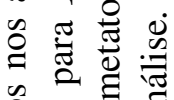
语 N 䨔 ๘ั .

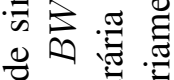
융 흏 5 을

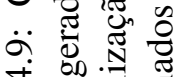
ㄱำ 휴 클 江 0 \% 
Na Figura 4.9(b) é mostrado o intervalo selecionado para as análises de transferência de informação. Todos os trechos selecionados são estacionários no sentido amplo (WSS - Wide Stationarity Sense), para isso foram utilizados os algoritmos de testes de estacionariedades desenvolvidos e apresentados em Nicácio et al. (2012). Essas amostras são selecionadas de forma arbitrária a partir de trechos que estão sujeitos à estacionariedade conjunta entre os quatro canais. Por esse motivo, elas possuem tamanhos diferentes.

Além dos testes clássicos de estacionariedade, foi utilizada uma análise baseada na ocupação da capacidade do canal (Goldsmith e Varaiya, 1996). Pois, ao se encontrar as informações mútuas atrasadas, determina-se o intervalo temporal em que as séries temporais se mantêm associadas. Por esse motivo, garante-se um critério mais específico de estacionariedade quando se utilizam as ferramentas de teoria de informação.

Na Figura 4.10 é mostrado o trecho no tempo em que ocorre a transferência de informação, obtida pela interpretação da capacidade do canal. Para todos os experimentos os testes de capacidade do canal foram realizados, a fim de se garantir uma região estacionária do tipo WSS e com ocorrência de transferência de informação.

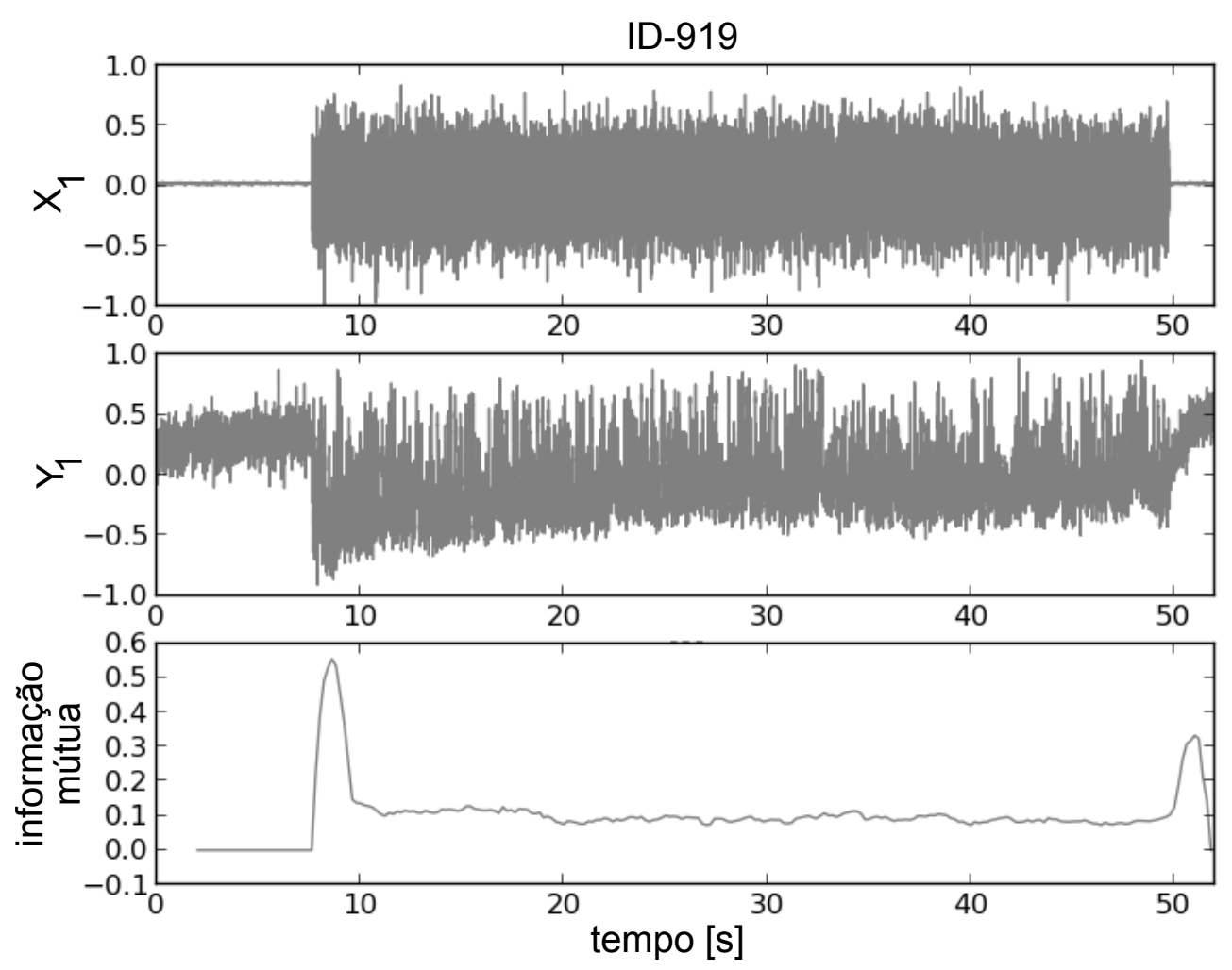

Figura 4.10: Determinação dos trechos estacionários pela capacidade do canal (em [bits]). Seleção dos trechos de séries temporais biológicas, utilizando os critérios baseados na estacionariedade WSS e para a obtenção de trechos com ocorrência da transferência de informação. 
É importante observar os transitórios de capacidade do canal, conforme mostrado na Figura 4.11 pela curva com pico de capacidade do canal alterada. Isso ocorre, principalmente devido às condições de não estacionariedade na transição dos ruídos de fundo para o início do estímulo através do sinal em $X_{1}$. Demonstrando, assim o início da atividade neural estimulada no neurônio em estudo.

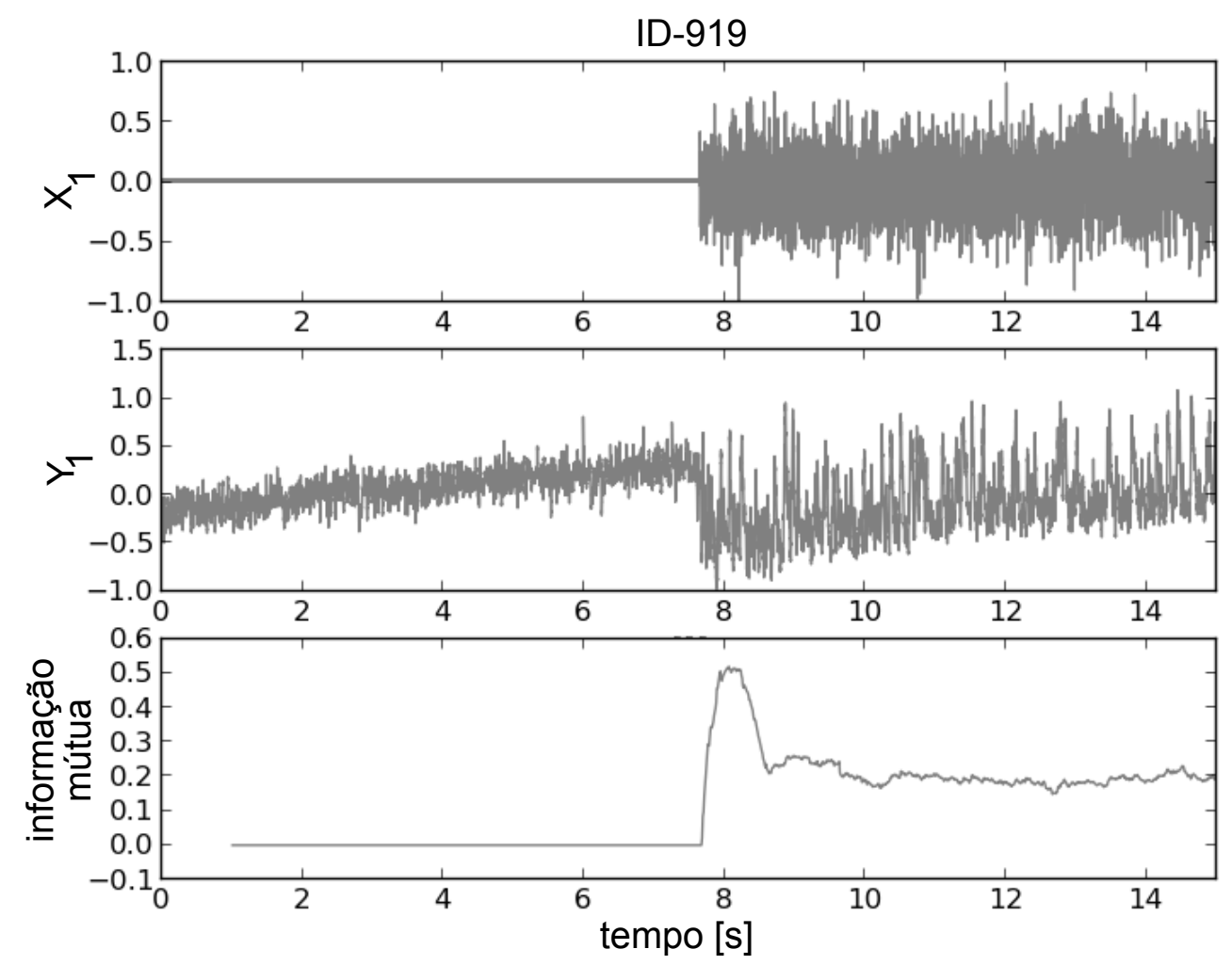

Figura 4.11: Pico de transição da capacidade do canal (em [bits]) no início do estímulo neural. Visualiza-se na transição entre o ruído de fundo (sem a presença de estímulo gaussiano) e o início do estímulo através do sinal em $X_{1}$.

O banco de dados é formado por cerca de 325 experimentos. Cada algoritmo desenvolvido neste trabalho faz a leitura de um arquivo de dados em que são escolhidos os experimentos a serem analisados. Os arquivos são divididos por lotes de acordo com o tipo do neurônio: neurônios sensores do $\mathrm{FeCO}$, interneurônios spiking e interneurônios nonspiking (disponíveis no Anexo A). Os algoritmos fazem as análises aos pares, pois são utilizados os sinais de excitação de $27 \mathrm{~Hz}$ e um sinal neural. Para cada um dos três lotes, são definidos os ID's a serem analisados, os canais escolhidos (excitação e o neurônio) e o intervalo da amostra.

Para auxiliar o acesso e a organização desse banco de dados, foi criado um arquivo geral de descrição dos dados (disponível no Anexo B). Nesse arquivo é identificado a ID (identificação do experimento), a frequência de excitação mecânica, a frequência de amostragem e a identi- 
ficação dos quatro canais coletados simultaneamente. O banco de dados possui experimentos realizados com excitações mecânicas de $27 \mathrm{~Hz}, 58 \mathrm{~Hz}$ e $100 \mathrm{~Hz}$; no entanto, para este trabalho, foram utilizados somente os experimentos com excitação mecânica de $27 \mathrm{~Hz}$.

\subsection{Modelo da estrutura de múltiplos padrões de caminhos da trans- ferência de informação no sistema neuromotor}

Nesta seção, é apresentado o modelo da estrutura básica do circuito neural para as respostas dos reflexos locais do membro posterior do inseto, quando este é estimulado pelo $\mathrm{FeCO}$. Em cada caminho do sinal neural, espera-se que o circuito provoque um atraso na transferência de informação.

As respostas esperadas são originadas no $\mathrm{FeCO}$ (sinais de entrada) e chegam até aos interneurônios que atingem, por fim, os neurônios motores (neurônios de saída). Na Figura 4.12, são identificados pelo menos três padrões de caminhos até os neurônios motores. O primeiro tem uma conexão direta com os neurônios sensores do $\mathrm{FeCO}$ e provoca a excitação destes neurônios de saída. O segundo padrão de caminho ocorre através dos interneurônios nonspking, que excitam o neurônio motor. A excitação ocorre pela sobreposição de duas inibições seguidas (Burrows, 1987), (Burrows et al., 1988). O terceiro padrão de caminho acontece através dos interneurônios spiking, que provocam a inibição do neurônio motor.

\section{neurônios interneurônios neurônios sensores spiking nonspiking motores}

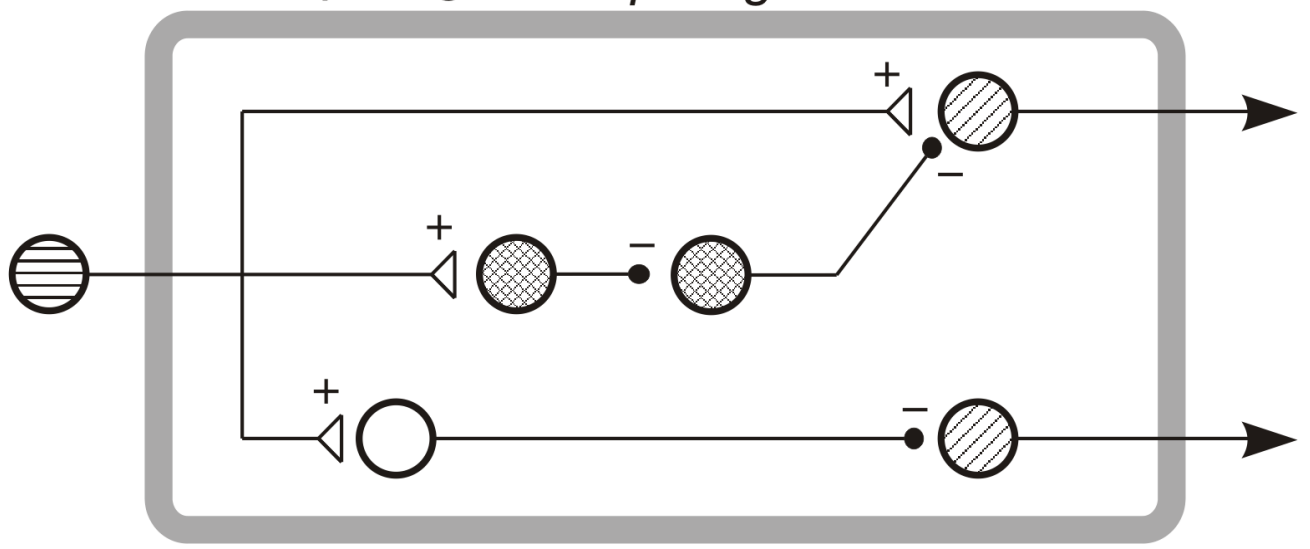

Figura 4.12: Circuito neural de resposta local entre o $\mathrm{FeCO}$ e os interneurônios. Este é o modelo esperado através do estudo fisiológico da estrutura de reflexo local de acionamento pelo FeCO. [Adaptada de Burrows (1996)] 
No entanto, em outros estudos como em Dewhirst et al. (2009) e Vidal-Gadea et al. (2010), observou-se que o modelo de múltiplos caminhos no gânglio torácico aponta para sobreposições de inibição do interneurônios spiking, conforme é melhor descrito na Figura 4.13(a). Este circuito neural mostra com mais detalhes as camadas de interneurônios responsáveis pela resposta propriceptora do inseto. Da mesma forma, como comentado anteriormente, são identificados os padrões de caminhos sinápticos a partir dos neurônios sensores do $\mathrm{FeCO}$, através dos interneurônios spiking. No entanto, a principal diferença está nas formas como os interneurônios nonspiking podem ser controlados.

Primeiro, o nonspiking pode ser excitado por sinapses diretas dos neurônios sensores, no qual existe apenas um padrão de caminho identificado. Em segundo, o interneurônio nonspiking pode inibido diretamente por meio de uma única camada dos interneurônios spiking, com a ocorrência de dois padrões de caminho do neurônio sensor até o nonspiking. Por último, pode ocorrer uma sobreposição de inibições por meio de duas camadas dos interneurônios spiking.

A análise do modelo de circuito neural é relevante para a classificação e obtenção dos múltiplos atrasos da transferência da informação, pois, ao se aplicarem as ferramentas de DMI e da $T E$, são identificados padrões característicos das camadas neurais no circuito neural do inseto.

Todos os sinais gerados pela excitação eletromecânica têm sua informação transmitida chegando até os neurônios motores através do interneurônios. No entanto, o padrão de cada caminho não pode ser definido somente com a parte experimental fisiológica. Os experimentos distinguem, apenas, a localização do neurônio, com base na sua estrutura anatômica. Assim, não é possível prever-se os múltiplos caminhos. Para isso, são propostos alguns métodos computacionais e algoritmos para a caracterização e mapeamento dos sinais percorridos no circuito neural do inseto.

Utilizou-se um algoritmo baseado no critério de informação bayesiana (BIC - Bayesian Information Criteria), a partir de misturas de gaussianas 2-D (Schwarz (1978) apud ZakSzatkowska e Bogdan (2011)) para classificação desses dados e identificação de múltiplos caminhos. O cálculo do BIC utiliza a seguinte equação:

$$
B I C=-2 \cdot \ln (\hat{L})+\eta \cdot \ln (\kappa)
$$

onde $\hat{L}$ é uma função de máxima verossimilhança relacionada a uma distribuição a priori dos 


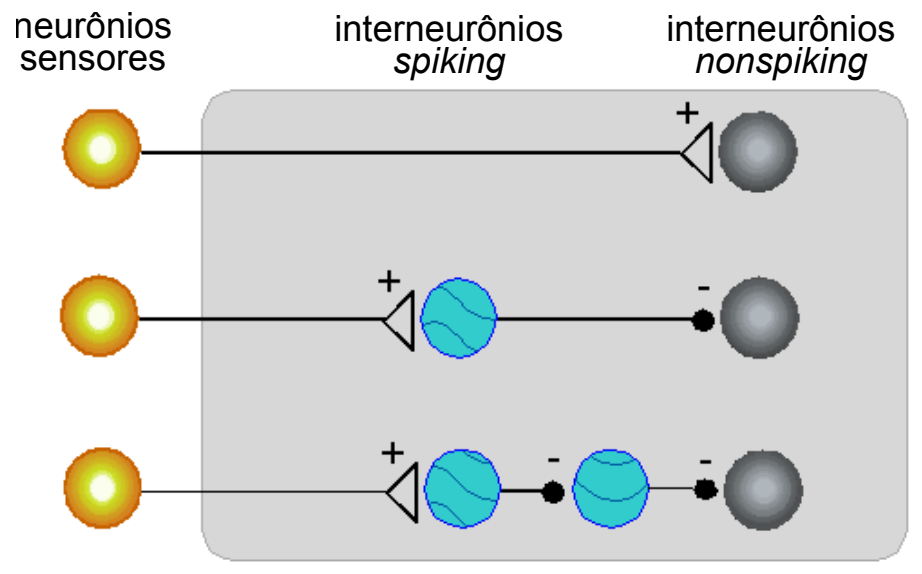

(a)
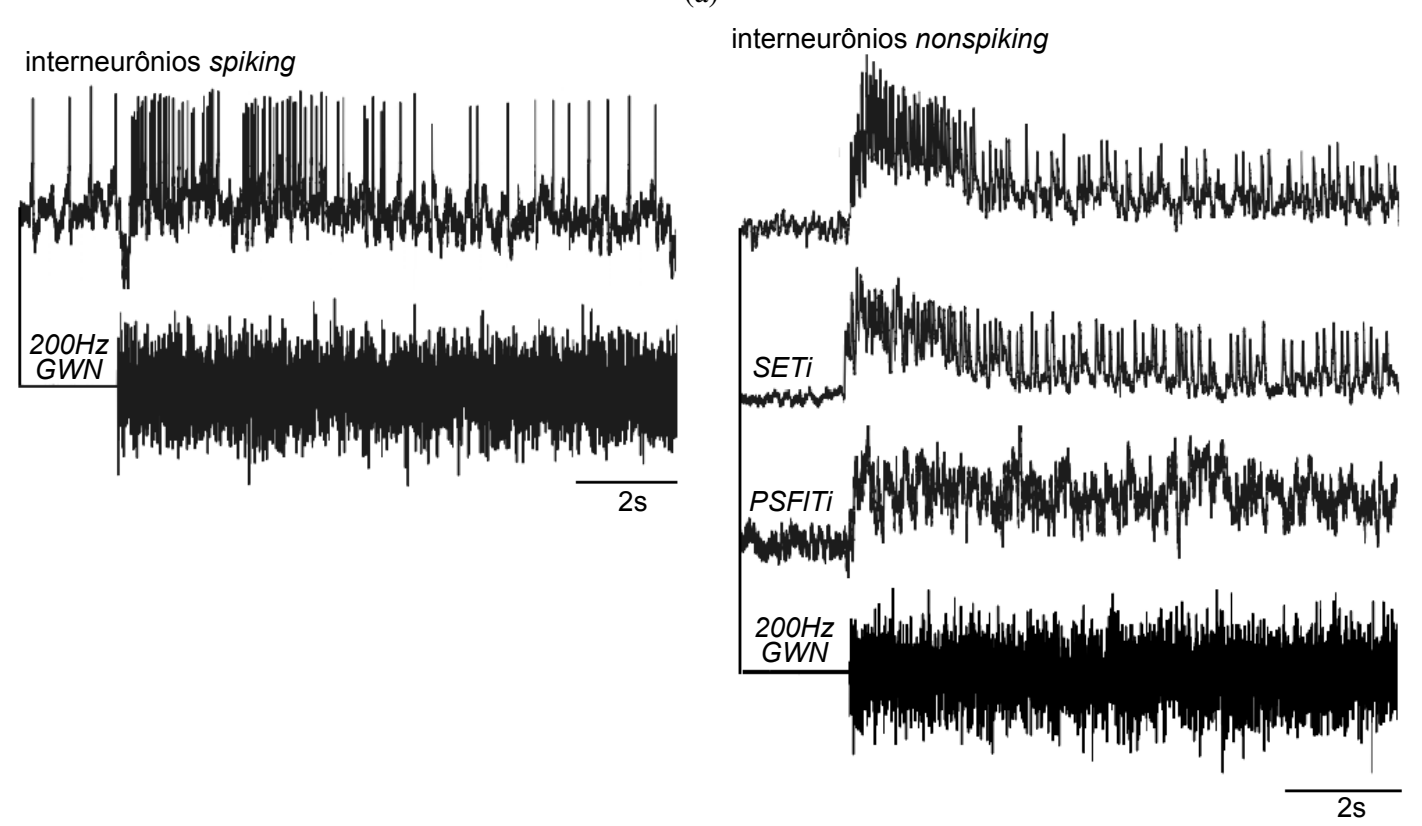

(b)

Figura 4.13: Modelos do circuito neural e dos sinais interneuronais. (a) Circuito neural que identifica os padrões de caminhos sinápticos entre os neurônios sensores do $\mathrm{FeCO}$, através dos interneurônios spiking [Adaptado de Burrows et al. (1988)]. Os interneurônios nonspiking podem ser excitados por sinapses diretas dos neurônios sensores do $\mathrm{FeCO}$ ou inibidos indiretamente por meio de uma única camada dos interneurônios spiking ou com uma sobreposição de inibições por meio de duas camadas dos interneurônios spiking. (b) Exemplos dos sinais coletados para análise das camadas e múltiplos padrões de caminho para o controle neuromotor de um gafanhoto. 
parâmetros para os dados, $\chi$. Em seguida, $\kappa$ é a quantidade de dados ou número de observações e $\eta$ o número de parâmetros a ser estimado, sendo $\chi$ os dados observados para o cálculo da variância do erro $\sigma_{e}^{2}$, dado por:

$$
\sigma_{e}^{2}=\frac{1}{\kappa} \sum_{i=1}^{k}\left(\chi_{i}-\hat{\chi}_{i}\right)^{2}
$$

onde $-2 \cdot \ln (\hat{L}) \approx \kappa \cdot \ln \left(\sigma_{e}^{2}\right)$ é interpretado como um logaritmo de máxima verossimilhança.

O valor do $B I C$ é cálculado para diferentes valores de $\eta$, a partir do conjunto de dados $\chi$. Para cada valor de $\eta$, é estimado o erro de variância, $\sigma_{e}^{2}$. O menor erro minimiza o BIC para diferentes valores de $\eta$. Dessa forma, o valor ótimo de $\eta$ parâmetros identificados ocorre para o menor valor do BIC. Com base nesse conceito, são analisados os múltiplos padrões de caminhos dos sinais neurais, a partir dos resultados de atrasos da máxima coerência da informação. 


\section{Capítulo 5}

\section{Resultados e discussões}

Neste capítulo, apresentam-se os ensaios obtidos com os dados simulados e os dados reais coletados do sistema neuromotor de gafanhotos. Primeiramente, são realizadas as análises do STA, conforme a metodologia descrita no Capítulo 4. A partir das medidas de associação dadas pelo STA, é feita uma inferência bayesiana nesses resultados. Assim, obtém-se um modelo inicial de previsão com a informação a posteriori, dada pela $p d f$, $p($ spike|stim). Ao se encontrar uma relação de probabilidades entre o estímulo e o sinal neural, o modelo se mostra promissor para se calcular a transferência de informação entre esses sinais.

Em seguida, mostram-se os resultados obtidos com as ferramentas computacionais de transferência de informação, a $D M I$ e a $T E$. Para discutir e verificar o funcionamento desses algoritmos, são utilizados os dados de simulação. Esses dados permitem que sejam verificados os efeitos do nível de associação, $\zeta_{x y}$, entre diferentes séries temporais. Por se tratar de ferramentas com base em informações estatísticas, existe a necessidade de se analisar os níveis de significância, conforme discutido na Seção 3.4. Para isso, são gerados e discutidos os resultados de Surrogate Data, também para os dados simulados. Outro aspecto importante desse tipo de análise com séries temporais biológicas é a existência de erros de desvio. Verificou-se que as informações da linha de base na transferência de informação sofrem grandes variações em seus valores absolutos. Estas variações estão relacionadas ao tamanho das amostras das séries temporais escolhidas. Dessa forma, aplicam-se as medidas de normalização e compensação apresentadas na Seção 3.5.

Após os ensaios com os dados simulados, aplicou-se a mesma metodologia para os dados reais. Esses dados incluem os sinais dos neurônios sensores, dos interneurônios do gânglio me- 
tatorácico, spiking e nonspiking do sistema proprioceptor dos membros posteriores do inseto. No entanto, para os sinais neurais, todos os efeitos sintéticos encontrados nos dados simulados têm uma interpretação neurofisilógica para o sistema neuromotor do inseto. Um dos parâmetros mais importantes encontrados pela transferência de informação são os tempos de atraso, $\tau$. Esses tempos estão diretamente relacionados aos múltiplos padrões de caminhos entre os neurônios do sistema neuromotor, conforme é discutido na Seção 4.3.

Com os tempos de atraso e juntamente com os valores de máxima transferência de informação nos diferentes tipos de neurônios, utiliza-se o classificador bayesiano $(B I C)$ para identificar os múltiplos padrões de caminhos. A partir desses critérios de agrupamentos, são obtidos os grupos que definem os diferentes caminhos dos sinais e as camadas neurais ativadas no circuito neural. Ao final deste capítulo, é apresentado o mapeamento geral do circuito neuromotor do gafanhoto com base nos resultados da máxima coerência da transferência de informação.

\subsection{Resultados das análises do STA para neurônios sensores}

Nesta seção, apresentam-se os principais resultados da análise das curvas de STA e as discussões envolvendo os significados neurofisiológicos dos diferentes tipos de respostas.

Para a geração do sinais do STA, utilizou-se o modelo matemático proposto na Seção 4.1.1. Adicionalmente, obtiveram-se as respostas de velocidade do STA, através da derivada, por equações de diferenças, da curva original do STA. A Figura 5.1 mostra ambas as respostas do STA, para a posição e a velocidade.

Observa-se a existência de dois padrões distintos, após a análise de todos os experimentos. Desta forma, podem-se levantar algumas hipóteses quanto à interpretação destes resultados. A principal diferença entres as Figuras 5.1(a) e (b) é a oposição de fases entre os sinais de posição do STA. Isso pode ser interpretado como diferentes tipos de resposta geradas no $\mathrm{FeCO}$.

Na Figura 5.1(a), o semiciclo da curva do STA anterior à ocorrência do spike encontra-se com valores positivos, o que indica valores médios relacionados à depolarização do neurônio. Por outro lado, na Figura 5.1(b), o semiciclo anterior do STA encontra-se com valores negativos, indicando uma tendência para o processo de inibição do neurônio.

Com base nessas evidências, sabe-se que o $\mathrm{FeCO}$ é constituído por um feixe de neurônios sensíveis aos movimentos do apódema. Dessa forma, ao se coletarem os sinais, dependendo 


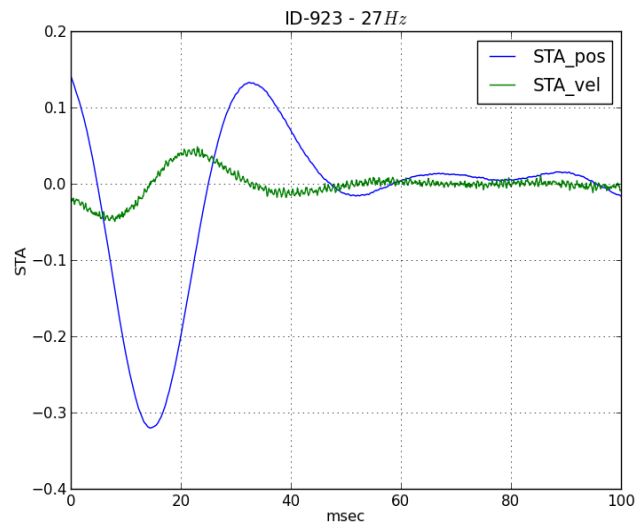

(a)

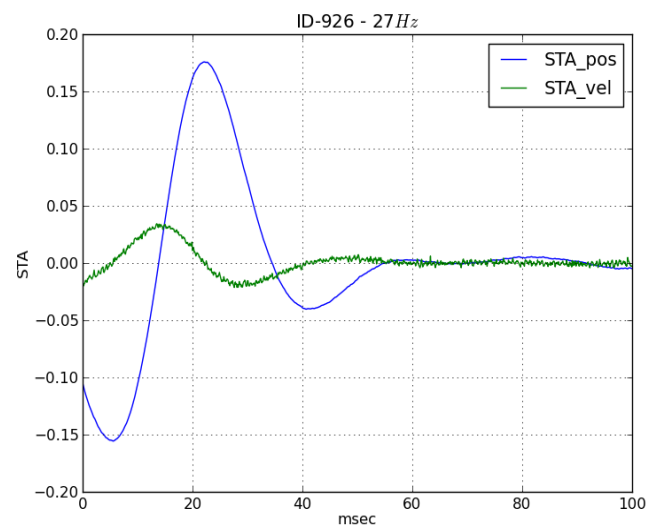

(b)

Figura 5.1: Resultados das curvas médias dadas pelo análise de STA. Curvas características de primeira e segunda ordem, relacionadas à interpretação do controle motor de posição (em azul) e velocidade (em verde), respectivamente. A principal característica entre (a) e (b) é a oposição de fases entre os sinais. Em (a) o semiciclo da curva do STA anterior à ocorrência do spike encontra-se com valores positivos, indicando depolarização do neurônio. Em (b) o semiciclo anterior do STA possui valores negativos, tendência para a inibição do neurônio.

do neurônio pinçado, pode-se, de fato, obter diferentes tipos de respostas neurais, a partir da mesma estrutura neural, conforme é descrito em Burrows (1996).

Assim, analisando-se os dois comportamentos distintos do $\mathrm{FeCO}$, observa-se que, em um padrão, ocorre uma resposta à sensibilidade do movimento. E, no outro padrão, ocorre a resposta à aplicação de força e à posição dos músculos. Para ilustrar essas hipóteses, obtiveram-se os resultados das dependências condicionais das $p d f$ 's, conforme apresentado na Figura 5.2.

A Figura 5.2(a) mostra o resultado relacionado à resposta dinâmica do $\mathrm{FeCO}$. Isso é ilustrado pela $p d f, p($ stim $\mid$ spike $)$, que possui valores concentrados em torno do ponto central da posição, indicando que é mais sensível ao movimento.

Já na Figura 5.2(b) ocorre o oposto: os valores da $p d f, p($ stim|spike), estão espalhados e concentrados nos extremos da distribuição. Mostrando fortes indícios de que nestes experimentos o $\mathrm{FeCO}$ foi mais sensível a posição. Em termos práticos, para a neurofisiologia do inseto, isso pode estar relacionado à aplicação de força muscular e à posição do membro.

A principal evidência de que o $\mathrm{FeCO}$ é sensível ao movimento e à aplicação de força é o modelo de fusos musculares encontrados em mamíferos, conforme visto em Fouad et al. (2003). No entanto, mais estudos são necessários para que se consolidem essas evidências sobre a capacidade sensorial do $\mathrm{FeCO}$ de gafanhotos. 

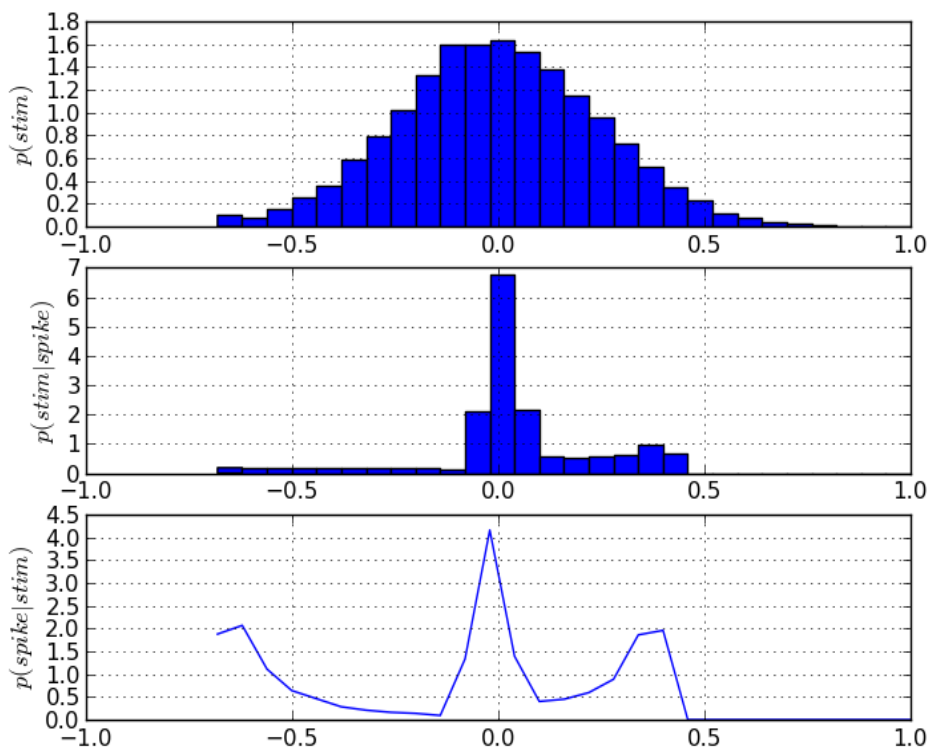

(a)
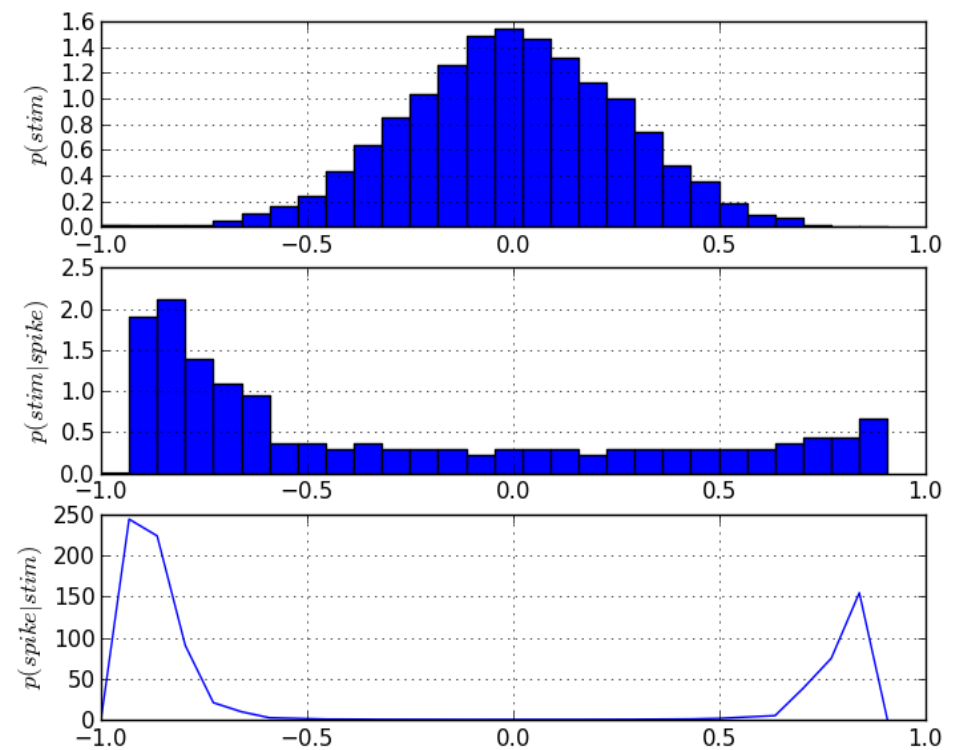

(b)

Figura 5.2: Funções densidade de probabilidades dos sinais de excitação e spikes do $\mathrm{FeCO}$ vindas da análise de STA. (a) Valores concentrados na região central da distribução - hipótese levantada: sensibilidade ao movimento. (b) Valores espalhados ao longo da distribuição e concentrados nas extremidades - sensibilidade de posição e força. 
Utilizando-se a correlação cruzada entre o estímulo e o $\mathrm{FeCO}$, observa-se similaridade com o resultado do $S T A$, para valores de $t<0$, conforme pode ser visto nas Figuras 5.3 e 5.4.

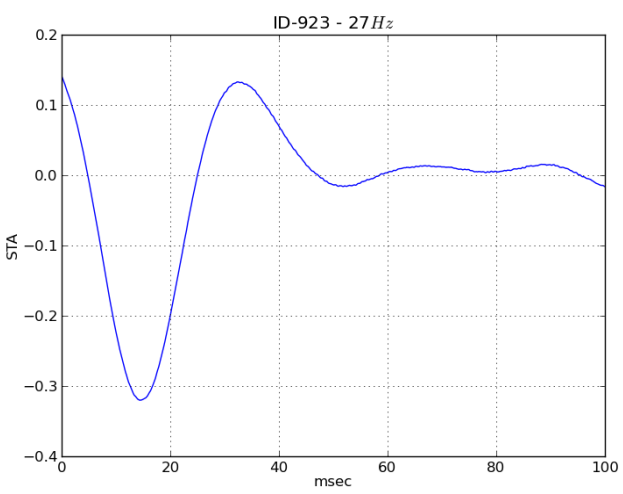

(a)

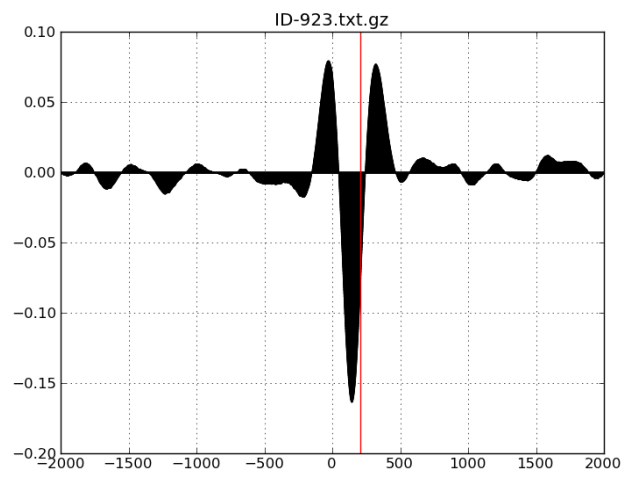

(b)

Figura 5.3: Comparação entre o STA e a correlação cruzada no modelo de neurônio sensor sensível ao movimento.

Ambos os resultados dessas figuras mostram o efeito de primeira ordem do STA ao comparálas com as respostas de correlação cruzada. Isso é considerado uma forma de validar os resultados dos STA mediante este tipo de estudo comparado.

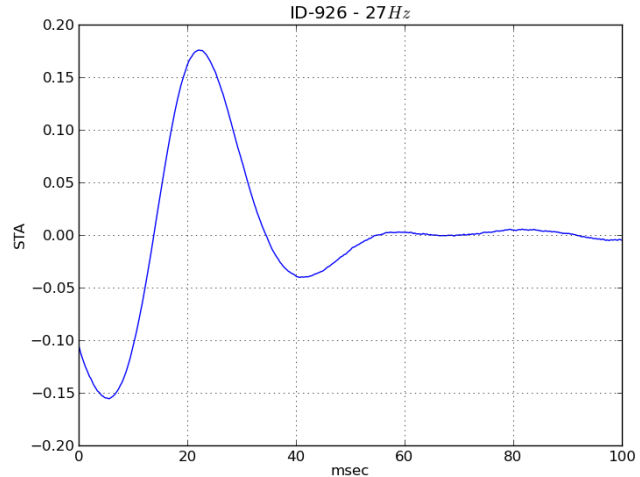

(a)

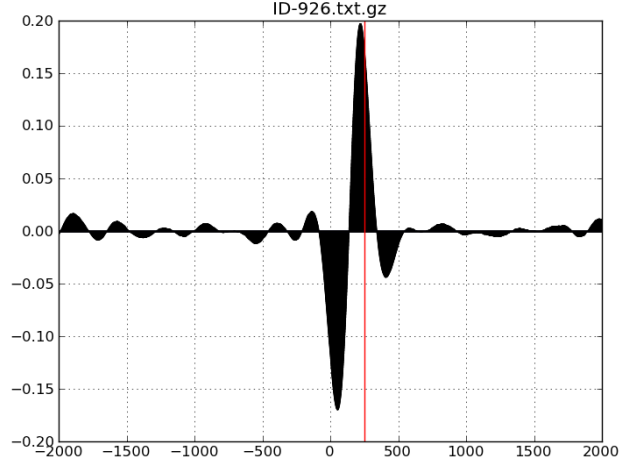

(b)

Figura 5.4: Comparação entre o STA e a correlação cruzada no modelo de neurônio sensor sensível à posição e aplicação de força. 


\subsection{Resultados dos dados de simulação para a transferência de in- formação}

Para discutir e avaliar os resultados dos dados simulados, são mostrados dois casos da $D M I$ e da $T E$ nas Figuras 5.5(a) e 5.5(b), respectivamente. Cada caso foi obtido a partir de diferentes valores da força de acoplamento, sendo $\zeta_{x y}=0,4$ e 1,0. Os resultados dos dados simulados mostram os erros de desvio introduzidos por diferentes tamanhos de amostras e forças de acoplamento.
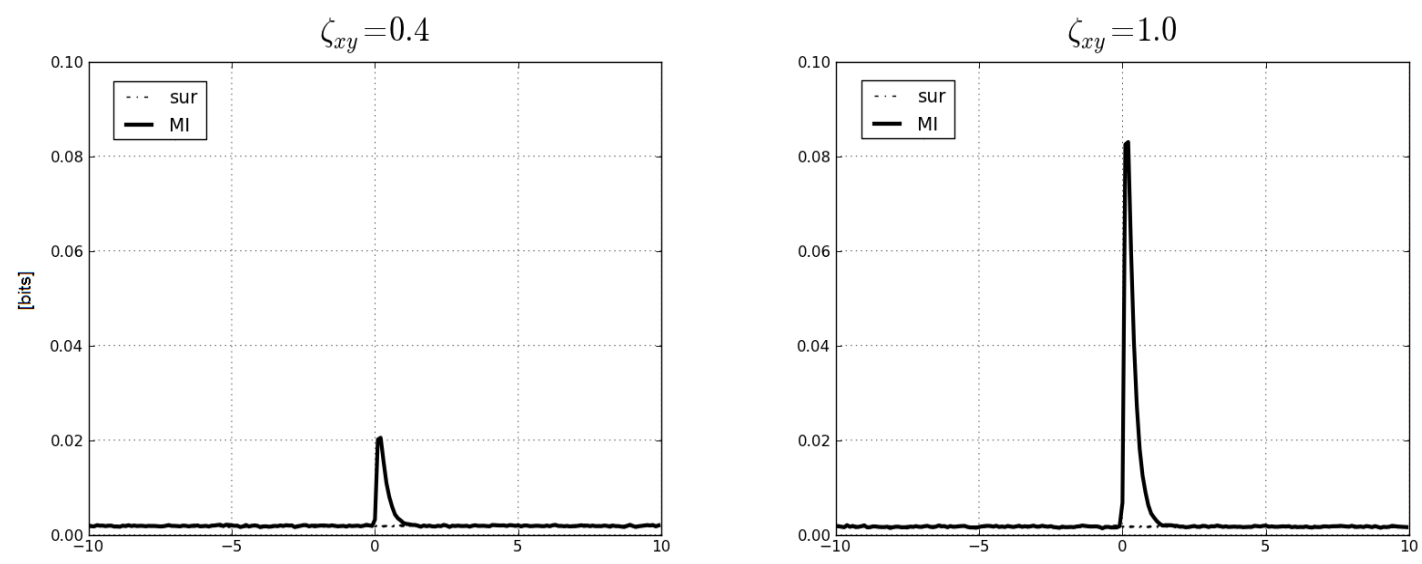

(a)
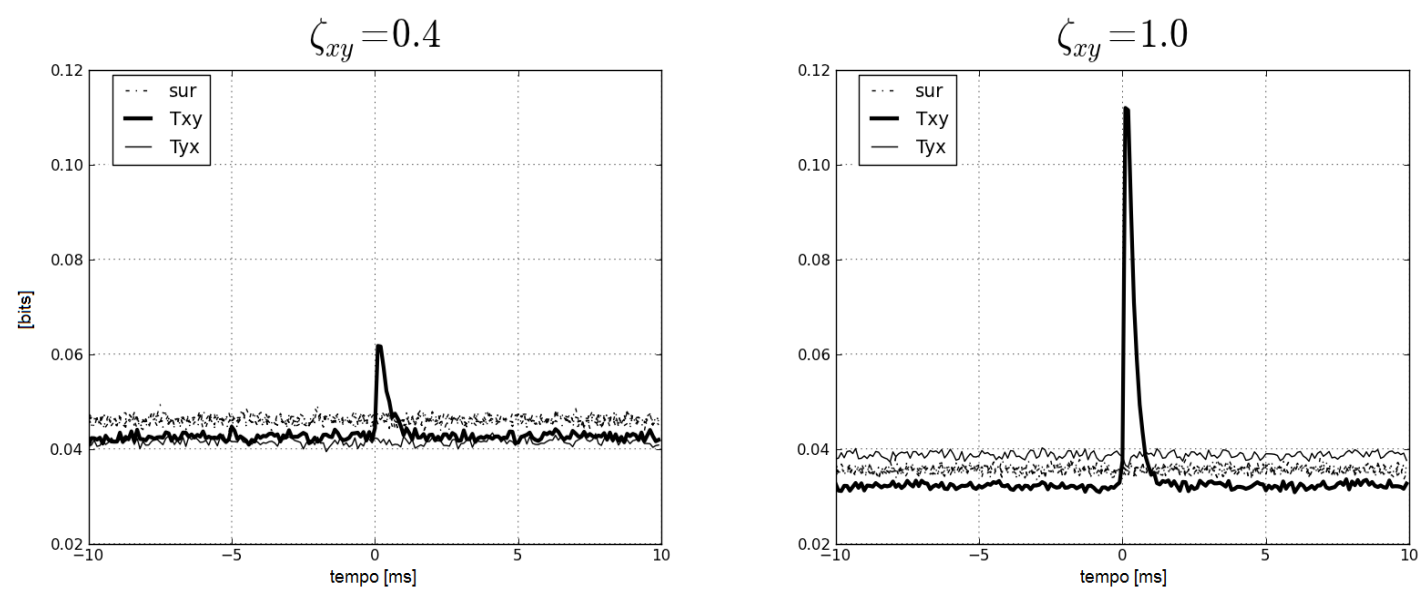

(b)

Figura 5.5: Análise de dados sintéticos para a (a) informação mútua atrasada e (b) a transferência de entropia com variações da força de conectividade a partir do modelo sintético proposto. $\mathrm{O}$ teste de conectividade utiliza valores para a força de acoplamento com $\zeta_{x y}=[0.4,1,0]$.

As curvas de resposta da informação mútua atrasada e da transferência de entropia são bastante semelhantes. No entanto, os níveis da linha de base sofrem flutuações nos seus res- 
pectivos valores. Esse efeito é observado com a utilização dos resultados a partir dos Surrogate Data. A Figura 5.5 mostra essas variações quando se comparam a DMI com a TE.

Os valores da linha de base são mais altos na $T E$, pois, devido à dimensão de sua $p d f$, o preenchimento dos dados acaba sofrendo mais variações quando comparada com a $p d f$ de segunda ordem da DMI. A atenção com os valores da linha de base é muito relevante para análises estatísticas, a fim de se evitar a obtenção de falsos positivos.

Utilizando-se as equações do Capítulo 3 sobre a normalização e compensação dos erros de desvio, obtiveram-se os resultados mostrados na Tabela 5.1. Os resultados do nível de transferência de informação, $\Delta \widehat{T E}$, para cada valor da força de associação da informação $\zeta_{x y}$, indicam que existe um maior nível de transferência de informação, quando $\zeta_{x y}=1,0$, validando as medidas de normalização e compensação.

Tabela 5.1: Valores máximos para a $D M I$ obtidos para os dados simulados.

\begin{tabular}{ccccc}
\hline \hline$\zeta_{x y}$ & $\sqrt{H(X) \cdot H(Y)}$ & $\hat{I}_{B}\left(X ; Y^{\tau}\right)$ & $\widehat{T E}_{B}(X \rightarrow Y)$ & $\Delta \widehat{T E}$ \\
\hline 0,4 & 3,57 & 0,53 & 0,45 & 0,151 \\
1,0 & 3,42 & 2,37 & 2,22 & 0,063 \\
\hline \hline
\end{tabular}

A Figura 5.6 apresenta os resultados com diferentes tamanhos de amostras e forças de associação da informação. Para melhor ilustrar a análise dos resultados, utilizou-se uma manipulação algébrica para o nível de transferência de informação, representado por $\psi_{\Delta}$, na Equação 5.1 ,

$$
\psi_{\Delta}=1-\Delta \widehat{T E}(X \rightarrow Y)
$$

Observa-se, a partir da Figura 5.6(a), que os valores do nível de transferência de informação, $\psi_{\Delta}$, aumentam de acordo com os diferentes tamanhos de amostras. Em oposição, os valores da $T E_{\text {sur }}$ apresentam um decaimento ao se aumentar os tamanhos das amostras, pois a dimensão da $p d f$ terceira ordem, $p\left(y_{n-\tau}, y_{n-\tau-k}, x_{n}\right)$, fica sujeita a maiores erros sistemáticos para amostras de tamanhos menores. Na Figura 5.6(b), é utilizado um mesmo tamanho de amostra $(10 \mathrm{seg})$ aplicado a diferentes valores da força de associação da informação para a $D M I$ e a $T E$ a fim de ilustrar essas diferenças entre a $D M I$ e a $T E$.

Esses resultados, com a utilização dos Surrogate Data, mostram que a DMI está menos sujeita a variações da linha de base. Os erros de desvio propagados, tanto na DMI quanto 


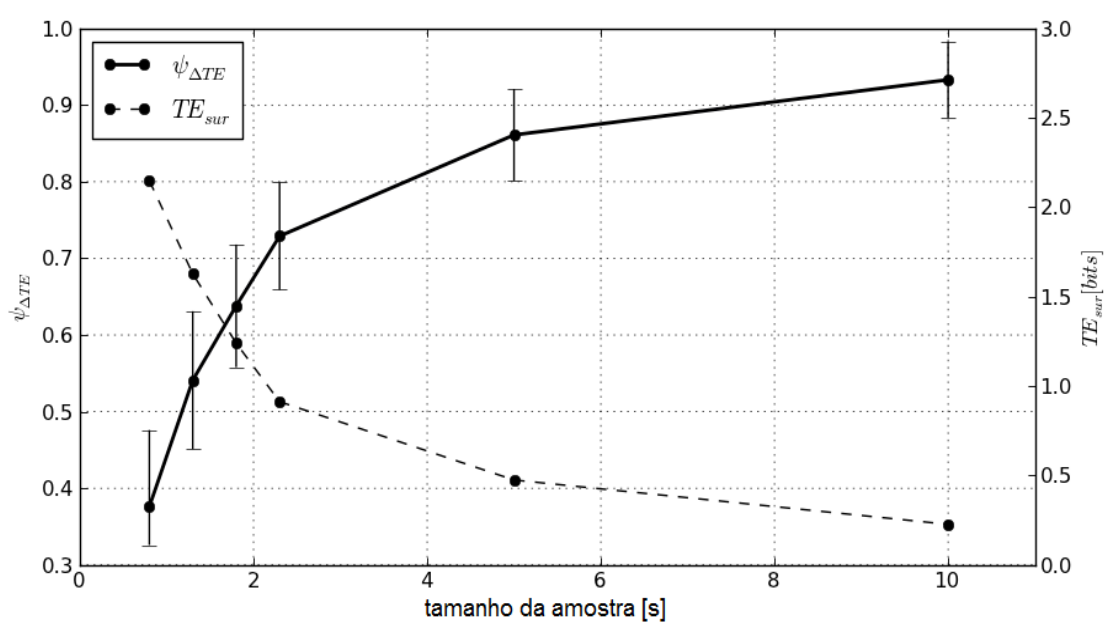

(a)

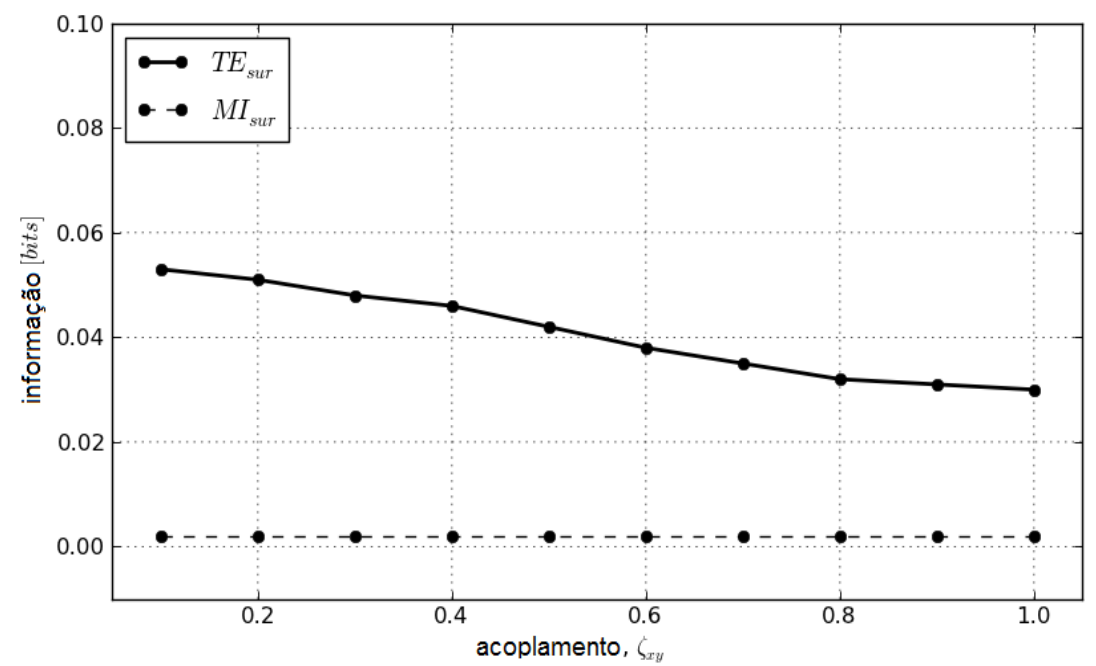

(b)

Figura 5.6: Análise dos dados simulados para a TE e a DMI. Análise simulada do nível de significância da transferência de informação normalizada e dos valores da transferência de entropia. Os dados simulados são baseados em dois processos estocásticos multivariados não lineares, $X_{t}$ e $Y_{t}$. (a) Os valores do nível de transferência de informação (linha contínua) são obtidos a partir de 10 séries temporais com dados simulados para cada tamanho de amostra. Todas as séries utilizaram o mesmo valor do nível nível de associação da informação, $\zeta_{x y}=0,90$. Os valores médios da transferência de entropia com os Surrogate Data (linha tracejada) mostram valores elevados da linha de base para amostras de tamanhos menores. (b) A TE e DMI, a partir do Surrogate Data, são estimadas utilizando-se 10 séries temporais para diferentes valores de associação. Utilizou-se o mesmo valor do tamanho da amostra (10 segundos de tempo de amostragem, com $F_{s}=10 \mathrm{kHz}$ ) para comparar o nível de informação Surrogate Data $\left(T_{\text {sur }}\right.$ e $M I_{\text {sur }}$ ) e diferentes valores de associação. 
na $T E$, têm grande influência sobre as medidas de transferência de informação. No entanto, apesar de a $T E$ ser mais efetiva para medidas da direção de transferência de informação, a $D M I$ apresenta resultados menos susceptíveis a esses erros provocados por amostras de tamanhos diferentes ou pela presença de ruídos nos sinais transmitidos. O que significa que, em alguns casos, a $D M I$ tem boa vantagem sobre a $T E$.

\subsection{Resultados dos dados reais para análise de conectividade do sistema neuromotor de insetos}

Foram realizados os testes e análises com os dados biológicos dos insetos. A análise se dividiu de acordo com os tipos de neurônios e suas respectivas funções fisiológicas nos circuitos neurais do sistema neuromotor do inseto.

\subsubsection{Neurônios sensores}

Com base na análise de cerca de 20 experimentos, foram obtidos os resultados dos valores de máxima coerência de informação para os neurônios sensores. Os resultados mostram os tempos de atraso entre: $7,5<\tau<28 \mathrm{~ms}$. Eles baseiam-se na $D M I$ e $T E$ aplicada nos sinais neurofisiológicos disponíveis no banco de dados dos experimentos. A Figura 5.7 mostra um exemplo de sinais analisados e sua respectiva resposta da DMI.

Conforme observado na Figura 5.7, verifica-se que existe, tipicamente, um padrão de tempo de atraso. Dessa forma, o valor médio dos tempos dessa resposta-padrão é caracterizada por um pico de máxima coerência, indicando a existência de uma única conexão neural. Pois, do ponto de vista anatômico, é o que se espera entre o estímulo do apódema e o $\mathrm{FeCO}$.

No entanto, observou-se a ocorrência de um pico central ou de até dois picos secundários em diferentes experimentos, conforme observado na Figura 5.8(b). A análise sobre a ocorrência de múltiplos picos no $\mathrm{FeCO}$ é discutida no final desta seção. Pois encontraram-se evidências de diferentes comportamentos funcionais dentro da mesma estrutura neural do $\mathrm{Fe} \mathrm{CO}$.

Como o padrão de respostas se manteve entre $7.5 \mathrm{~ms} \leq \tau \leq 28 \mathrm{~ms}$, caracterizando o padrão de uma única conexão sináptica, considerou-se, para a análise de múltiplos padrões de caminho, apenas o valor médio para $\tau=20 \mathrm{~ms}$. Na Tabela 5.2 são apresentados os resultados do atraso da máxima coerência da informação, para cada ID, obtida através da $D M I$ com seus 
(a)
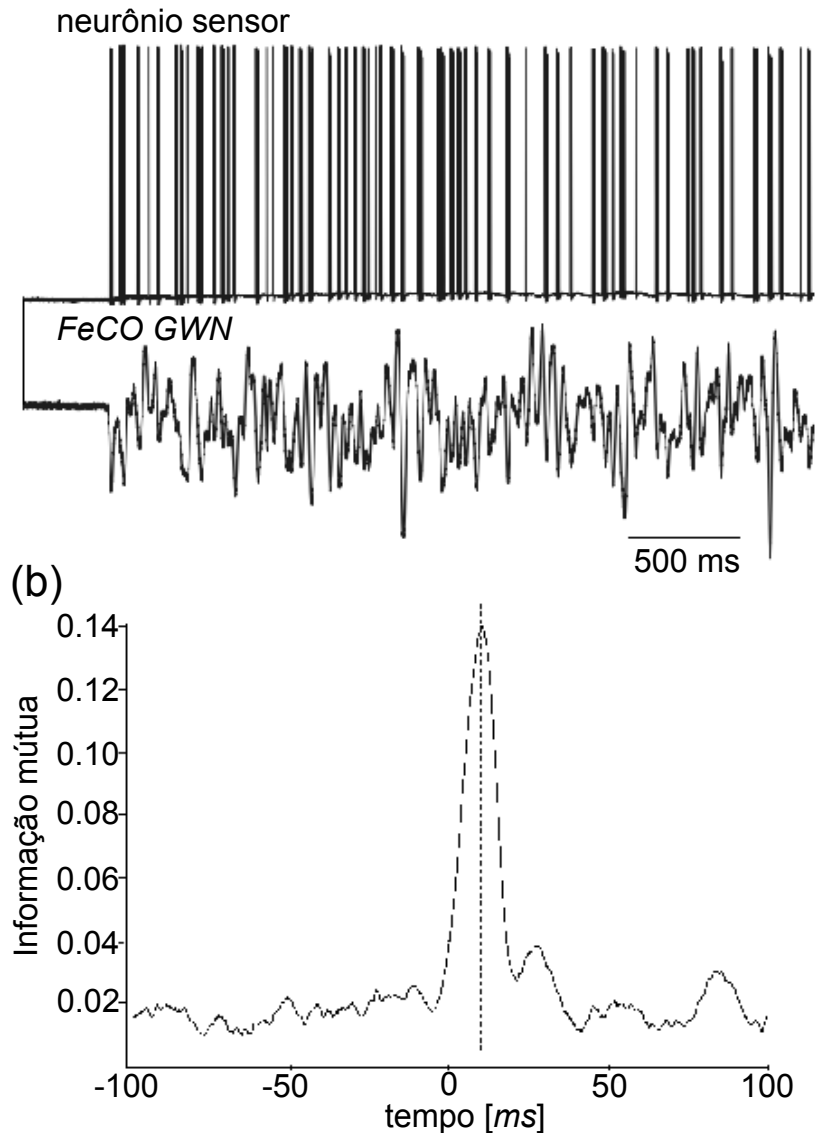

Figura 5.7: Padrão de resultado para a obtenção da curva de informação mútua atrasada para os neurônios sensores. (a) Trecho de gravação dos dados intracelulares da atividade neural ao se iniciar a ação do estímulo sobre o neurônio sensor. O apódema do $\mathrm{FeCO}$ é excitado com sinal GWN com largura de banda de frequência em $27 \mathrm{~Hz}$. (b) Um exemplo de uma curva característica de atraso da informação mútua retardada no neurônio sensor, com tempo de pico em $11 \mathrm{~ms}$.

respectivos valores de tempo e níveis de ruído.

Para validação e comparação dos resultados, utilizou-se o algoritmo para a geração de 30 Surrogate Data para cada experimento. A diferença entre a informação mútua atrasada máxima e o valor médio obtido com o Surrogate Data (linhas de base nas Figuras 5.8 e 5.9) ilustra as relações dos níveis de significância entre os picos de máxima coerência e as linhas de base.

Na Figura 5.9 são mostrados os padrões de resposta para transferência de entropia. Os resultados obtidos mostram os atrasos de máxima coerência da informação, através da $T E$ de $X \rightarrow Y$ e $Y \rightarrow X$, além de serem revelados os resultados com os Surrogate Data. As curvas com os Surrogate Data sofrem flutuações entre os experimentos; isso acontece devido à ocorrência de diferentes tamanhos das amostras selecionadas a partir do banco de dados 


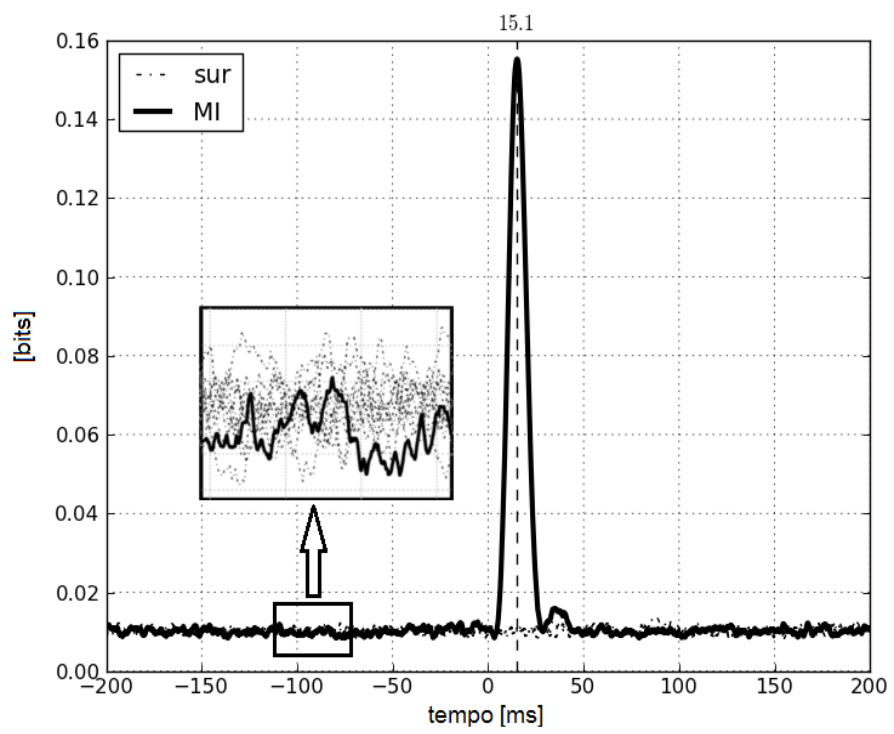

(a)

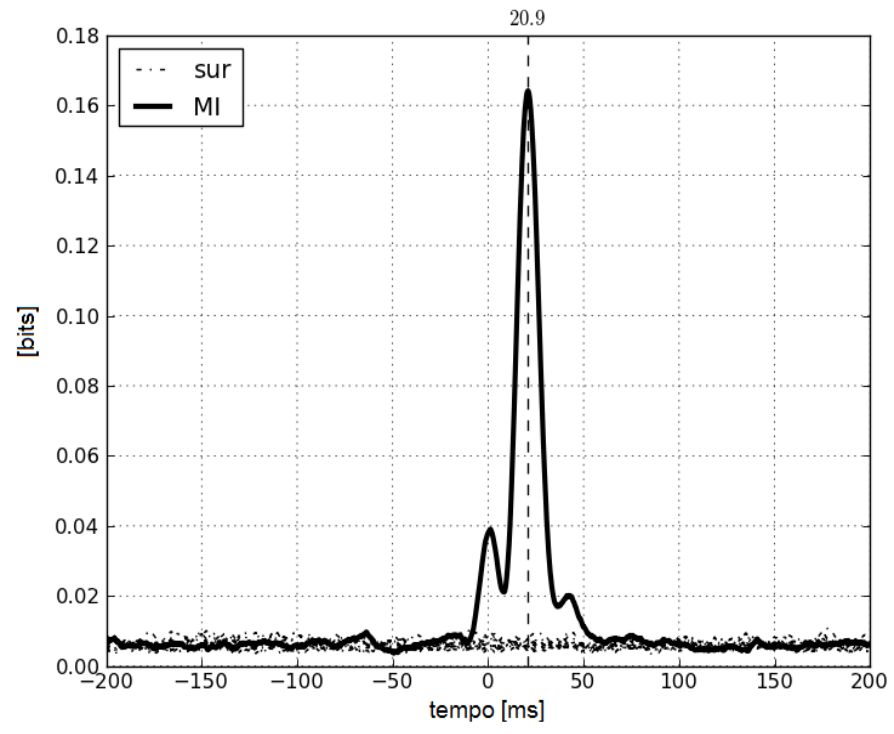

(b)

Figura 5.8: Curvas de transferência de informação pela $D M I$ entre a excitação de $27 \mathrm{~Hz}$ e um neurônio sensor. (a) Resposta característica de máxima coerência, em $15.1 \mathrm{~ms}$ da informação entre a excitação e os sinais do neurônio sensor. Os níveis da linha de base se mantêm constantes, mas diferentes de zero, conforme mostrado no detalhe. (b) Resposta de coerência de informação (ID-923) com picos secundários, devido à presença de sinais arbitrários vindos de outros neurônios durante a coleta dos dados. Para a análise geral dos dados, é considerado apenas o pico de máxima coerência em $20,9 \mathrm{~ms}$ 
Tabela 5.2: Valores médios das máximas informações mútuas para os neurônios sensores $\left[10^{-2}\right.$ bits].

\begin{tabular}{|c|c|c|c|}
\hline ID & BW[Hz] & Tempo[ms] - (DMI) $\left[10^{-2}\right.$ Bits $]$ & Ruído $\left[10^{-2}\right.$ Bits $]$ \\
\hline ID-922* & 27 & - & $\overline{2,6}$ \\
\hline ID-923 & 27 & $20,9-(16,4)$ & 0,6 \\
\hline ID-926 & 27 & $25,0-(10,1)$ & 0,4 \\
\hline ID-929 & 27 & $21,5-(18,7)$ & 0,8 \\
\hline ID-932 & 27 & $21,7-(27,1)$ & 0,9 \\
\hline ID-934 & 27 & $22,0-(10,4)$ & 0,4 \\
\hline ID-937 & 27 & $17,3-(7,0)$ & 0,6 \\
\hline ID-939 & 27 & $11,8-(14,1)$ & 0,9 \\
\hline ID-942 & 27 & $20,0-(7,0)$ & 0,5 \\
\hline ID-945 & 27 & $8,4-(10,0)$ & 0,1 \\
\hline ID-948 & 27 & $10,8-(6,6)$ & 0,2 \\
\hline ID-1001* & 27 & $4,0-(4,6)$ & 0,1 \\
\hline ID-1004 & 27 & $12,8-(9,7)$ & 0,4 \\
\hline ID-1006 & 27 & $27,8-(7,3)$ & 0,7 \\
\hline ID-1008 & 27 & $18,6-(6,7)$ & 0,5 \\
\hline ID-1010 & 27 & $18,5-(8,8)$ & 0,2 \\
\hline ID-1013 & 27 & $7,9-(5,7)$ & 0,4 \\
\hline ID-1016 & 27 & $18,9-(9,1)$ & 2,3 \\
\hline ID-1019 & 27 & $20,8-(10,0)$ & 0,5 \\
\hline \multicolumn{2}{|c|}{ Valores médios dos dados selecionados $($ sem *) } & $17,92-(10,9)$ & $\overline{0,4}$ \\
\hline & Valores médios de todos os dados & $17,15-(10,5)$ & 0,4 \\
\hline
\end{tabular}

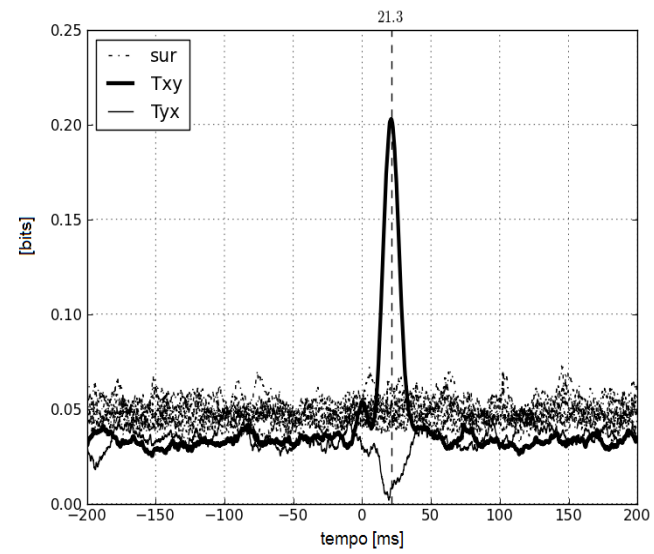

(a)

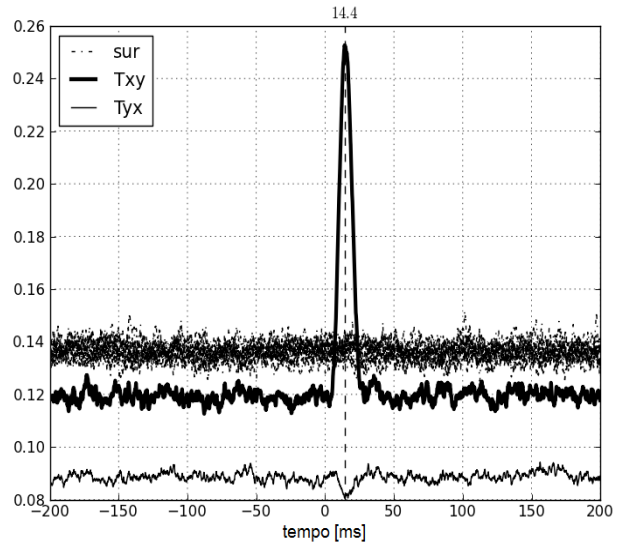

(b)

Figura 5.9: Transferência de entropia entre a excitação $27 \mathrm{~Hz}$ e um neurônio sensor. (a) Dados obtidos da ID-923 com um tamanho de amostra de 38s. (b) Dados obtidos da ID-945 com um tamanho de amostra de $20 \mathrm{~s}$. 
do experimento. No caso da Figura 5.9(a), os dados foram obtidos da ID-923, da qual foi selecionado um tamanho de amostra de $38 s$ e, na Figura 5.9(b) da ID-945, um tamanho de amostra de $20 s$.

\section{Análise das hipóteses para as características funcionais dos neurônios sensores do $\mathrm{FeCO}$}

Observaram-se pequenas variações dos tempos de atraso entre os próprios neurônios sensores. No entanto, quando se considera apenas o valor médio em si, o tempo de atraso dos neurônios está relacionado a um único padrão de caminho. Por outro lado, ao se analisar todos os resultados dos neurônios sensores entre si, os resultados obtidos pela DMI apontam para algumas evidências de comportamentos distintos dentro da própria estrutura do $\mathrm{FeCO}$.

Desta forma, também foram identificados dois padrões de comportamento do $\mathrm{FeCO}$, conforme observado nas Figuras 5.10(a) e (b). Isso ocorre de forma similar aos dois tipos de padrões obtidos pela análise do STA, conforme descrito na Seção 5.1.

Nas Tabelas 5.3 e 5.4, são apresentados os dois grupos de tempos de atraso da transferência máxima de informação. Esses valores de tempo ilustram o comportamento que ocorre nas Figuras 5.10(a) e (b), respectivamente. As figuras podem ser consideradas como assinaturas características de cada uma das funções de um neurônio sensor do $\mathrm{FeCO}$.

Tabela 5.3: Valores dos picos centrais baseados no valor médio dos atrasos e da máxima informação mútua atrasada do neurônios do $\mathrm{FeCO}$.

\begin{tabular}{c|c|c|c}
\hline \hline ID & BW[Hz] & Pico central [ms] & Max DMI $\left[10^{-2}\right.$ Bits] \\
\hline \hline ID-923 & 27 & 20,9 & 16,4 \\
\hline ID-929 & 27 & 21,5 & 18,7 \\
\hline ID-932 & 27 & 21,7 & 27,1 \\
\hline ID-934 & 27 & 22,0 & 10,4 \\
\hline ID-937 & 27 & 17,3 & 7,0 \\
\hline ID-1008 & 27 & 18,6 & 6,7 \\
\hline ID-1010 & 27 & 18,5 & 8,8 \\
\hline ID-1016 & 27 & 18,9 & 9,1 \\
\hline ID-1019 & 27 & 20,8 & 10,0 \\
\hline \hline \multicolumn{2}{c}{ Valores médios } & $\mathbf{2 0 , 0 2}$ & $\mathbf{1 2 , 7}$ \\
\hline \hline
\end{tabular}

Conforme comentado anteriormente, os valores máximos e mínimos dos tempos de atraso, das Tabelas 5.3 e 5.4, condizem com o intervalo de tempo médio para um único padrão caminho. No entanto, revela-se uma importante evidência que aponta para comportamentos funcionais distintos, que podem se referir à sensibilidade de movimento ou da aplicação de força gerada na excitação do apódema e identificada pelos neurônios do $\mathrm{FeCO}$. 


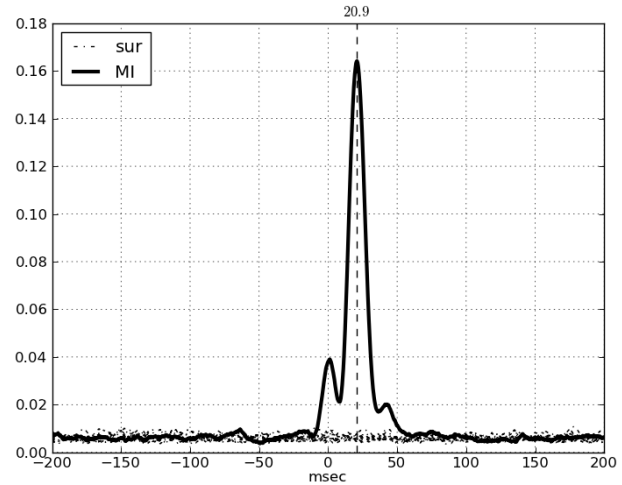

(a)

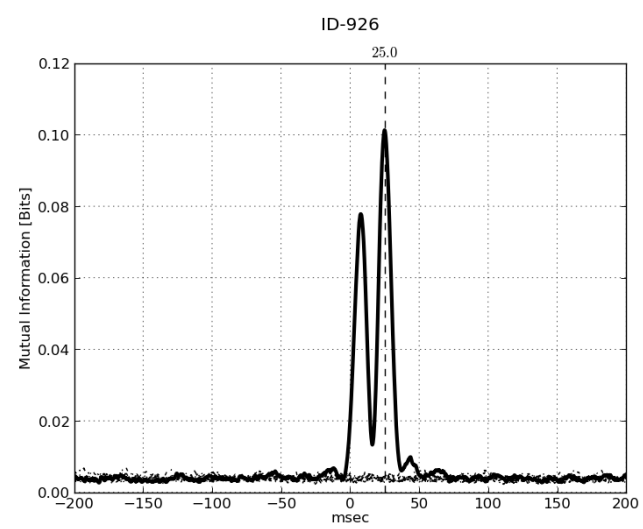

(b)

Figura 5.10: Resultados de diferentes padrões de atraso da transferência de informação nos neurônios sensores (a) Valores concentrados na região central da transferência para a hipótese de sensibilidade ao movimento. (b) Valores de picos duplos para a hipótese de sensibilidade de posição e força. (Foram utilizados os mesmos experimentos e os mesmos trechos das séries temporais dos resultados apresentados na análise do STA)

Tabela 5.4: Valores dos picos duplos baseados no valor médio dos atrasos e da máxima informação mútua atrasada do neurônios do $\mathrm{FeCO}$.

\begin{tabular}{c|c|c|c|c}
\hline \hline ID & BW[Hz] & Pico 1 [ms] & Pico 2 [ms] & Max DMI $\left[10^{-2}\right.$ Bits] \\
\hline \hline ID-926 & 27 & 11,9 & 25,0 & 10,1 \\
\hline ID-939 & 27 & 11,8 & 25,8 & 14,1 \\
\hline ID-945 & 27 & 8,4 & 24,9 & 10,0 \\
\hline ID-948 & 27 & 10,8 & 26,1 & 6,6 \\
\hline ID-1001 & 27 & 4,0 & 22.9 & 4,6 \\
\hline ID-1013 & 27 & 7,9 & 24,9 & 5,7 \\
\hline \multicolumn{6}{r|}{ Valores médios } & $\mathbf{9 , 1 3}$ & $\mathbf{2 4 , 9}$ & $\mathbf{8 , 5 1}$ \\
\hline
\end{tabular}




\subsubsection{Interneurônios spiking}

Os resultados baseiam-se na máxima coerência da transferência de informação entre as séries temporais a partir dos dados coletados dos interneurônios spiking dos insetos. Foram utilizados cerca de 25 experimentos realizados com os gafanhotos. Na Figura 5.11 são mostrados alguns resultados das curvas de atraso e máxima coerência da informação, obtida para os sinais dos interneurônios spiking. Para a comparação dos níveis estatísticos de significância, foram utilizados os Surrogate Data.

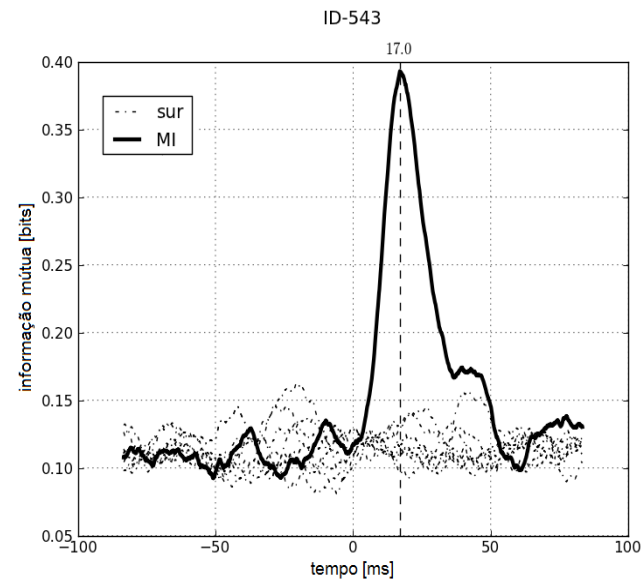

(a)

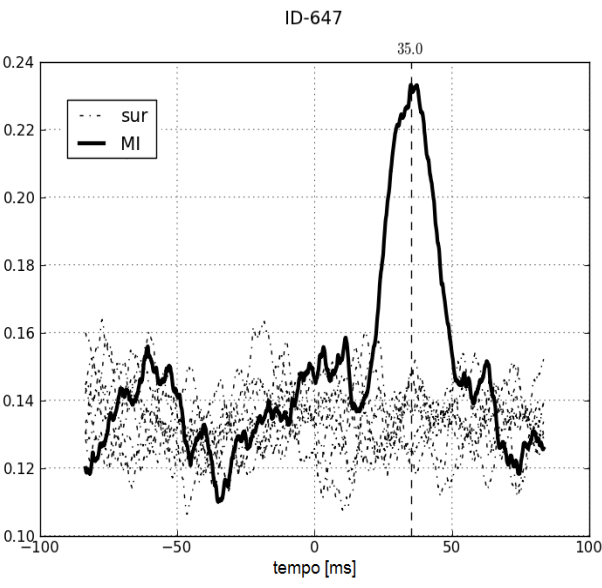

(b)

Figura 5.11: Respostas-padrão da informação mútua atrasada para sinais spiking. Observamse em (a) e (b) as assinaturas de cada padrão de múltiplo caminho, com tempos de atraso em torno de $17 \mathrm{~ms}$ e $35 \mathrm{~ms}$, respectivamente. A esses tempos estão relacionadas camadas neurais acessadas nos interneurônios do gânglio metatorácico.

Assim, utilizando-se os valores da informação mútua atrasada e dos tempos de atraso de cada experimento, montou-se o gráfico de distribuição dos tempos pelos valores da máxima coerência da transferência de informação, conforme é apresentado na Figura 5.12.

Na Figura 5.13 são apresentados os resultados de todas as análises dos interneurônios spiking. Os padrões de tempos para interneurônios spiking possuem curvas características, como visto na Figura 5.13(a). Os interneurônios com menores valores de informação mútua atrasada apresentaam as maiores capacidades de canal.

Para classificar-se o número de grupos $(\eta)$ de acordo com os múltiplos caminhos definidos pelos tempos de atraso e a $D M I$ máxima, utilizou-se o critério de agrupamentos definidos pelo BIC (Zak-Szatkowska e Bogdan, 2011). Esse critério busca um valor de agrupamento ótimo baseado na sua dispersão estatística, através das medidas de variância. As amostras são 


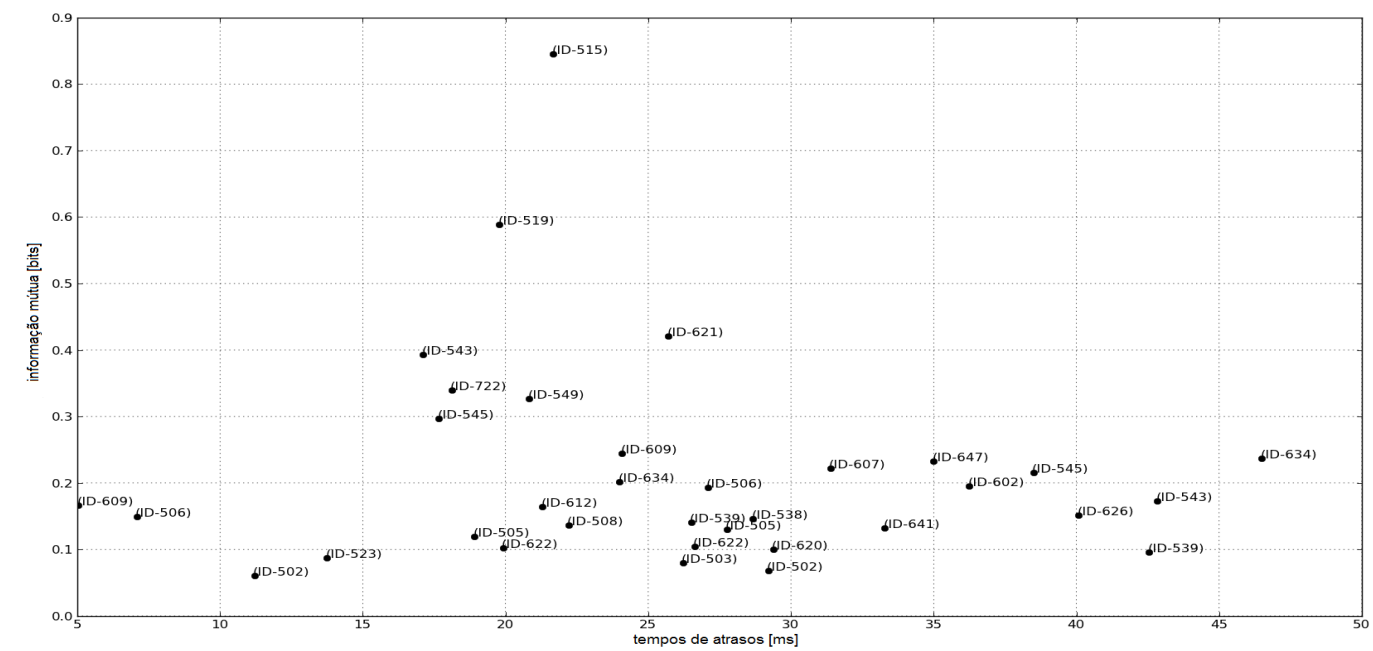

Figura 5.12: Distribuição dos tempos de atraso e suas respectivas informações mútuas para os interneurônios spiking. Este é um mapeamento dos tempos de atraso pelo máximo valor de informação mútua. Com base nesse mapa de pontos é aplicado o classificador bayesiano (BIC) para verificar os padrões de agrupamentos deste cluster.

definidas a partir de misturas de gaussianas 2-D de acordo com suas distribuições de ocorrência. São testados diferentes valores de agrupamento, com $2 \leq \eta \leq 6$. Para cada valor de $\eta$, é calculada a medida de dispersão dada pelo BIC, conforme é mostrado na Figura 5.14(a). O menor valor de dispersão encontrado determina o número de grupos (clusters) dentro do conjunto de dados. Baseado nessa ideia, o menor valor do BIC é 184.24, para $\eta=2$. Alguns dados do conjunto são considerados outliers, devido à sua discrepância com os valores médios dos agrupamentos.

Ao se definir o valor de $\eta$, encontram-se os grupos desejados conforme é apresentado na Figura 5.14. Dessa forma, são identificados os resultados esperados para os interneurônios spiking. Os agrupamentos obtidos pelo BIC indicam a existência de grupos de máxima coerência da informação. Os limites de tempo para os agrupamento estão dividos em dois intervalos, sendo $15 \leq \tau \leq 25 \mathrm{~ms}$ para o primeiro pico e $26 \mathrm{~ms} \leq \tau \leq 46 \mathrm{~ms}$ para o segundo. Com valores médios de transferência de informação em 0,38 e 0,16 , respectivamente, para os dois grupos.

Os resultados indicam o que realmente é esperado, pois, conforme Burrows (1996), para interneurônios spiking são esperados dois padrões de caminho no seu circuito neural. Esses interneurônios são responsáveis pela inibição dos neurônios motores do inseto, quando excitados pelo $\mathrm{FeCO}$. 


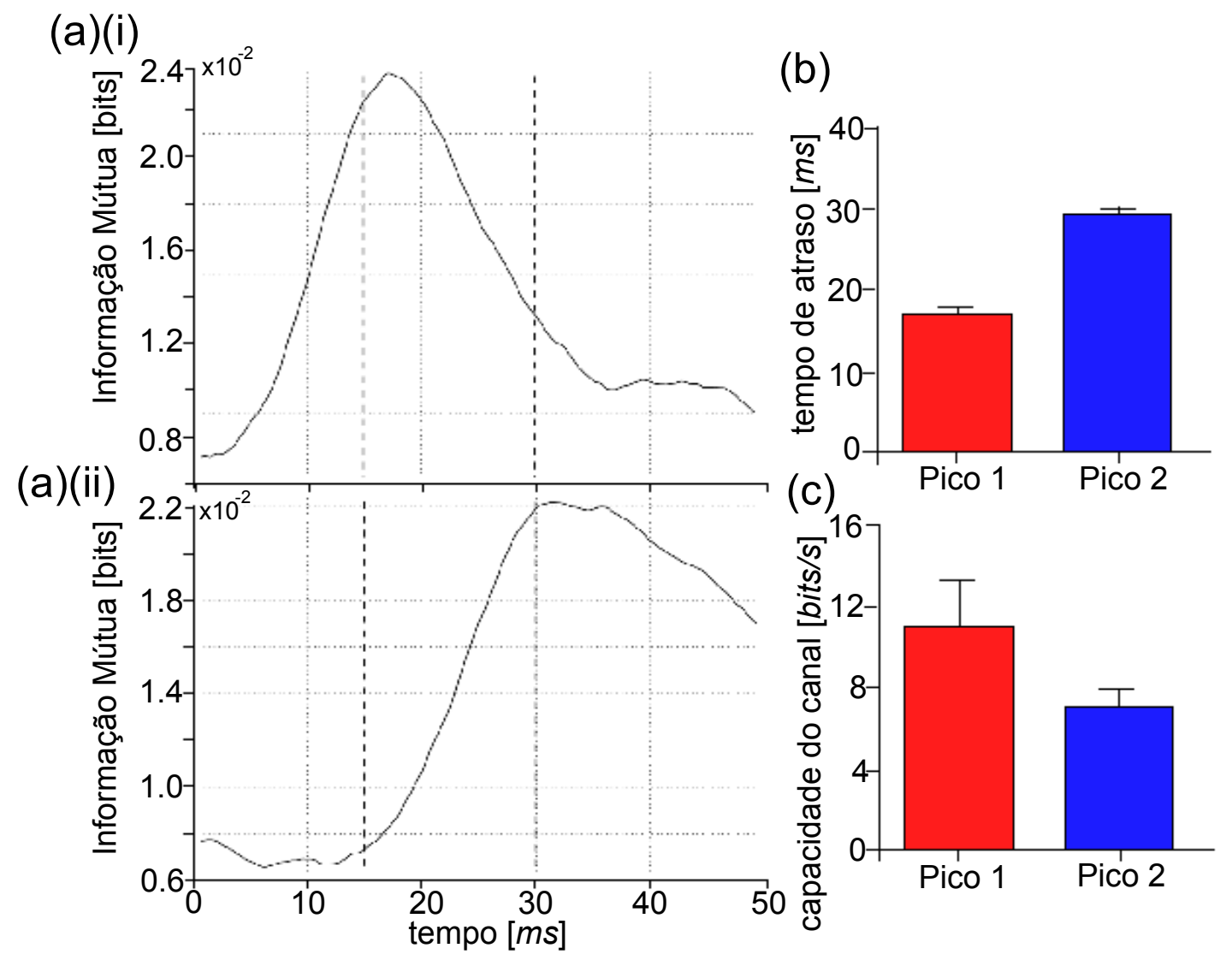

Figura 5.13: Resultados dos padrões de tempos para interneurônios spiking. (a)(i-ii) Exemplos de informação mútua atrasada apresentadas pelo exemplo de dois diferentes interneurônios. (b) Valores médios dos tempos de atraso para cada pico de máxima informação mútua atrasada, baseada na análise de 23 interneurônios spiking. (c) As capacidades de canal de cada grupo de interneurônios decaem com em relação ao aumento do tempo de atraso. Os valores foram estimados com base em uma largura de banda de transmissão de $350 \mathrm{~Hz}$. 


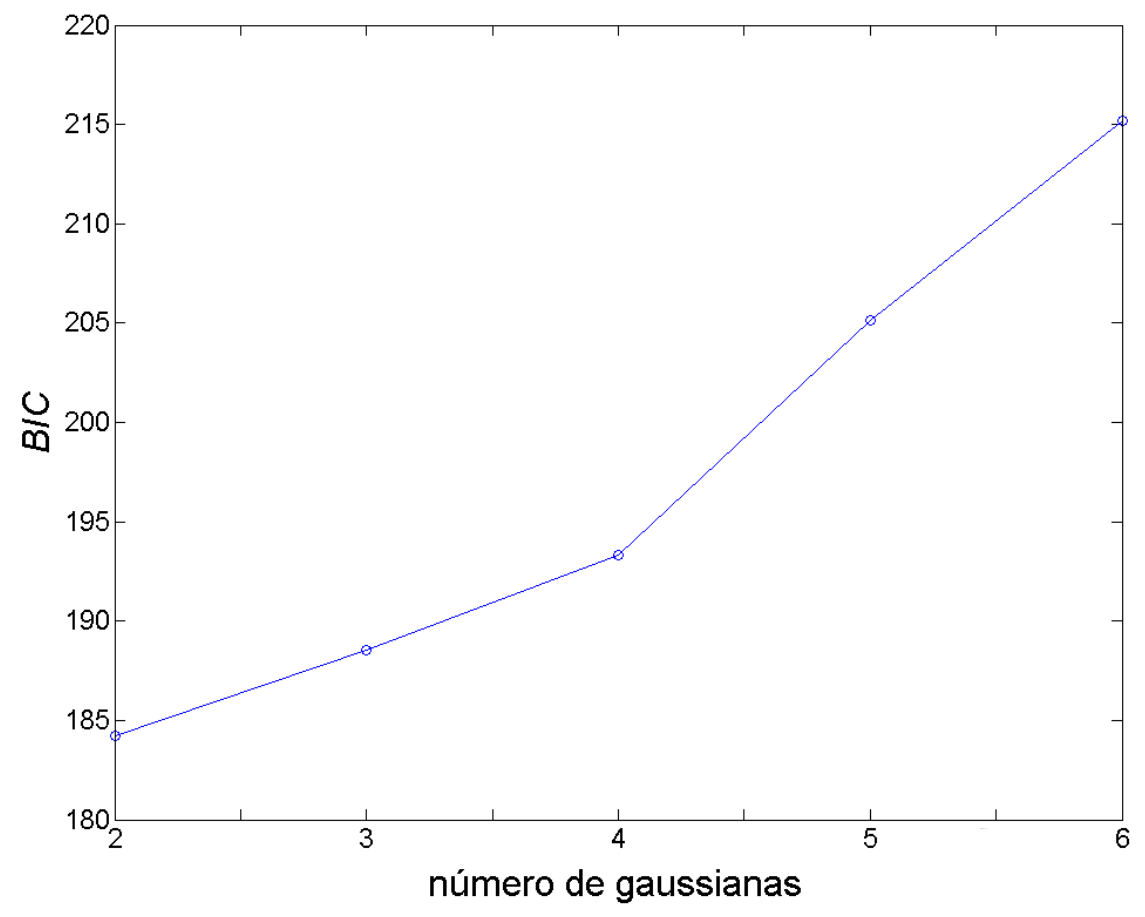

Figura 5.14: Clustering para os interneurônios spiking. Curva para o critério de escolha a fim de definir o número de mistura de gaussianas, sendo $\eta=[2,3,4,5,6]$. Critério para a definição de 2 agrupamentos baseado no valor mínimo de $B I C=184.24$.

\subsubsection{Interneurônios nonspiking}

A partir da análise de cerca de 30 experimentos de interneurônios nonspiking, foram obtidos resultados com os valores de atraso e máxima coerência da informação. Na Figura 5.16, aparecem as assinaturas típicas da DMI para esses sinais neurais. Para validação dos resultados, também, utilizaram-se os Surrogate Data. A diferença entre os valores de máxima coerência da informação e os valores das linhas de base obtidos com o Surrogate Data mostra a significância do nível de associação entre os sinais neurais.

A análise e discussão dos resultados sobre os interneurônios locais nonspiking têm alguma semelhança com interneurônios spiking; no entanto, a principal diferença entre esses dois tipos de sinais está nos padrões de múltiplos caminhos. Na Figura 5.17 são apresentados os resultados, a partir da aplicação dos métodos para obtenção da transferência de informação.

Utilizando os valores de $B I C$, o conjunto de dados são dipersos nas misturas gaussiana 2-D de acordo com sua distribuição de ocorrências. São testados os valores de agrupamento, com $2 \leq \eta \leq 6$, conforme é mostrado na Figura 5.18(a). O menor valor de dispersão encontrado é para $\eta=3, \operatorname{com} B I C=481.63$. Alguns dados do conjunto são considerados outliers, devido à 


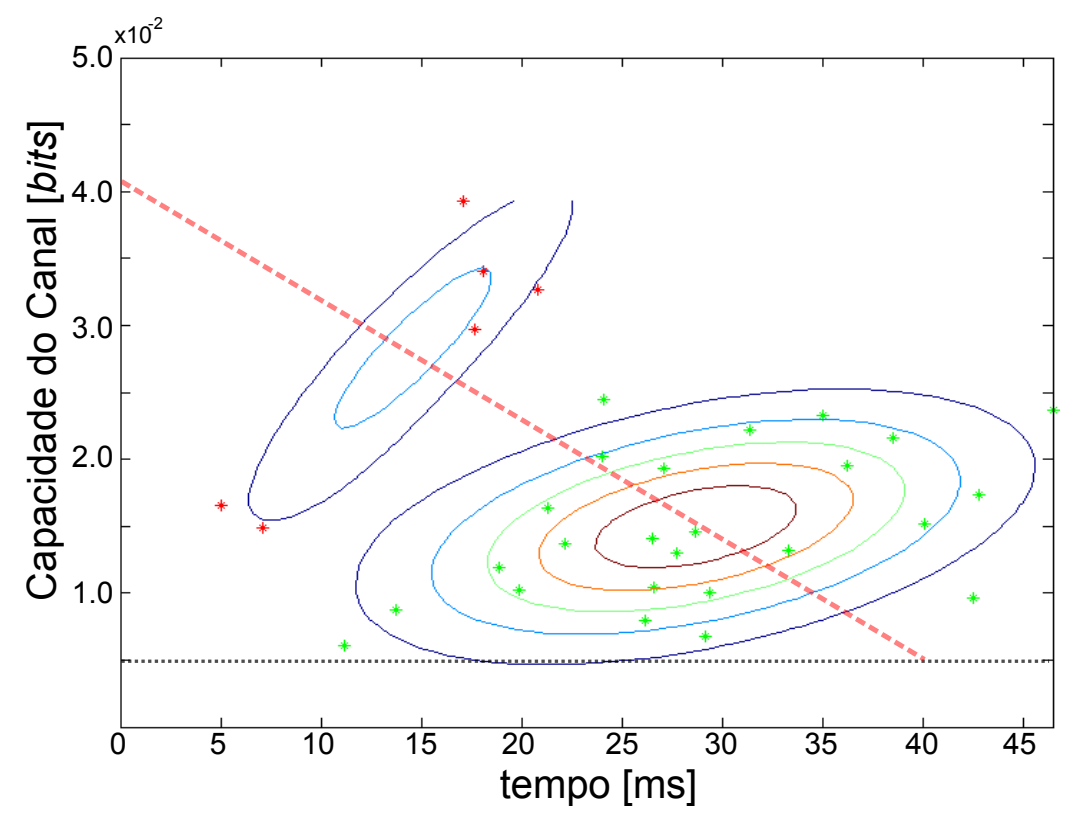

Figura 5.15: Clustering dos padrões de tempos para interneurônios spiking. Resultado do classificador bayesiano - BIC, em que são identificados 2 grupos distintos. Esses dois padrões estão relacionados às camadas neurais do gânglio metatorácico do gafanhoto. A linha tracejada vermelha representa do decaimento proporcional da informação mútua atrasada para os dois grupos. A linha pontilhada horizontal representa o valor médio da linha de base.

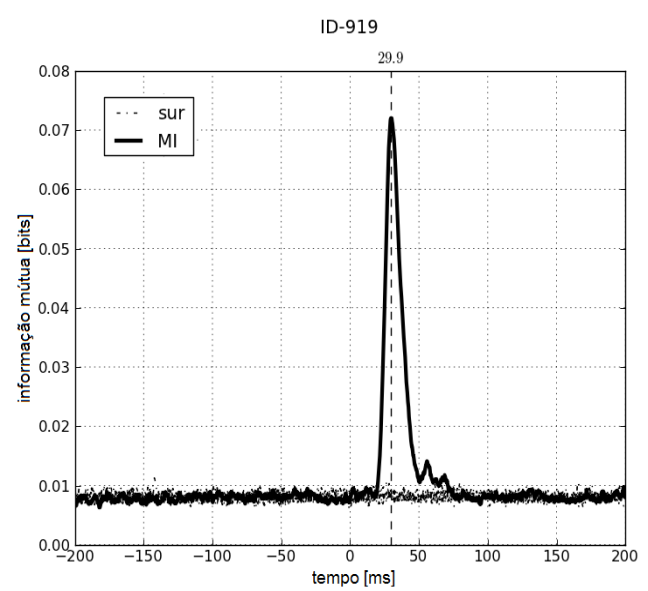

(a)

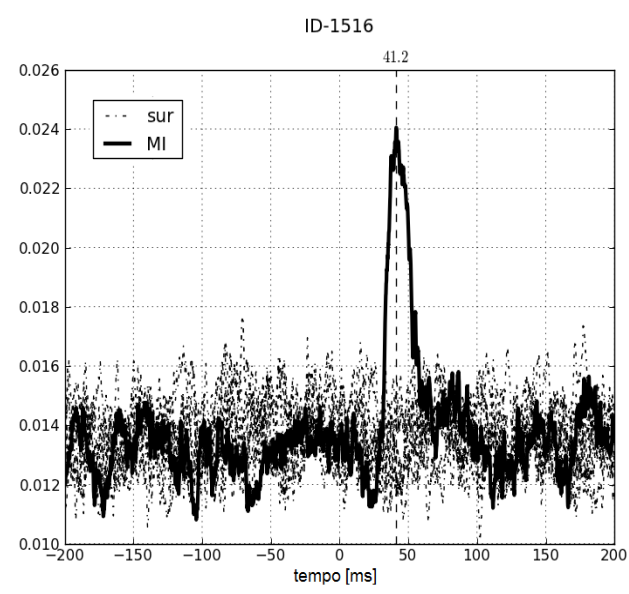

(b)

Figura 5.16: Respostas-padrão da informação mútua atrasada para sinais nonspiking. Observam-se em (a) e (b) as assinaturas de diferentes padrões caminho, com tempos de atraso em torno de 29, $9 \mathrm{~ms}$ e 41, $2 \mathrm{~ms}$, respectivamente. Cada conexão nas camadas neurais do gânglio possuem valor estimado em $15 \mathrm{~ms}$; assim, os valores dos tempos em (a) e (b), representam a identificação de 2 e 3 camadas respectivamente. 


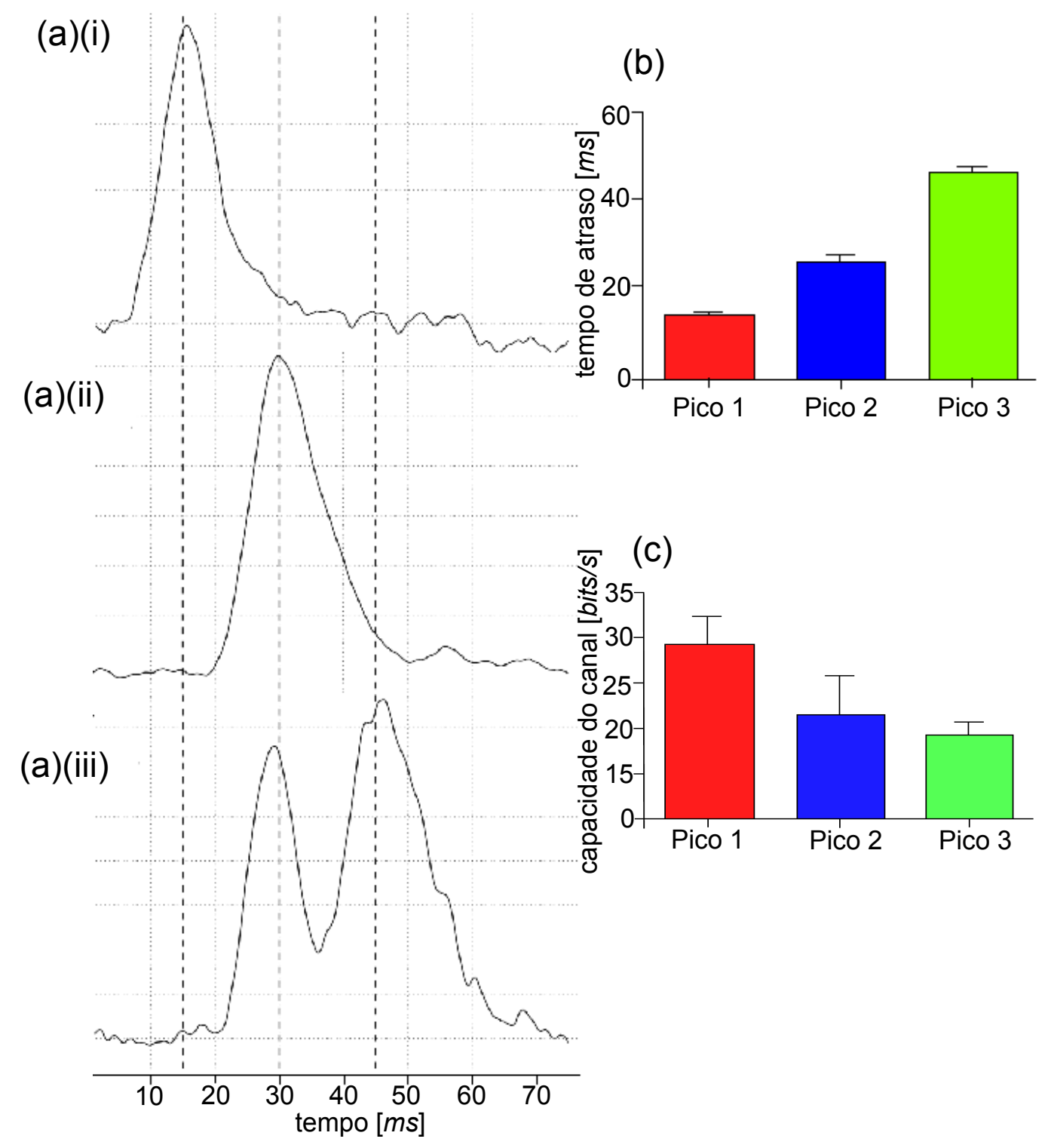

Figura 5.17: Resultados dos padrões de tempos para interneurônios nonspiking. (a)(i-iii) Exemplos das curvas da $D M I$ revelada a partir de três diferentes interneurônios . (b) Valores médios do pico de informação mútua atrasada, a partir de 24 interneurônios nonspiking. (c) Capacidades de canal de cada grupo de interneurônios, observa-se um decréscimo para valores maiores do tempo de atraso. Os valores foram estimados com base em uma largura de banda de transmissão de $350 H z$. 
sua discrepância com os valores médios dos agrupamentos.

Dessa forma, encontraram-se três grupos mostrados na Figura 5.18. Os resultados mostram os agrupamentos de acordo com os clusters obtidos pelo BIC. O primeiro grupo apresenta um intervalo de atraso em $11 \leq \tau<15 \mathrm{~ms}$, seguido pelo segundo grupo com intervalo em $25 \mathrm{~ms}<\tau<38 \mathrm{~ms}$ e pelo terceiro grupo com $41 \leq \tau \leq 49 \mathrm{~ms}$. Os valores médios da máxima coerência de informação são, respectivamente, 0, 08, 0, 06 e 0, 04, conforme a Figura 5.19.

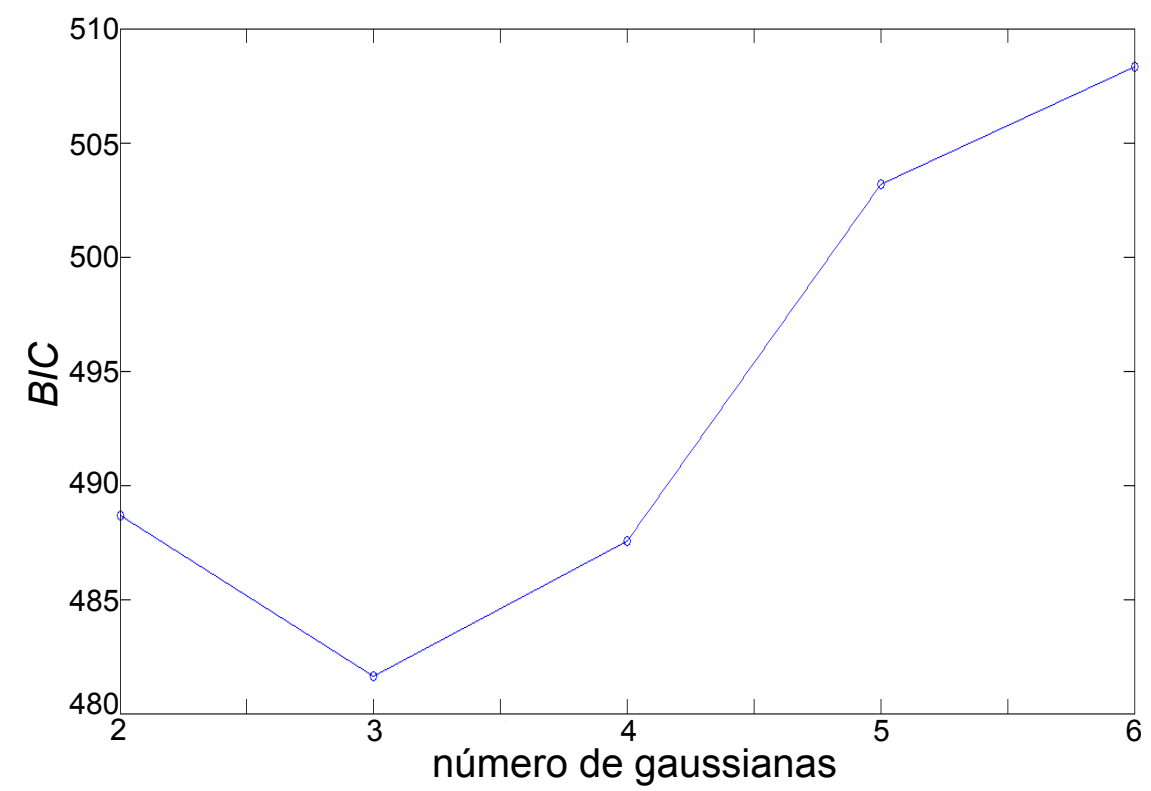

Figura 5.18: Clustering para interneurônios nonspiking. Curva $(B I C)$ para o critério de escolha a fim de definir o número de mistura de gaussianas, sendo $\eta=[2,3,4,5,6]$. Critério para definição de 3 agrupamentos baseado no valor mínimo de $B I C=481.63$.

Na Figura 5.19, a divisão dos dados em três grupos indica que os múltiplos padrões de caminhos nos interneurônios nonspiking condizem com a anatomia descrita por Burrows (1996). Observa-se que, para o menor tempo de atraso, ocorre o maior valor de transferência de informação, sendo que esse valor decresce para os dois outros tempos de atraso. Pois, de acordo com a topologia do circuito neural, o terceiro grupo representa uma conexão mais longa e indireta entre o neurônio sensor e o interneurônio nonspiking. Na Tabela 5.5, são apresentados os resultados do atraso da máxima coerência da informação para cada ID obtida através da $D M I$ com seus respectivos valores de tempo e níveis de ruído para os interneurônios nonspiking.

Com base nessas ideias e reunindo-se todas as informações obtidas com esses resultados, é apresentado, na próxima seção, um mapeamento geral do sistema neural do gânglio metatorácico do inseto. 
Tabela 5.5: Valores médios da DMI para os interneurônios nonspiking $\left[10^{-2}\right.$ bits $]$.

\begin{tabular}{|c|c|c|c|c|c|}
\hline ID & $\begin{array}{l}\text { BW } \\
{[\mathrm{Hz}]}\end{array}$ & $\begin{array}{c}\tau_{1}[\mathbf{m s}]- \\
(\boldsymbol{D M I})\left[10^{-2} \text { Bits }\right]\end{array}$ & $\begin{array}{c}\tau_{2}[\mathbf{m s}]- \\
(\boldsymbol{D M I})\left[10^{-2} \text { Bits }\right]\end{array}$ & $\begin{array}{c}\tau_{3}[\mathrm{~ms}]- \\
(\boldsymbol{D M I})\left[10^{-2} \text { Bits }\right]\end{array}$ & $\begin{array}{c}\text { Ruído } \\
{\left[10^{-2} \text { Bits }\right]}\end{array}$ \\
\hline ID-919 & 27 & & $29,9-(7,2)$ & & $\overline{0,8}$ \\
\hline ID-1038* & 27 & & $35,1-(3,1)$ & & 1,7 \\
\hline ID-1304* & 27 & & $36,3-(8,2)$ & & 5,5 \\
\hline ID-1339 & 27 & & $29,0-(11,9)$ & & 6,5 \\
\hline ID-1347 & 27 & $13,9-(9,7)$ & & $52,3-(12,2)$ & 6 \\
\hline ID-1348 & 27 & $13,5-(17,3)$ & & $47,8-(18,1)$ & 1,1 \\
\hline ID-1405* & 27 & $11,9 *-(2,0)$ & & & 1,5 \\
\hline ID-1410* & 27 & & & $46,1 *-(3,0)$ & 2 \\
\hline ID-1413* & 27 & & $30,7^{*}-(1,8)$ & $45,4^{*}-(1,7)$ & 1 \\
\hline ID-1419 & 27 & & $33,4-(5,5)$ & & 3,1 \\
\hline ID-1422 & 27 & & & $40,5-(2,5)$ & 0,9 \\
\hline ID-1424 & 27 & & $34,4-(7,4)$ & & 1,1 \\
\hline ID-1431 & 27 & & $35,6-(6,5)$ & & 2 \\
\hline ID-1434 & 27 & & $35,8-(8,3)$ & & 2,2 \\
\hline ID-1435 & 27 & & $36,9-(3,9)$ & & 1,6 \\
\hline ID-1436 & 27 & & $29,3-(4,8)$ & $46,2-(5,3)$ & 1,3 \\
\hline ID-1441 & 27 & & $37,4-(2,7)$ & $57,0-(1,7)$ & 1 \\
\hline ID-1442* & 27 & $14,8^{*}-(8,5)$ & $25,3^{*}-(9,9)$ & & 7,5 \\
\hline ID-1501 & 27 & & $31,5-(13,7)$ & & 3,9 \\
\hline ID-1505 & 27 & & $28,2-(6,1)$ & & 1,9 \\
\hline ID-1507 & 27 & & $27,6-(3,0)$ & $40,8-(2,6)$ & 1,7 \\
\hline ID-1508 & 27 & & $30,2-(4,9)$ & $54,5-(3,1)$ & 1,9 \\
\hline ID-1512 & 27 & & & $48,7-(6,5)$ & 2,6 \\
\hline ID-1516 & 27 & & & $41,2-(2,4)$ & 1,3 \\
\hline \multicolumn{2}{|c|}{ Médias dos dados (sem*) } & $13,1-(9,7)$ & $32,1-(6,2)$ & $\overline{477,4-(5,6)}$ & \\
\hline & lias totais & $13,5-(9,4)$ & $32,1-(6,4)$ & $47,3-(5,3)$ & \\
\hline
\end{tabular}

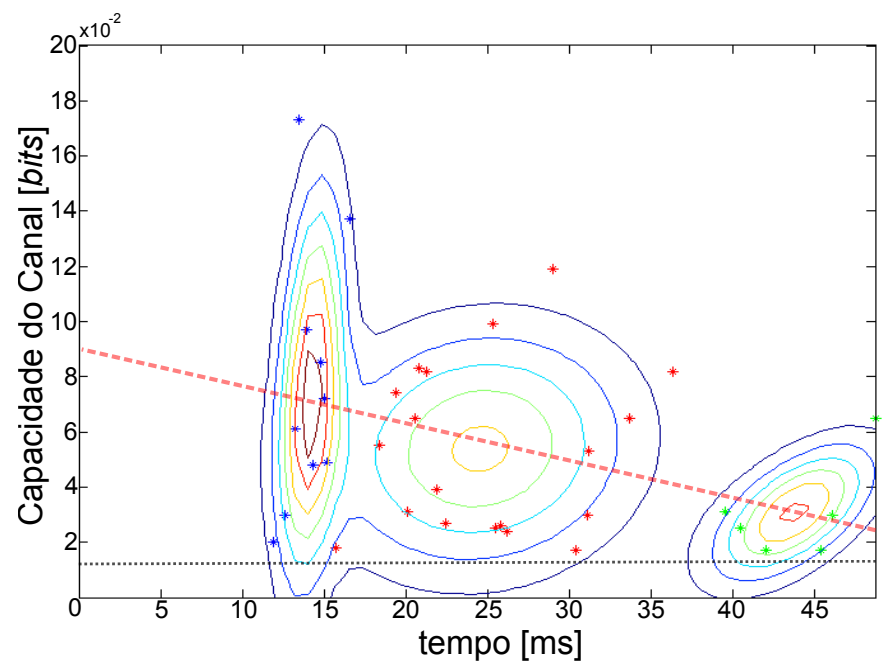

Figura 5.19: Clustering dos padrões de tempos para interneurônios nonspiking. Resultado do classificador bayesiano - BIC em que são identificados 3 grupos distintos. Em que estes 3 grupos identificados estão relacionados aos tempos de atraso médio de cada camada neural do gânglio metatorácico do gafanhoto. A linha tracejada vermelha representa do decaimento proporcional da informação mútua atrasada para os três grupos. A linha pontilhada horizontal representa o valor médio da linha de base. 


\subsection{Mapeamento da transferência de informação e o modelo estru- tural do circuito neural do sistema neuromotor}

Com base nos resultados para cada tipo de neurônio identificado no sistema neuromotor do inseto, montou-se um mapeamento dos tempos de atraso das máximas coerências da transferência de informação do circuitos neurais. Os resultados do mapeamento estrutural são apresentados nas Tabelas 5.6 e 5.7. A partir dos procedimentos de normalização das medidas, foi possível realizar uma análise conjunta de todos dos dados disponíveis, nas quais são mostrados os compartivos dos tempos de atrasos e da quantidade de informação transferida entres as conexões neurais do circuito neuromotor do inseto.

A partir dos resultados obtidos sobre as ocorrências dos picos de máxima coerência de informação e dos tempos de atraso, obteve-se a caracterização através da identificação de dois picos de atrasos para os interneurônios spiking, três picos para o interneurônios nonspiking e uma pequena variação de tempos para os neurônios sensores, pois esses últimos possuem um único padrão de caminho entre o apódema e o $\mathrm{FeCO}$.

Na Figura 5.20 é apresentado o resultado da proposta da estrutura de um circuito neural. O modelo de circuito neural foi gerado computacionalmente, a partir dos algoritmos desenvolvidos neste trabalho. Utilizou-se o software de visualização de grafos e objetos chamado GraphViz ${ }^{1}$. Desta forma, foram obtidos dos algoritmos a geração desses tipos de figuras com base nos dados obtidos dos experimentos.

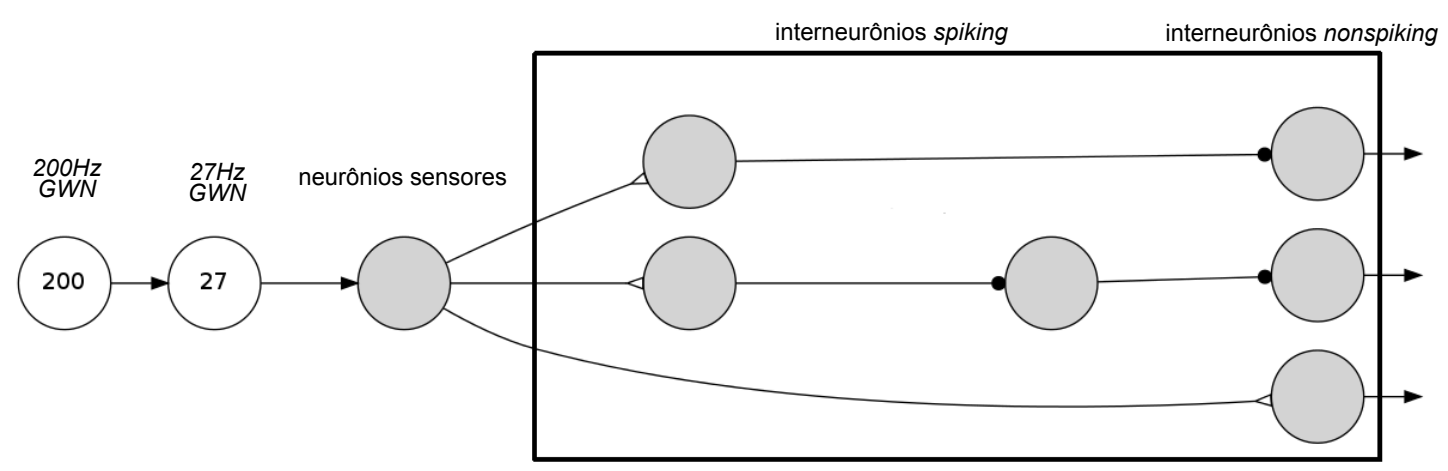

Figura 5.20: Esquemático do circuito neural do sistema neuromotor do gafanhoto. Um códigofonte foi desenvolvido para a obtenção desses tipos de circuitos neurais. Apresentam-se os geradores da excitação $(200 \mathrm{~Hz}$ e $27 \mathrm{~Hz})$, a região sensora neural (FeCO - Femoral Chordotonal Organ) e os interneurônios (spiking e nonspiking).

\footnotetext{
${ }^{1}$ Disponível em: www.graphviz.org
} 


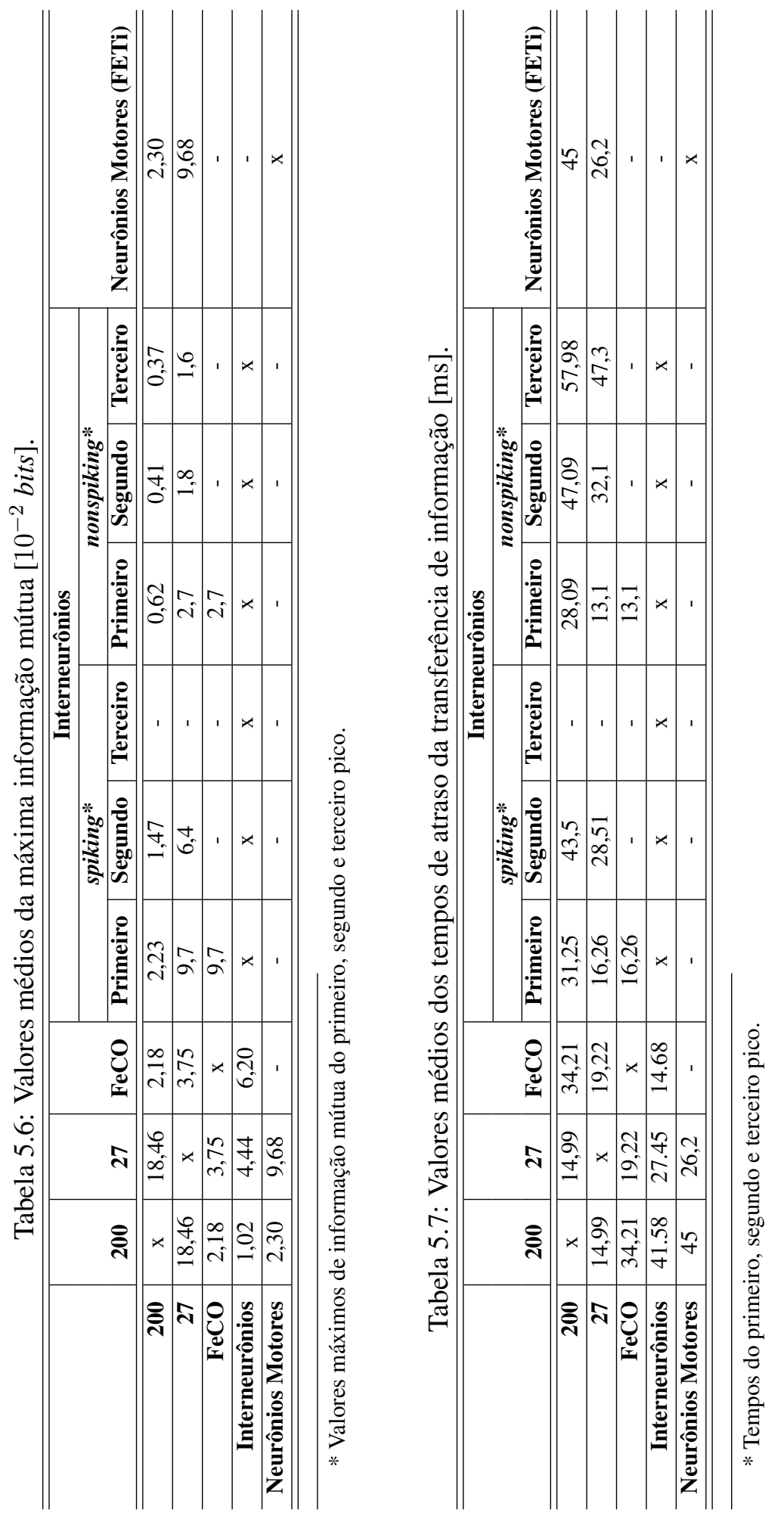


A partir dos dados apresentados nas Tabelas 5.6 e 5.7, foram mapeadas as conexões do circuito neural do sistema neuromotor do gafanhoto. A Figura 5.21 apresenta o principal resultado do diagrama de conexões neurais. Este grafo representa o circuito neural esperado entre o gânglio metatorácico e os neurônios motores do inseto.

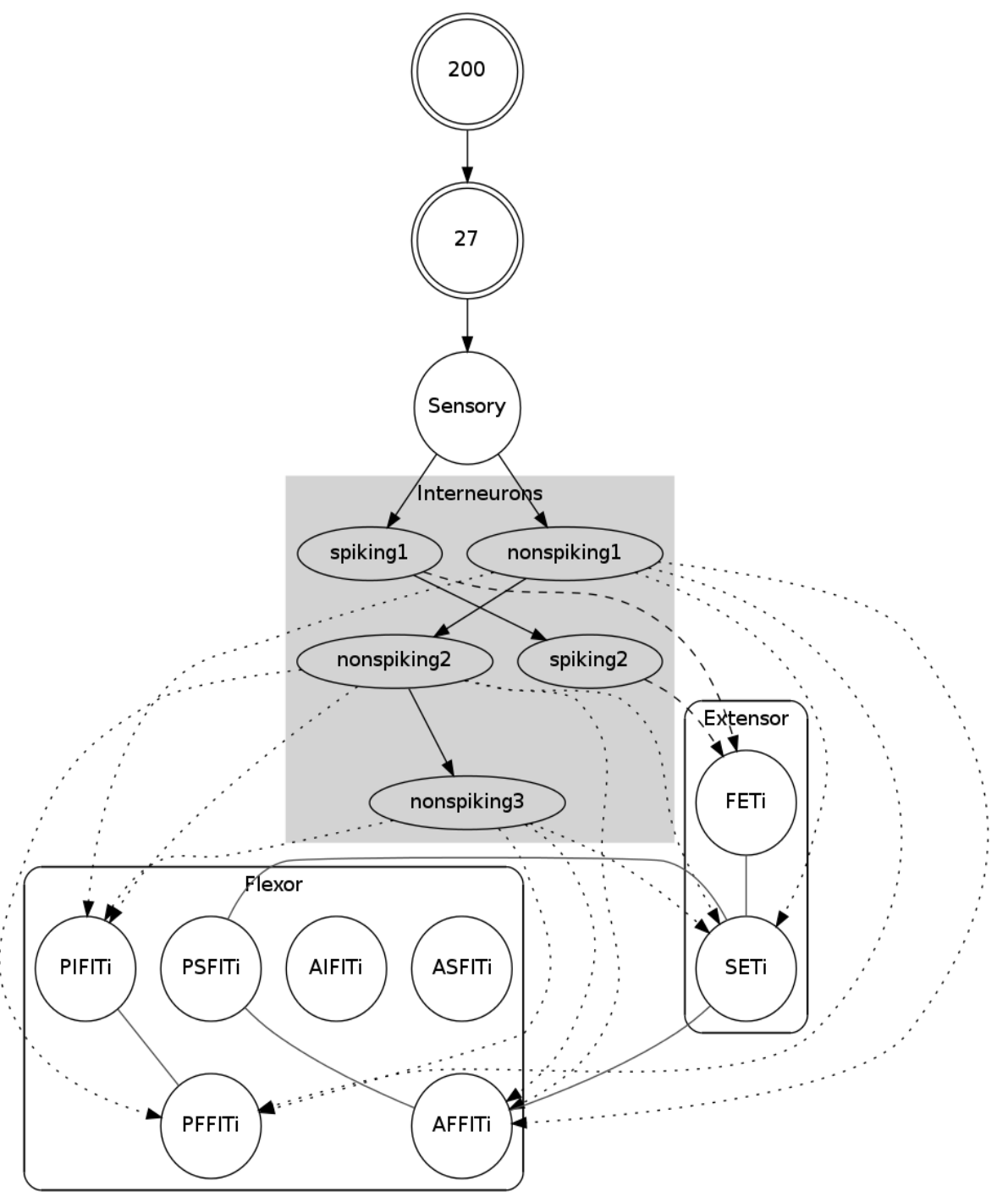

Figura 5.21: Grafo de conexões do gânglio metatorácico do sistema neuromotor do gafanhoto. Apresentam-se os geradores da excitação $(200 \mathrm{~Hz}$ e $27 \mathrm{~Hz})$, a região sensora neural $(\mathrm{FeCO})$, os interneurônios (spiking e nonspiking) e as estruturas dos neurônios motores.

Os principais caminhos no circuito neural foram identificados neste trabalho, dos quais destacam-se: os neurônios sensores do $\mathrm{FeCO}$, os interneurônios locais spiking e nonspiking e os neurônios motores. O neurônio com maior número de dados disponíveis para a análise é o FETi; no entanto, mesmo em menor quantidade de dados, os demais neurônios motores foram mapeados e considerados. São necessários novos ensaios e experimentos para a coleta de dados 
do inseto, pois algumas conexões possuem poucos dados para a análise das forças associação entre as estruturas neurais.

Para se fazer uma inferência relacionada às ações dos interneurônios sobre os neurônios motores, utilizou-se a correlação cruzada. Isso só é possível, pois as medidas de associação entre os neurônios possuem componentes lineares, conforme descrito em Dewhirst et al. (2009). A correlação cruzada revela a polaridade das conexões dos neurônios do circuito neural. Observou-se que o pico de correlação cruzada sobre o neurônio sensor é sempre positivo em todos os casos analisados. Nos interneurônios spiking, o primeiro pico é positivo e o segundo, negativo na maioria dos interneurônios. Nos interneurônios nonspiking, o primeiro pico é geralmente positivo; o segundo, negativo e o terceiro, positivo. A Figura 5.22 ilustra a ação dos interneurônios operando através dos EPSP ou IPSP. 

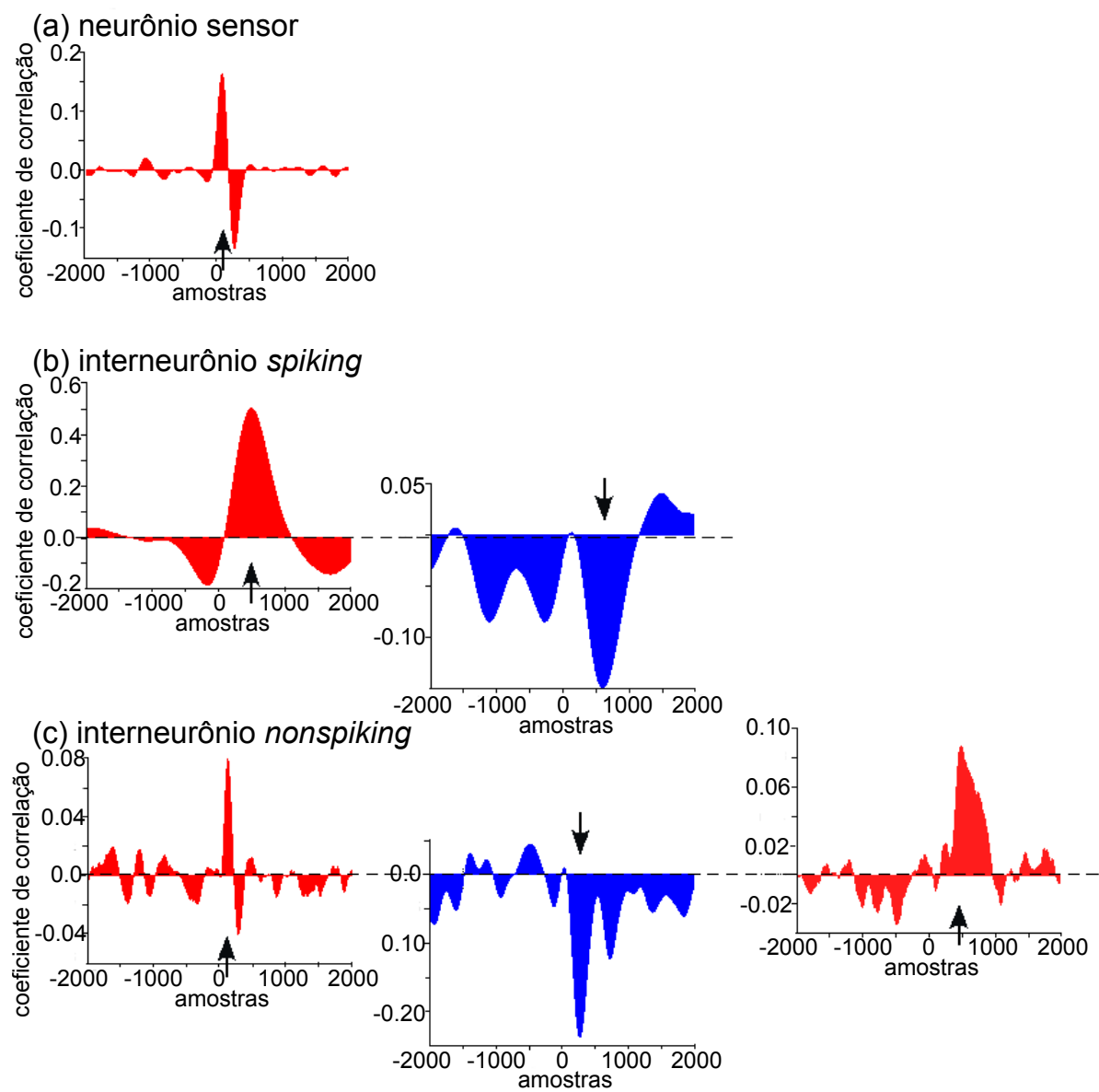

Pico 1

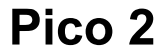

Pico 3

Figura 5.22: Polaridades da conectividade neural para inferências relacionadas à ação dos interneurônios nos neurônios motores, provando EPSP ou IPSP. A correlação cruzada revela a polaridade das conexões dos neurônios do circuito neural.(a) $\mathrm{O}$ pico de correlação cruzada sobre o neurônio sensor é sempre positivo em todos os 12 neurônios analisados. (b) Nos interneurônios spiking, o primeiro pico é positivo e o segundo é negativo na maioria dos interneurônios. (c) Nos interneurônios nonspiking, o primeiro pico é geralmente positivo; o segundo, negativo e o terceiro, positivo. As setas indicam os tempos de ocorrência dos picos obtidos da informação mútua atrasada. Os coeficientes de correlação variam entre -1 e +1 em que representam correlação máxima inversamente ou diretamente proporcional, respectivamente. 


\section{Capítulo 6}

\section{Publicações e Submissões}

Publicações relacionadas ao trabalho, em periódico:

- Aplicação de um modelo de inferência bayesiana para tempos de atraso: Endo, W., Maciel, C. D., Scalassara, P. R. e Hirono, F. C. Direction of arrival uncertaninty of adaptive beamformer based on a posteriori information (2014). In Journal of Control, Automation and Electrical Systems, DOI: 10.1007/s40313-013-0092-4, Springer US.

Em congresso internacional:

- Análise de sensiblidade utilizando informações mútuas condicionadas de ordens superiores: Endo, W., Maciel, C. D., Simpson, D., e Newland, P. L. (2013). Multiple pathways network description of motor neurons in locusts: An information sensitivity analysis. In $4^{o}$ Biosignals and Biorobotics Conference (BRC), 2013 ISSNIP, pp. 1-6. (Disponível no IEEE Xplorer $^{1}$ )

Submissões relacionadas ao trabalho, em periódicos:

- Inferência dos padrões de conexões sinápticas pela informação mútua atrasada: Endo, W., Maciel, C. D., Simpson, D., e Newland, P. L. Delayed Mutual Information Infers Patterns of Synaptic Connectivity in a Proprioceptive Neural Network. (2014) PLoS Computational Neuroscience.

\footnotetext{
${ }^{1}$ Site de acesso: http://ieeexplore.ieee.org/
} 
Outros artigos aceitos/publicados relacionados ao trabalho de pesquisa:

- Resultados iniciais da análise de sensibilidade (artigo aceito em congresso internacional): Endo, W., Maciel, C. D., Simpson, D., e Newland, P. L. Description Network with Multiple Pathways from Sensory Neurons in Locusts. (2012) In $4^{o}$ International Conference on Neural Computation Theory and Applications (NCTA), IJCCI 2012.

- Aplicação de um modelo de inferência bayesiana para tempos de atraso (versão resumida): Endo, W., Maciel, C. D. e Scalassara, P. R. Direction of arrival uncertaninty of adaptive beamformer based on a posteriori information (2011). In X Conferência Brasileira de Dinâmica, Controle e Aplicações, DINCON 2011.

- Aplicação de um modelo dinâmico com atrasos de tempo: Endo, W., Maciel, C. D., Scalassara, P. R., Bózoli L. A., Projeto, simulação e caracterização de um transdutor de arranjo anular com focalização dinâmica. (2010) In XVIII Congresso Brasileiro de Automática (CBA), CBA 2010.

- Identificação de sistemas de resposta impulsiva baseada em modelo: Musolino, B. C., Maciel C. D., Pereira, A. H. A., Rodrigues, J. A., Endo, W. Algoritmo para determinação do coeficiente de amortecimento de materiais pela técnica da excitação por impulso. (2010) In XVIII Congresso Brasileiro de Automática (CBA), CBA 2010. 


\section{Capítulo 7}

\section{Considerações finais e próximas etapas}

Neste capítulo apresentam-se as considerações finais sobre as contribuições mais relevantes desta pesquisa. A qual envolve, desde as aplicações em modelos bioinspirados, até a metodologia de estimativa de medidas de associação e transferência de informação para os sistemas neurais estudados. Além disso, comentam-se algumas novas hipóteses para descrever fisiologicamente o sistema neuromotor, que surgiram através dos resultados desta pesquisa. Em seguida, discutem-se as principais contribuições científicas específicas para os circuitos neuromotores dos gafanhotos. Por fim, apresentam-se as considerações finais e o apontamento dos próximos passos e possibilidades de linhas de pesquisa.

\section{Considerações sobre os métodos e resultados}

Primeiramente, foi apresentado um modelo de associação de primeira ordem, o STA. Esse modelo tem em comum as propriedades do primeiro termo no kernel das respostas dos modelos de Volterra ou Wiener. Os resultados obtidos a partir desse modelo seguiu esta linha de pesquisa, como apresentado anteriormente em Kondoh et al. (1995), Newland e Kondoh (1997a), Newland e Kondoh (1997b) e Vidal-Gadea et al. (2010). Assim, a análise de STA foi o ponto de partida para o início dos novos estudos.

Verificou-se que as respostas dos STA, com as respostas de correlação cruzada possuem características similares. Isso ocorre, pois as duas abordagens dependem de valores médios e estatísticos de primeira ordem. Após gerarem-se os resultados com o STA, isso permitiu que se 
realizasse uma inferência bayesiana com o objetivo de se apresentar um modelo inicial de previsibilidade, baseado nas dependências condicionais das $p d f$ 's de estímulo e ocorrência de spikes. O modelo utiliza como informação a priori, $p($ spike $)$, quando é conhecida a curva de estímulo, $p($ stim $)$. E, juntamente com a probabilidade condicional de verosimilhança, $p$ (stim|spike), obtém-se pela inferência bayesiana a informação a posteriori, $p($ spike|stim). Essa análise permite que se associe o estímulo do neurônio sensor à geração de spikes no $\mathrm{FeCO}$, sendo assim uma importante contribuição da análise de STA.

Em seguida, foi apresentada uma metodologia para estimar o nível de transferência de informação entre sistemas neurais, com amostras de tamanhos variados e diferentes forças de associação da informação. Foram gerados dados sintéticos para os testes computacionais dessas ferramentas. A partir desses dados simulados, foi possível analisar diversos parâmetros dos algoritmos propostos da $D M I$ e da $T E$. Como era esperado, os tamanhos variados das amostras geram erros de desvio sistemático que devem ser compensados e normalizados para a análise conjunta dos dados.

Para os testes de comparação e validação, foram utilizados os Surrogate Data como ferramenta de análise de significância estatística dos resultados. Essa técnica determina a compensação do nível de erro de desvio, quando são analisadas as informações das linhas de base nas curvas de atraso da transferência da informação. Para isso, estabeleceram-se limites para os níveis de significância estatísticas dos resultados de Surrogate Data. Definindo-se, assim, os ajustes iniciais dos algoritmos para os dados sintéticos, que em seguida puderam ser aplicados aos dados reais.

Ao se aplicar os algoritmos nos sinais do bancos de dados dos experimentos com gafanhotos, obtiveram-se relevantes resultados. Pois as ferramentas de teoria de informação mostraram-se efetivas para o cálculo do atraso de máxima coerência da informação. A partir disso, a $D M I$ ou a $T E$ apresentaram novas discussões sobre as vantagens ou desvantagens de aplicação de cada ferramenta. Observou-se que a $D M I$ apresenta uma melhor qualidade dos resultados e consegue identificar melhor os atrasos de transferência de informação. Enquanto que a $T E$ consegue identificar melhor as características de direção do fluxo de informação. Isso acontece, devido às diferentes características da dimensionalidade estatística de cada ferramenta. Pois, enquanto que a $D M I$ depende de um espaço em $\mathrm{A}^{2}$ (espaço bidimensional), pela sua dependência apenas de $X$ e $Y$; a $T E$ depende das probabilidades conjuntas condicionais, $X$ e $Y^{\tau}$, dado $Y_{k}^{\tau}$ e ocupa um espaço em $\mathrm{A}^{3}$ (espaço tridimensional). Assim, deve-se entender que 
o problema do tamanho das amostras pode gerar erros de desvios, devido à dimensionalidade estatística de cada ferramenta.

O banco de dados, com os sinais neurais, possui um grande número de experimentos com o sistema proprioceptor de gafanhotos do deserto. Uma das limitações do banco de dados é a inexistência de coletas simultâneas entre diferentes partes do mesmo inseto. Assim, quando se desejou medir e comparar medidas de diferentes experimentos, foi necessário adotar ferramentas que compensassem e padronizassem as medidas dos dados coletados. Para isso, foram utilizados os algoritmos, já testados com dados sintéticos, das medidas de normalização. Essa medida utiliza, como fator de normalização, a análise das entropias totais do sistema. Para a $D M I$, a normalização foi baseada nas entropias de $X$ e $Y$, enquanto que, para a $T E$, as medidas de normalização utilizaram as propriedades da $C M I$ para quantificar a entropia total do sistema.

Com base nas medidas de normalização e compensação dos erros de desvio, a metodologia proposta estimou o nível de transferência de informação para os casos nos quais existem problemas de ruído na coleta dos dados ou erros de medida de informação, ocasionada pelas amostras de diferentes tamanhos. Mostrou-se que o nível de transferência de informação, $\psi_{\Delta}$, é uma medida padronizada para a análise conjunta dos dados.

Ao se analisar de forma conjunta os dados, definiram-se padrões de diferentes atrasos e valores de máxima coerência da informação. Foram identificados três padrões de comportamento. O primeiro, para os neurônios sensores, com um limite de variação de tempos bem definidos, de fato confirmado, pois anatomicamente sabe-se da existência de apenas um caminho entre o apódema e os neurônios sensores do $\mathrm{FeCO}$. O segundo, para os interneurônios spiking, teve identificado 2 padrões de caminho no gânglio metatorácico do inseto, e o terceiro padrão teve um total de 3 diferentes caminhos identificados. Ao se mapear esses múltiplos caminhos para diferentes tipos de neurônios, obteve-se um mapeamento geral do circuito neural que controla as pernas traseiras do gafanhoto. Foi aplicado um classificador de grupos baseado no $B I C$ de suas misturas de gaussianas para todo o conjunto de dados. O classificador indicou padrões de múltiplos caminhos, que condizem com o mesmo modelo anatômico e funcional, conforme é descrito na literatura por Burrows (1996). A partir dessas evidências, obteve-se um mapeamento geral do circuito neural de controle local do membro inferior do gafanhoto.

Para os resultados com os interneurônios locais, em que foi identificado mais de um padrão de caminho, a TE torna-se necessária para expressar melhor a informação e diferentes tempos de atrasos. Os múltiplos padrões de caminhos geram níveis de associação identificados 
indiretamente através do uso da $T E$.

Desta forma, uma das principais contribuições deste trabalho foi apresentar um modelo geral do sistema proprioceptor de gafanhotos, baseado nas técnicas propostas de normalização e compensação dos erros de desvio ocorridos entre os diferentes experimentos.

\section{Novas interpretações sobre os sinais neurais na propriocepção do inseto}

Após a análise do $S T A$ e da $D M I$ para os neurônios do $F e C O$, observaram-se as pequenas variações dos tempos de atraso. Em um primeiro instante, essas diferenças de valores não mostraram ter algum significado, pois os valores médios desses tempos condiziam com um único padrão de caminho. No entanto, ao se comparar o $\mathrm{FeCO}$ com fuso muscular nos mamíferos, conforme Fouad et al. (2003), algumas questões puderam ser levantadas.

Foram identificados dois padrões de comportamento do $\mathrm{FeCO}$, e isso foi observado tanto nos resultados do STA quanto da DMI. Ao se analisarem todos os resultados dos neurônios sensores entre si, os resultados obtidos apontaram para algumas evidências de comportamentos distintos dentro da própria estrutura do $\mathrm{FeCO}$.

Tomando-se a analogia de comportamento entre o $\mathrm{FeCO}$ e o fuso muscular, a nova hipótese tomou forma. E observou-se que as variações de tempo ilustram comportamentos funcionais distintos que podem se referir à sensibilidade de movimento e à aplicação de força gerada na excitação do apódema do FeCO (Fouad et al., 2003) (Dewhirst et al., 2013). No entanto, essa hipótese deve ser analisada com maior profundidade, a fim de se obterem novas evidências que descrevam, de forma satisfatória, o comportamento desses neurônios sensores.

\section{Perspectivas de trabalhos futuros e próximos passos}

As próximas etapas deste trabalho estão focadas na obtenção de mais resultados para efeito comparativo e para testes com outras abordagens do sistema neuromotor. A partir das novas hipóteses e objetivando aprimorar o modelo de circuitos neurais do inseto, novos experimentos neurofisiológicos são necessários.

Com relação à parte computacional, algumas novas abordagens podem ser viáveis para se analisar as medidas de associação. Uma interessante análise para as séries temporais biológicas seria verificar as dependências condicionais sujeitas aos atrasos, $\tau$, entre diferentes pontos do circuito neuromotor. No entanto, isso depende de novos experimentos. Mas essa abordagem 
poderia enriquecer as informações sobre a conectividade entre diferentes partes do sistema neuromotor. Na Figura 7.1 é mostrado esse caso; trata-se de um sistema com 3 vértices. Cada um é definido por, $X, Y$ e $Z$, representando diferentes pontos do sistema neuromotor.

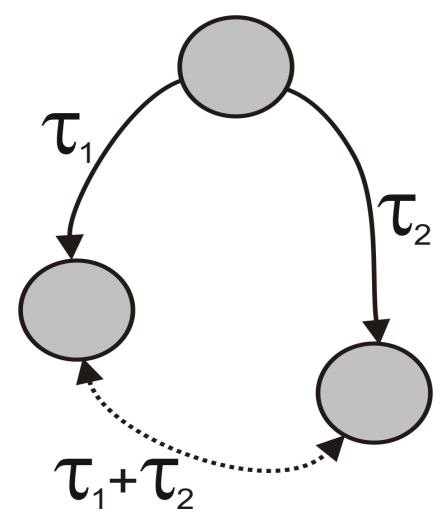

Figura 7.1: Grafo para informação mútua condicionada. A este modelo podem ser associados diferentes tempos de atraso, $\tau_{1}$ e $\tau_{2}$, como na DMI. Tendo como principal diferença a existência de 3 sinais distintos, $X, Y$ e $Z$.

A $T E$, computacionalmente, está baseada em uma ideia similar de dimensionalidade. Nessa abordagem, o valor de $X$ é o um histórico comum entre os sinais $Y$ e $Z$. Pois o sinal $X$ introduz a característica dinâmica da $T E$. No entanto, ao se utilizar séries temporais distintas, é possível aplicar o conhecimento desta terceira série temporal, $Z$, na informação mútua condicional, $I(X ; Y \mid Z)$, com sobreposição dos diferentes tempos de atraso. Isso permitiria também aplicar um novo modelo da $T E$ para mais de 2 séries temporais.

A etapa inicial desse tipo de análise pode ser feita para os neurônios sensores e motores utilizando-se a análise de sensibilidade da informação. A informação de análise de sensibilidade (Critchfield et al., 1986) estima a influência de diferentes entradas (neurônios sensores) através de um neurônio motor. Esse modelo pode ser aplicado a ordens superiores de informação mútua condicionada, dadas por $I\left(X_{1}, X_{2}, \ldots, X_{n} \mid Y\right)$ (Lüdtke et al., 2008). Os valores de $X_{1}, X_{2}, \ldots, X_{n}$ podem ser associados aos neurônios sensores e ao efeito de suas respectivas sensibilidades para $Y$. Na Figura 7.2, é apresentado um modelo de interações em um sistema, utilizando como conexões as informações mútuas condicionadas entre os diferentes sinais.

A partir das interações entre os pares, podem ser observadas suas influências individuais na saída deste neurônio. Essa técnica, também da teoria de informação, é viável para a estimativa da interações do emparelhamento entre entradas e a saída de um sistema neural (Endo et al., 2013b). Além disso, a análise de sensibilidade permite utilizar sinais de entradas dependentes ou com algum nível de correlação, ao contrário da análise de variância (ANOVA), que permite 


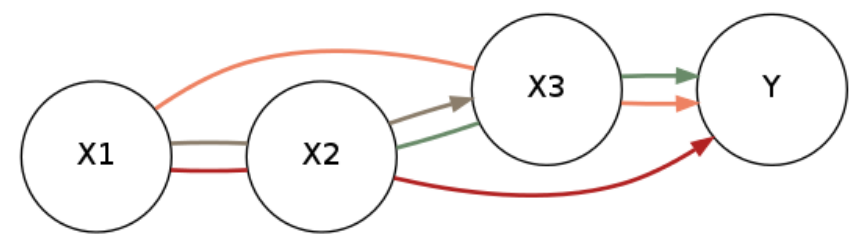

Figura 7.2: Representação de um sistema de informação mútua condicionada. Cada borda colorida representa um possível emparelhamento de sinais.

somente entradas não correlacionadas. 


\section{Referências Bibliográficas}

Alonso, J. F., Mananas, M. A., Topor, D. H. Z. L., e Bruce, E. N. (2007). Evaluation of respiratory muscles activity by means of cross mutual information function at different levels of ventilatory effort. IEEE Treans. on Biomedical Eng., 54(9):1573-1582.

Ang, K. K., Chin, Z. Y., Zhang, H., e Guan, C. (2012). Mutual information-based selection of optimal spatial and temporal patterns for single-trial eeg-based $\{\mathrm{BCIs}\}$. Pattern Recognition, 45(6):2137 - 2144. Brain Decoding.

Besson, P., Richiardi, J., Bourdin, C., Bringoux, L., Mestre, D., e Vercher, J.-L. (2010). Bayesian networks and information theory for audio-visual perception modeling. Biological $\mathrm{Cy}$ bernetics, 103:213-226. 10.1007/s00422-010-0392-8.

Bettencourt, L. M. A., Gintautas, V., e Ham, M. I. (2008). Identification of functional information subgraphs in complex networks. Phys. Rev. Lett., 100:238701.

Bjornsdotter, M. e Wessberg, J. (2010). Identification and localization of human brain activity patterns using particle swarm optimization. In Computer and Automation Engineering (ICCAE), 2010 The 2nd International Conference on, vol. 5, pp. 632-636.

Burrows, M. (1987). Parallel processing of proprioceptive signals by spiking local interneurons and motor neurons in the locust. Journal of Neuroscience, 7:1064-1080.

Burrows, M. (1996). The Neurobiology of an Insect Brain. Oxford University Press, USA.

Burrows, M., Laurent, G., e Field, L. H. (1988). Proprioceptive inputs to nonspiking local interneurons contribute to local reflexes of a locust hindleg. Journal of Neuroscience, 8:30853093.

Byrne, J. H. (1997). Neuroscience online: An electronic textbook for the neurosciences. http://nba.uth.tmc.edu/neuroscience/. [Acessado em 20-Abril-2013]. 
Cover, T. M. e Thomas, J. A. (2006). Elements of Information Theory. Wiley-Interscience, 2 ed.

Critchfield, G. C., Willard, K. E., e Connelly, D. P. (1986). Probabilistic sensitivity analysis methods for general decision models. Computers and Biomedical Research, 19(3):254-265.

Darbellay, G. A. e Wuertz, D. (2000). The entropy as a tool for analysing statistical dependences in financial time series. Physica A Statistical Mechanics and its Applications, 287:429439.

Dayan, P. e Abbott, L. F. (2001). Theoretical Neuroscience: computational and mathematical modeling of neural systems. Massachusetts Institute of Technology, 1 ed.

Devasahayam, S. R. (2013). Signals and Systems in Biomedical Engineering - Signal Processing and Physiological Systems Modeling. Springer, 2 ed.

Dewhirst, O. P., Angarita-Jaimes, N., Simpson, D. M., Allen, R., e Newland, P. L. (2013). A system identification analysis of neural adaptation dynamics and nonlinear responses in the local reflex control of locust hind limbs. Journal of Computational Neuroscience, 34(1):3958.

Dewhirst, O. P., Simpson, D. M., Allen, R., e Newland, P. L. (2009). Neuromuscular reflex control of limb movement - validating models of the locusts hind leg control system using physiological input signals. In Proceedings of the 4th International IEEE EMBS Conference on Neural Engineering.

DiCaprio, R. (2004). Information transfer rate of nonspiking afferent neurons in the crab. Journal of neurophysiology, 92(1):302-310.

Diniz, P. S. R., da Silva, E. A. B., e Netto, S. L. (2004). Processamento Digital de Sinais Projeto e Análise de Sistemas. Editora Bookman.

Diniz, P. S. R., Silva, E. A. B., e Netto, S. L. (2002). Digital Signal Processing: System Analysis and Design. Cambridge University Press, Cambridge.

Dionisio, A., Menezes, R., e Mendes, D. A. (2004). Mutual information: a measure of dependency for nonlinear time series. Physica A, 344:326-329.

Dolan, K. T. e Spano, M. L. (2001). Surrogate for nonlinear time series analysis. Phys. Rev. E, 64:046128. 
Endo, W., Maciel, C., Scalassara, P., e Hirono, F. (2013a). Direction of arrival uncertainty of adaptive beamformer based on a posteriori information. Journal of Control, Automation and Electrical Systems, pp. 1-9.

Endo, W., Maciel, C., Simpson, D., e Newland, P. (2013b). Multiple pathways network description of motor neurons in locusts: An information sensitivity analysis. In Biosignals and Biorobotics Conference (BRC), 2013 ISSNIP, pp. 1-6.

Faes, L., Erla, S., e Nollo, G. (2012). Measuring connectivity in linear multivariate processes: Definitions, interpretation, and practical analysis. Computational and Mathematical Methods in Medicine, 2012:18.

Fouad, K., Fischer, H., e Büschges, A. (2003). Comparative psychology of motor systems. In Gallagher, M. e Nelson, R. J. (Eds.), Handbook of Psychology - Volume 3 - Biological Psychology, chapter 5, pp. 109 - 137. John Wiley and Sons, Inc.

Goldsmith, A. e Varaiya, P. (1996). Capacity, mutual information, and coding for finite-state markov channels. Information Theory, IEEE Transactions on, 42(3):868-886.

Gourévitch, B. e Eggermont, J. J. (March 2007). Evaluating information transfer between auditory cortical neurons. Journal of Neurophysiology, 97(3):2533-2543.

Greenblatt, R., Pflieger, M., e Ossadtchi, A. (2012). Connectivity measures applied to human brain electrophysiological data. Journal of Neuroscience Methods, 207(1):1 - 16.

Ince, R. A., Mazzoni, A., Bartels, A., Logothetis, N. K., e Panzeri, S. (2012). A novel test to determine the significance of neural selectivity to single and multiple potentially correlated stimulus features. Journal of Neuroscience Methods, 210(1):49 - 65 .

Ito, S., Hansen, M. E., Heiland, R., Lumsdaine, A., Litke, A. M., e Beggs, J. M. (2011). Extending transfer entropy improves identification of effective connectivity in a spiking cortical network model. PLoS ONE, 6(11):e27431.

Jiménez-Fabián, R. e Verlinden, O. (2012). Review of control algorithms for robotic ankle systems in lower-limb orthoses, prostheses, and exoskeletons. Medical Engineering and Physics, 34(4):397 - 408.

Jin, S.-H., Lin, P., e Hallett, M. (2010). Linear and nonlinear information flow based on timedelayed mutual information method and its application to corticomuscular interaction. Clinical Neurophysiology, 121(3):392 - 401. 
Kaiser, A. e Schreiber, T. (2002). Information transfer in continuous processes. Physica D: Nonlinear Phenomena, 166(1 - 2):43 - 62.

Kondoh, Y., Okuma, J., e Newland, P. L. (1995). Dynamics of neurons controlling movements of a locust hind leg: Wiener kernel analysis of the responses of proprioceptive afferents. Journal of Neurophysiology, 73(5):1829-1842.

Kugiumtzis, D. (2002). Surrogate data test on time series. In Soofi, A. e Cao, L. (Eds.), Modelling and Forecasting Financial Data, Techniques of Nonlinear Dynamics, chapter 12, pp. 267 - 282. Kluwer Academic Publishers.

Lee, J., Nemati, S., Silva, I., Edwards, B., Butler, J., e Malhotra, A. (2012). Transfer entropy estimation and directional coupling change detection in biomedical time series. BioMedical Engineering OnLine, 11(1):19.

Leuthardt, E. C., Schalk, G., Moran, D., e Ojemann, J. G. (2006). The emerging world of motor neuroprosthetics: A neurosurgical perspective. J. Neurosurgery, 59(1):1-14.

Lindner, M., Vicente, R., Viola, P., e Wibral, M. (2011). Trentool: A matlab open source toolbox to analyse information flow in time series data with transfer entropy. BMC Neuroscience, 12.

Liu, Y. e Aviyente, S. (2012). Quantification of effective connectivity in the brain using a measure of directed information. Computational and Mathematical Methods in Medicine, 2012.

Lüdtke, N., Panzeri, S., Brown, M., Broomhead, D. S., Knowles, J., Montemurro, M. A., e Kell, D. B. (2008). Information-theoretic sensitivity analysis: a general method for credit assignment in complex networks. Journal of the Royal Society Interface, 5:223-235.

Maciel, C. D., Simpson, D. M., Allen, R., e Newland, P. L. (2012). Inference about multiple pathways in motor control limb in locust. In Proceedings of the Biosignal.

Manolakis, D. G., Ingle, V. K., e Kogon, S. M. (2005). Statistical and Adaptive Signal Processing - Spectral Estimation, Signal Modeling, Adaptive Filtering and Array Processing. Artech House.

Marschinski, R. e Kantz, H. (2002). Analysing the information flow between financial time series. The European Physical Journal B - Condensed Matter and Complex Systems, 30:275281. 10.1140/epjb/e2002-00379-2. 
McDonnell, M., Ikeda, S., e Manton, J. (2011). An introductory review of information theory in the context of computational neuroscience. Biological Cybernetics, 105:55-70.

Meisel, M., Pappas, V., e Zhang, L. (2010). A taxonomy of biologically inspired research in computer networking. Computer Networks, 54(6):901 - 916. New Network Paradigms.

Newland, P. L. e Kondoh, Y. (1997a). Dynamics of neurons controlling movements of a locust hind leg ii. flexor tibiae motor neurons. Journal of Neurophysiology, 77(4):1731-1746.

Newland, P. L. e Kondoh, Y. (1997b). Dynamics of neurons controlling movements of a locust hind leg iii. extensor tibiae motor neurons. Journal of Neurophysiology, 77(6):3297-3310.

Nichols, J. (2006). Examining structural dynamics using information flow. Probabilistic Engineering Mechanics, 21(4):420 - 433.

Nichols, J. M., Seaver, M., Trickey, S. T., Todd, M. D., Olson, C., e Overbey, L. (2005). Detecting nonlinearity in structural systems using the transfer entropy. Phys. Rev. E, 72:046217.

Nicácio, L. R., Maciel, C., Simpson, D., Newland, P., e T., N. G. (2012). Identification of the stationarity in biological time series. In Anais do XIX Congresso Brasileiro de Automática (CBA), pp. 3482-3489.

Park, I. M. M. e Pillow, J. W. (2011). Bayesian spike-triggered covariance analysis. In ShaweTaylor, J., Zemel, R., Bartlett, P., Pereira, F., e Weinberger, K. (Eds.), Advances in Neural Information Processing Systems 24, pp. 1692-1700. Neural Information Processing Systems Foundation.

Pflüger, H.-J. e Duch, C. (2011). Dynamic neural control of insect muscle metabolism related to motor behavior. Physiology, 26(4):293-303.

Pillow, J. W. e Simoncelli, E. P. (2006). Dimensionality reduction in neural models: An information-theoretic generalization of spike-triggered average and covariance analysis. Journal of Vision, 6(4):414-428.

Pätschke, A., Bicker, G., e Stern, M. (2004). Axonal regeneration of proctolinergic neurons in the central nervous system of the locust. Developmental Brain Research, 150(1):73 - 76.

Romero, S. M. B. (2000). Fundamentos de Neurofisiologia comparada - da recepção à integração. Holos Editora - Fapesp, 1 ed. 
Runge, J., Heitzig, J., Petoukhov, V., e Kurths, J. (2012). Escaping the curse of dimensionality in estimating multivariate transfer entropy. Phys. Rev. Lett., 108:258701.

Schreiber, T. (2000). Measuring information transfer. Phys. Rev. Lett., 85:461-464.

Schreiber, T. e Schmitz, A. (1996). Improved surrogate data for nonlinearity tests. Phys. Rev. Lett., 77:635.

Schreiber, T. e Schmitz, A. (2000). Surrogate time series. Phys. D, 142(3-4):346-382.

Schwartz, O., Pillow, J. W., Rust, N. C., e Simoncelli, E. P. (2006). Spike-triggered neural characterization. Journal of Vision, 6(4):484-507.

Shannon, C. E. (1948). A mathematical theory of communication. Bell System Technical Journal, 27(3):379 - 423.

Shimazaki, H. e Shinomoto, S. (2007). A method for selecting the bin size of a time histogram. Neural Computation, MIT Press, 19(6):1503 - 1527.

Silchenko, A. N., Adamchic, I., Pawelczyk, N., Hauptmann, C., Maarouf, M., Sturm, V., e Tass, P. A. (2010). Data-driven approach to the estimation of connectivity and time delays in the coupling of interacting neuronal subsystems. Journal of Neuroscience Methods, 191(1):32 -44 .

Sporns, O. (2011). Networks of the Brain. The MIT Press, 1 ed.

Strehl, A. e Ghosh, J. (2003). Cluster ensembles — a knowledge reuse framework for combining multiple partitions. J. Mach. Learn. Res., 3:583-617.

van Steen, M. (2010). Graph Theory and Complex Networks. Marteen van Steen, 1 ed.

Vaseghi, S. V. (2006). Advanced Digital Signal Processing and Noise Reduction. John Wiley Ltd.

Venema, V., Ament, F., e Simmer, C. (2006). A stochastic iterative amplitude adjusted fourier transform algorithm with improved accuracy. Nonlinear Processes in Geophysics, 13(3):321-328.

Vicente, R., Wibral, M., Lindner, M., e Pipa, G. (2011). Transfer entropy - a model-free measure of effective connectivity for the neurosciences. Journal of Computational Neuroscience, 30(1):45-67. 
Vidal-Gadea, A., Jing, X. J., Simpson, D. M., Dewhirst, O., Kondoh, Y., e Newland, R. A. P. (2010). Coding characteristics of spiking local interneurons during imposed limb movements in the locust. Journal of Neurophysiology, 103:603-615.

Wilmer, A., de Lussanet, M., e Lappe, M. (2012). Time-delayed mutual information of the phase as a measure of functional connectivity. PLOS ONE, 7(9):e44633.

Wilson, R. J. (1996). Introduction to Graph Theory. Prentice Hall, 4 ed.

Yang, C., Jeannès, R. L. B., Faucon, G., e Shu, H. (2013). Detecting information flow direction in multivariate linear and nonlinear models. Signal Processing, 93(1):304 - 312.

Ying, C., Jia-fan, Z., Can-jun, Y., e Bin, N. (2007). Design and hybrid control of the pneumatic force-feedback systems for arm-exoskeleton by using on/off valve. Mechatronics, 17(6):325 -335 .

Zak-Szatkowska, M. e Bogdan, M. (2011). Modified versions of the bayesian information criterion for sparse generalized linear models. Computational Statistics and Data Analysis, 55(11):2908 - 2924.

Zheng, Y.-J., Chen, S.-Y., Lin, Y., e Wang, W.-L. (2013). Bio-inspired optimization of sustainable energy systems: A review. Mathematical Problems in Engineering, 2013(Article ID 354523):12p. 


\section{ANEXO A:}

\section{Descrição dos lotes para a análise}

Neste apêndice, apresentam-se os grupos analisados para cada tipo de neurônio. Eles foram divididos em lotes. São definidos 3 lotes principais: dos neurônios do $\mathrm{FeCO}$, dos interneurônios locais spiking e dos interneurônios locais nonspiking. Cada lote é um arquivo em que é carregado pelos algoritmos para a análise conjunta dos dados. A primeira coluna representa o nome do arquivo (ID-922.txt.gz). A segunda e a terceira colunas representam os canais dos experimentos que são escolhidos para análise, definidos nesta ordem, $(2 \rightarrow 0$, por exemplo). A penúltima e a última coluna representam o início e o fim, respectivamente, do trecho selecionado para análise. Os valores são definidos por amostras que devem ser calculadas em função do tempo, utilizando-se a respectiva frequência de amostragem, $F_{s}$.

Lote 1 - Neurônios sensores do FeCO:

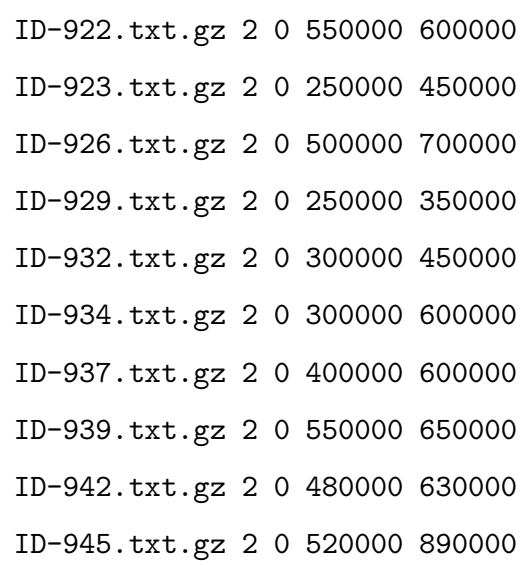

Lote 2 - Interneurônios locais spiking:

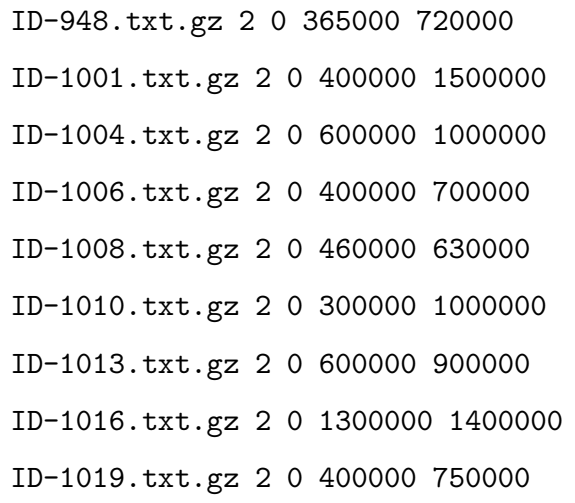

ID-505.txt.gz 21380000480000

ID-506.txt.gz 21315000480000 
ID-508.txt.gz 21380000580000 ID-515.txt.gz 21600000840000 ID-518.txt.gz 21720000800000 ID-519.txt.gz 21580000680000 ID-523.txt.gz 21760000940000 ID-538.txt.gz 21480000720000 ID-539.txt.gz 2111000001300000 ID-543.txt.gz 21820000940000 ID-545.txt.gz 21620000780000 ID-549.txt.gz 21360000600000 ID-602.txt.gz 21530000660000 ID-607.txt.gz 21480000720000

Lote 3 - Interneurônios locais nonspiking:

ID-919.txt.gz 30340000580000 ID-1038.txt.gz 30330000510000 ID-1211.txt.gz 20350000750000 ID-1304.txt.gz 20330000480000 ID-1321.txt.gz 20360000480000 ID-1405.txt.gz 30550000700000 ID-1410.txt.gz 30800000900000 ID-1413.txt.gz 30350000550000 ID-1419.txt.gz 30480000540000 ID-1422.txt.gz 30500000720000 ID-1424.txt.gz 30500000720000 ID-1431.txt.gz 30450000550000 ID-1434.txt.gz 30450000550000
ID-609.txt.gz 21650000780000 ID-612.txt.gz 21600000750000 ID-620.txt.gz 21550000700000 ID-621.txt.gz 21600000750000 ID-622.txt.gz 21660000860000 ID-626.txt.gz 21560000760000 ID-634.txt.gz 21550000650000 ID-641.txt.gz 21630000780000 ID-647.txt.gz 21600000750000 ID-722.txt.gz 21520000680000

ID-1435.txt.gz 30450000580000 ID-1436.txt.gz 30450000580000 ID-1441.txt.gz 30250000450000 ID-1442.txt.gz 20190000230000 ID-1443.txt.gz 20250000420000 ID-1501.txt.gz 30625000680000 ID-1504.txt.gz 30520000620000 ID-1505.txt.gz 30520000620000 ID-1507.txt.gz 30400000510000 ID-1508.txt.gz 30540000620000 ID-1512.txt.gz 30530000620000 ID-1516.txt.gz 30780000950000 


\section{ANEXO B:}

\section{Descrição dos arquivos do banco de dados}

Neste apêndice, apresenta-se o arquivo de descrição de todos os experimentos, disponíveis no banco de dados dos sinais neurais do inseto. Este arquivo é utilizado pelos algoritmos para se obter as informações de coleta dos dados. A primeira coluna aponta para o endereço do experimento ( $I D-501$, por exemplo). A segunda coluna informa a largura de banda da frequência do estímulo mecânico do fórceps que excita o apódema. A terceira é a frequência de amostragem, $F_{s}$, com a qual o experimento foi coletado. Da quarta à sétima coluna são mostrados os nomes dos canais gravados de cada experimento. O nome $X$ se refere a uma localização não identificada no momento da coleta de dados. As siglas FETI, FeCO e as outras representam os neurônios utilizados na coleta de dados. Os valores 27 e 58 representam as frequências de excitação mecânica. O valor de 200 representa onde está localizado o canal em que foi gravado o sinal elétrico de $200 \mathrm{~Hz}$. 
ID-531 2724 X FETi 27200 ID-536 2724 X FETi 27200 ID-537 2724 X FETi 27200 ID-538 2724 X FETi 27200 ID-53927 24 X FETi 27200 ID-541 5824 X FETi 58200 ID-542 2724 X FETi 27200 ID-543 2724 X FETi 27200 ID-544 2724 X FETi 27200 ID-545 2724 X FETi 27200 ID-546 5824 X FETi 58200 ID-547 5824 X FETi 58200 ID-54927 24 X FETi 27200 ID-601 5824 X FETi 58200 ID-602 2724 X FETi 27200 ID-603 5824 X FETi 58200 ID-604 5824 X FETi 58200 ID-605 5824 X FETi 58200 ID-607 2724 X FETi 27200 ID-609 2724 X FETi 27200 ID-610 5824 X FETi 58200 ID-611 5824 X FETi 58200 ID-612 2724 X FETi 27200 ID-613 5824 X FETi 58200 ID-614 5824 X FETi 58200 ID-615 5824 X FETi 58200 ID-620 2724 X FETi 27200 ID-621 2724 X FETi 27200 ID-622 2724 X FETi 27200 ID-624 5824 X FETi 58200 ID-625 5824 X FETi 58200 ID-626 2724 X FETi 27200 ID-630 5824 X FETi 58200 ID-631 5824 X FETi 58200 ID-634 2724 X FETi 27200 ID-635 5824 X FETi 58200 ID-636 5824 X FETi 58200 ID-638 5824 X FETi 58200 ID-63958 24 X FETi 58200 ID-640 2724 X FETi 27200 ID-641 2724 X FETi 27200
ID-644 2724 X FETi 27200 ID-645 5824 X FETi 58200 ID-646 2724 X FETi 27200 ID-647 2724 X FETi 27200 ID-648 5824 X FETi 58200 ID-649 5824 X FETi 58200 ID-701 2724 X FCO 27200 ID-702 2724 X FCO 27200 ID-705 27 24 X FCO 27200 ID-706 2724 X FCO 27200 ID-707 27 24 X FCO 27200 ID-708 2724 X FCO 27200 ID-709 27 24 X FCO 27200 ID-711 2724 X FCO 27200 ID-712 2724 X FCO 27200 ID-714 2724 X FCO 27200 ID-715 5824 X FCO 27200 ID-716 2724 X FCO 27200 ID-717 27 24 X FCO 27200 ID-718 2724 X FCO 27200 ID-719 5824 X FCO 27200 ID-721 2724 X FCO 27200 ID-722 2724 X FCO 27200 ID-723 2724 X FCO 27200 ID-726 27 24 X FCO 27200 ID-727 5824 X FCO 27200 ID-728 27 24 X FCO 27200 ID-729 5824 X FCO 27200 ID-730 5824 X FCO 27200 ID-731 5824 X FCO 27200 ID-732 27 24 X FCO 27200 ID-733 2724 X FCO 27200 ID-734 5824 X FCO 27200 ID-736 5824 X FCO 27200 ID-737 27 24 X FCO 27200 ID-738 2724 X FCO 27200 ID-73958 24 X FCO 27200 ID-740 2724 X FCO 27200 ID-741 5824 X FCO 27200 ID-743 2724 X FCO 27200 ID-744 58 24 X FCO 27200 
ID-745 27 24 X FCO 27200 ID-747 5724 X FCO 27200 ID-748 27 24 X FCO 27200 ID-749 5824 X FCO 27200 ID-750 27 24 X FCO 27200 ID-751 5824 X FCO 27200 ID-752 27 24 X FCO 27200 ID-753 5824 X FCO 27200 ID-754 27 24 X FCO 27200 ID-755 5824 X FCO 27200 ID-759 5824 X FCO 27200 ID-760 2724 X FCO 27200 ID-761 5824 X FCO 27200 ID-762 X $24 \times$ FCO 27200 ID-763 X $24 \times$ FCO 27200 ID-764 5824 X FCO 27200 ID-765 58 24 X FCO 27200 ID-766 5824 X FCO 27200 ID-767 58 24 X FCO 27200 ID-768 27 24 X FCO 27200 ID-769 27 24 X FCO 27200 ID-770 5824 X FCO 27200 ID-771 27 24 X FCO 27200 ID-772 5824 X FCO 27200 ID-773 27 24 X FCO 27200 ID-774 27 24 X FCO 27200 ID-778 27 24 X FCO 27200 ID-779 5824 X FCO 27200 ID-780 58 24 X FCO 27200 ID-781 2724 X FCO 27200 ID-782 5824 X FCO 27200 ID-783 2724 X FCO 27200 ID-785 27 24 X FCO 27200 ID-903 2724 PFFlTi PSFlTi PIFlTi 200 ID-904 2724 PFFlTi PSFlTi PIFlTi 200 ID-905 2724 PFFlTi PSFlTi PIFlTi 200 ID-906 2724 PFFlTi PSFlTi PIFlTi 200 ID-907 2724 PFFlTi PSFlTi PIFlTi 200 ID-908 2724 PFFlTi PSFlTi PIFlTi 200 ID-912 2724 PFFlTi PSFlTi PIFlTi 200 ID-913 5824 PFFlTi PSFlTi PIFlTi 200
ID-914 2724 PFFlTi PIFlTi PSFlTi 200 ID-915 5824 PFFlTi PIFlTi PSFlTi 200 ID-916 5824 PFFlTi PIFlTi PSFlTi 200 ID-917 5824 PFFlTi PIFlTi PSFlTi 200 ID-918 2724 PFFlTi PIFlTi PSFlTi 200 ID-919 2710 nonspiker PSFITi X 200 ID-920 5810 nonspiker PSFITi X 200 ID-921 $58 \quad 10$ FCO X 58200 ID-922 $27 \quad 10$ FCO X 27200 ID-923 $27 \quad 10$ FCO X 27200 ID-924 $58 \quad 10$ FCO X 58200 ID-925 10024 FCO X 100200 ID-926 $27 \quad 10 \quad$ FCO X 27200 ID-927 $58 \quad 10$ FCO X 58200 ID-928 10010 FCO X 100200 ID-929 $27 \quad 10$ FCO X 27200 ID-930 $58 \quad 10 \quad$ FCO X 58200 ID-931 10024 FCO X 100200 ID-932 $27 \quad 10 \quad$ FCO X 27200 ID-933 $58 \quad 10$ FCO X 58200 ID-934 $27 \quad 10$ FCO X 27200 ID-935 $58 \quad 10$ FCO X 58200 ID-936 10024 FCO X 100200 ID-937 $27 \quad 10$ FCO X 27200 ID-938 $58 \quad 10 \quad$ FCO X $58 \quad 200$ ID-939 $27 \quad 10 \quad$ FCO X $27 \quad 200$ ID-940 $58 \quad 10 \quad$ FCO X 58200 ID-942 $27 \quad 10$ FCO X 27200 ID-943 $58 \quad 10 \quad$ FCO X 58200 ID-944 10024 FCO X 100200 ID-945 $27 \quad 10$ FCO X 27200 ID-946 $58 \quad 10$ FCO X 58200 ID-947 10024 FCO X 100200 ID-948 $27 \quad 10 \quad$ FCO X $27 \quad 200$ ID-949 $58 \quad 10 \quad$ FCO X 58200 ID-958 10024 FCO X 100200 ID-1001 27 24 FCO X 27200 ID-1002 5824 FCO X 58200 ID-1003 10010 FCO X 100200 ID-1004 27 24 FCO X 27200 ID-1005 5824 FCO X 58200 
ID-1006 27 24 FCO X 27200

ID-1007 5824 FCO X 58200

ID-1008 2724 FCO X 27200

ID-1009 5824 FCO X 58200

ID-1010 27 24 FCO X 27200

ID-1011 5824 FCO X 58200

ID-1012 10010 FCO X 100200

ID-1013 27 24 FCO X 27200

ID-1014 5824 FCO X 58200

ID-1015 10010 FCO X 100200

ID-1016 27 24 FCO X 27200

ID-1017 5824 FCO X 58200

ID-1018 10010 FCO X 100200

ID-1019 27 24 FCO X 27200

ID-1020 5824 FCO X 58200

ID-1029 2710 PFFlTi PIFlTi X 27

ID-1030 5810 PFFlTi PIFlTi X 58

ID-1031 10010 PFFlTi PIFlTi PSFlTi 100

ID-1035 2710 PSFlTi PIFlTi X 200

ID-1036 2710 PSFITi PIFlTi X 200

ID-1037 5810 PSFlTi PIFlTi X 200

ID-1038 2724 Nonspiker PiFlTi X 200

ID-1039 5824 Nonspiker PiFlTi X 200

ID-1040 X 10 Nonspiker PIFlTi X X

ID-1201 2724 Nonspiker PIFlTi 27200

ID-1202 5824 Nonspiker PIFlTi 58200

ID-12065824 X PIFlTi 58200

ID-1207 2724 X PIFlTi 27200

ID-1208 X 24 Nonspiker PFFLTi X 200

ID-1209 2724 FETi SETi 27200

ID-1210 5824 FETi SETi 58200

ID-1211 2724 Nonspiker PiFlTi 27200

ID-1212 5824 Nonspiker PIFlTi 58200

ID-1213 5824 FCO X 58200

ID-1214 27 24 FCO X 27200

ID-1215 27 24 FCO X 27200

ID-1216 5824 FCO X 58200

ID-1217 10024 FCO X 100200

ID-1221 5824 FCO X 58200

ID-1222 2724 FCO X 27200

ID-122310024 FCO X 100200
ID-1224 5824 FCO X 58200

ID-1225 2724 FCO X 27200

ID-1226 10024 FCO X 100200

ID-1227 27 24 FCO X 27200

ID-1228 27 24 FCO X 27200

ID-1230 2724 FCO X 27200

ID-1231 5824 FCO X 58200

ID-1301 2710 PSFITi AFIFITi 27200

ID-1302 5810 PSFlTi AFIFlTi 58200

ID-1303 5810 nonspiker AFIFlTi 58200

ID-1304 2710 nonspiker AFIFlTi 27200

ID-1306 2710 nonspiker AFIFlTi 27200

ID-1307 5810 nonspiker AFIFlTi 58200

ID-1308 5810 nonspiker AFIFlTi 58200

ID-1309 2710 nonspiker AFIFlTi 27200

ID-1311 5810 nonspiker AFIFlTi 58200

ID-1312 2710 nonspiker AFIFlTi 27200

ID-1317 5810 flexor AFFlTi 58200

ID-1321 2724 nonspiker PlFlTi $27 \mathrm{X}$

ID-1322 5810 nonspiker PlFlTi $58 \mathrm{X}$

ID-1324 5810 nonspiker PlFlTi 58200

ID-1325 2710 FETi SETi 27200

ID-1326 5810 FETi SETi 58200

ID-1327 5810 FETi SETi 58200

ID-1328 $27 \quad 10$ FETi SETi 27200

ID-1329 $27 \quad 10$ ASFlTi X 27200

ID-1330 5810 ASFlTi X 58200

ID-1332 5810 PIFlTi X 58200

ID-1333 2710 PIFlTi X 27200

ID-1334 2710 FETi SETi 27200

ID-1335 5810 FETi SETi 58200

ID-1336 2710 PFFlTi PIFlTi 27200

ID-1337 5810 PFFlTi PIFlTi 58200

ID-1338 5810 Nonspiker PIFlTi 58200

ID-1339 2710 Nonspiker PIFlTi 27200

ID-1341 2710 PSFITi SETi 27200

ID-1342 5810 PSFlTi SETi 58200

ID-1343 5810 PSFlTi SETi 58200

ID-1344 2710 Nonspiker ASFlTi 27200

ID-1345 5810 Nonspiker ASFlTi 58200

ID-1346 5810 Nonspiker ASFlTi 58200 
ID-1347 2710 Nonspiker ASFlTi 27200 ID-1348 2710 Nonspiker PIFlTi 27200 ID-1349 5810 Nonspiker PIFlTi 58200 ID-1401 2710 Nonspiker PIFlTi 27200 ID-1402 5810 Nonspiker PlFlTi 58200 ID-1404 5810 Nonspiker Flexor SETi 58 ID-1405 2710 Nonspiker Flexor SETi 200 ID-1406 2710 FETi SETi 27200 ID-1407 5810 FETi SETi 58200 ID-1410 2710 Nonspiker AFFlTi SETi 200 ID-1411 5810 Nonspiker AFFlTi SETi 200 ID-1412 2710 Nonspiker AFFlTi SETi 200 ID-141327 10 Nonspiker AFFlTi SETi 200 ID-1414 5810 Nonspiker AFFlTi SETi 200 ID-1415 2710 Flexor SETi PSFITi 200 ID-1416 5824 Flexor SETi PSFlTi 58 ID-141727 10 Flexor SETi PSFITi 200 ID-1418 5824 Flexor SETi PSFlTi 58 ID-1419 2710 Nonspiker SETi PIFlTi 200 ID-1420 2710 Nonspiker SETi PIFlTi 200 ID-1421 5810 Nonspiker SETi PIFlTi 200 ID-1422 2710 Nonspiker SETi PIFlTi 200 ID-1423 5810 Nonspiker SETi PIFlTi 200 ID-1424 2710 Nonspiker PIFlTi PFFlTi 200 ID-1425 2710 Nonspiker PIFlTi PFFlTi 200 ID-1426 5810 Nonspiker PIFlTi PFFlTi 200 ID-1427 2710 FETi PIFlTi PFFlTi 200 ID-1428 5824 FETi PIFlTi PFFlTi 58 ID-1429 2710 Flexor PIFlTi PFFlTi 200 ID-1430 5824 Flexor PIFlTi PFFlTi 58
ID-1431 2710 Nonspiker SETi PFFlTi 200 ID-1432 5810 Nonspiker SETi PFFlTi 200 ID-1433 2710 Nonspiker SETi PFFlTi 200 ID-1434 2710 Nonspiker FLTi PSFlTi 200 ID-1435 2710 Nonspiker AfITi PSFITi 200 ID-1436 2710 Nonspiker AflTi PSFlTi 200 ID-1437 5824 Nonspiker AFlTi PSFITi 58 ID-1438 2710 ASFlTi PFFlTi PIFlTi 200 ID-1439 5824 ASFlTi PFFlTi PIFlTi 58 ID-1441 2710 Nonspiker SETi PiFlTi 200 ID-1442 2724 Nonspiker SETi $27 \mathrm{X}$ ID-144327 24 Nonspiker SETi $27 \mathrm{X}$ ID-1444 2724 Nonspiker SETi $27 \mathrm{X}$ ID-1445 5810 Nonspiker SETi 58 X ID-1501 2710 Nonspiker SETi PSFlTi 200 ID-1502 5824 Nonspiker SETi PSFlTi 58 ID-1503 2710 2000nOff SETi PSFlTi 200 ID-1504 2710 2000nOff SETi PSFlTi 200 ID-1505 2710 Nonspiker SETi PIFlTi 200 ID-1506 5810 Nonspiker SETi PIFlTi 200 ID-1507 2710 Nonspiker SETi PIFlTi 200 ID-1508 2710 Nonspiker SETi PIFlTi 200 ID-1509 5810 Nonspiker SETi PIFlTi 200 ID-1510 2710 Flexors SETi PIFlTi 200 ID-1512 2710 Nonspiker SETi PIFlTi 200 ID-1513 5810 Nonspiker SETi PIFlTi 200 ID-1514 2724 Flexor SETi PIFlTi 200 ID-1516 2710 Nonspiker SETi PSFlTi 200 